\title{
Substituted Dibenzodiazocines: Rapid Synthesis and Photochemical Properties
}

Felix Klockmann, ${ }^{1}$ Camilla Fangmann, ${ }^{1}$ Elena Zender, ${ }^{1}$ Tobias Schanz, ${ }^{1}$ Claudia Catapano, ${ }^{1}$ Andreas Terfort ${ }^{1, *}$

${ }^{1}$ Institute of Inorganic and Analytical Chemistry, University of Frankfurt, Max-von-LaueStrasse 7, 60438 Frankfurt, Germany)

E-mail: aterfort@chemie.uni-frankfurt.de

\section{Table of contents}

1. Experimental Details, Purification, Yields and Spectroscopic Data S2

1.1 Diphenylethanes 2a-n

1.2 Dibenzodiazocines 1a-n

2. UV-Vis spectra and kinetics

S14

2.1 UV-Vis spectra of substituted dibenzodiazocines 1c-n

2.2 Determination of thermal half-life of the $E$-isomers

3. NMR spectra of synthesized compounds 


\section{Experimental Details, Purification, Yields and Spectroscopic Data}

\subsection{Diphenylethanes 2a-n}

\section{1,2-Bis(2-nitrophenyl)ethane 2a}

2-Nitrotoluene (2.73 g, $191.9 \mathrm{mmol}), \mathrm{t}-\mathrm{BuOK}$ in THF (1.60 M, $13 \mathrm{~mL}, 21 \mathrm{mmol})$ and bromine $(1.0 \mathrm{~mL}, 20 \mathrm{mmol})$ were reacted.

${ }^{1} \mathrm{H}$ NMR (400 MHz, $\left.\mathrm{CDCl}_{3}\right): \delta 7.96(\mathrm{~d}, J=8.1 \mathrm{~Hz}, 2 \mathrm{H}), 7.54(\mathrm{t}, J=7.5 \mathrm{~Hz}, 2 \mathrm{H}), 7.40(\mathrm{~m}, 4 \mathrm{H})$, $3.25(\mathrm{~s}, 4 \mathrm{H})$.

Purification: excess 2-nitrotoluene was removed in vacuum at $80{ }^{\circ} \mathrm{C}$. The residue was dissolved in DCM and filtered through a pad of silica gel.

Appearance: yellow solid

Yield: $\quad 1.63 \mathrm{~g}, 60 \%$

\section{1,2-Bis(2-fluoro-6-nitrophenyl)ethane $2 \mathrm{~b}$}

2-Fluoro-6-nitrotoluene (3.00 g, $19.3 \mathrm{mmol})$, t-BuOK in THF (1.60 M, $24 \mathrm{~mL}, 38 \mathrm{mmol}$ ) and bromine (1.0 mL, $20 \mathrm{mmol})$ were employed.

${ }^{1} \mathrm{H}$ NMR (400 MHz, $\left.\mathrm{CDCl}_{3}\right): \delta 7.70(\mathrm{~d}, J=8.1 \mathrm{~Hz}, 2 \mathrm{H}), 7.36(\mathrm{td}, J=8.2,5.4 \mathrm{~Hz}, 2 \mathrm{H}), 7.27(\mathrm{t}$, $J=8.1 \mathrm{~Hz}, 2 \mathrm{H}), 3.32(\mathrm{~s}, 4 \mathrm{H})$.

${ }^{13} \mathrm{C}$ NMR $\left(101 \mathrm{MHz}, \mathrm{CDCl}_{3}\right): \delta 161.6(\mathrm{~d}, J=249.6 \mathrm{~Hz}), 150.6(\mathrm{~d}, J=4.8 \mathrm{~Hz}), 128.4(\mathrm{~d}, J=9.7$ $\mathrm{Hz}), 123.7(\mathrm{~d}, J=20.3 \mathrm{~Hz}), 120.5(\mathrm{~d}, J=3.4 \mathrm{~Hz}), 120.1(\mathrm{~d}, J=24.1 \mathrm{~Hz}), 25.0(\mathrm{dd}, J=2.9,1.4$ $\mathrm{Hz})$.

ESI-MS: $\quad 308.97[\mathrm{M}+\mathrm{H}]^{+}$ $330.92[\mathrm{M}+\mathrm{Na}]^{+}$

HRMS: $\quad$ found $309.06812[\mathrm{M}+\mathrm{H}]^{+}$ calc. $\quad 309.06814$

Purification: column chromatography (DCM)

Appearance: yellow solid

Yield: $\quad 2.20 \mathrm{~g}, 74 \%$

\section{1,2-Bis(2-chloro-6-nitrophenyl)ethane 2c}

2-Chloro-6-nitrotoluene (5.20 g, $29.1 \mathrm{mmol})$, THF (55 mL), t-BuOK in THF (1.60 M, $36 \mathrm{~mL}$, $58 \mathrm{mmol})$ and bromine $(1.5 \mathrm{~mL}, 29 \mathrm{mmol})$ were used. 
${ }^{1} \mathrm{H}$ NMR $\left(400 \mathrm{MHz}, \mathrm{CDCl}_{3}\right): \delta 7.64(\mathrm{dd}, J=8.1,1.2 \mathrm{~Hz}, 2 \mathrm{H}), 7.60(\mathrm{dd}, J=8.1,1.2 \mathrm{~Hz}, 2 \mathrm{H})$, $7.32(\mathrm{t}, J=8.1 \mathrm{~Hz}, 2 \mathrm{H}), 3.39(\mathrm{~s}, 4 \mathrm{H})$.

${ }^{13} \mathrm{C} \mathrm{NMR}\left(101 \mathrm{MHz}, \mathrm{CDCl}_{3}\right): \delta 151.8,137.0,133.9,132.5,128.3,123.1,28.3$.

ESI-MS: $\quad 341.00[\mathrm{M}+\mathrm{H}]^{+}$

HRMS: $\quad$ found $340.00121[\mathrm{M}]^{+}$

calc. $\quad 340.00176$

Purification: filtered through a pad of silica gel with DCM as eluent

Appearance: orange solid

Yield: $\quad 3.97 \mathrm{~g}, 80 \%$

\section{1,2-Bis(2-bromo-6-nitrophenyl)ethane 2d}

2-Bromo-6-nitrotoluene (4.02 g, $18.6 \mathrm{mmol})$, t-BuOK in THF (1.60 M, $12.5 \mathrm{~mL}, 20.0 \mathrm{mmol})$ and bromine $(1.0 \mathrm{~mL}, 19 \mathrm{mmol})$ were reacted.

${ }^{1} \mathrm{H}$ NMR $\left(400 \mathrm{MHz}, \mathrm{CDCl}_{3}\right): \delta 7.78(\mathrm{~d}, J=8.0 \mathrm{~Hz}, 2 \mathrm{H}), 7.66(\mathrm{~d}, J=8.1 \mathrm{~Hz}, 2 \mathrm{H}), 7.24(\mathrm{t}, J=$ $8.1 \mathrm{~Hz}, 2 \mathrm{H}), 3.43$ (s, 4H).

${ }^{13} \mathrm{C}$ NMR (101 MHz, $\left.\mathrm{CDCl}_{3}\right): \delta 151.7,137.3,133.8,128.6,127.4,123.8,30.8$.

ESI-MS: $\quad 431.16[\mathrm{M}+\mathrm{H}]^{+}$

HRMS: $\quad$ found $430.93768[\mathrm{M}+\mathrm{H}]^{+}$

calc. $\quad 430.90596$

found $452.88742[\mathrm{M}+\mathrm{Na}]^{+}$

calc. $\quad 452.88791$

Remarks: The synthesis was performed under exclusion of light.

Purification: $\quad$ column chromatography $($ cyclohexane/EtOAc $=5 / 1)$

Appearance: yellow solid

Yield: $\quad 2.54 \mathrm{~g}, 64 \%$

\section{1,2-Bis(6-trifluoromethyl-2-nitrophenyl)ethane $2 \mathrm{e}$}

6-Trifluoromethyl-2-nitrotoluene (4.05 g, $19.7 \mathrm{mmol})$, t-BuOK in THF (1.60 M, $13 \mathrm{~mL}, 21$ $\mathrm{mmol})$ and bromine $(1.0 \mathrm{~mL}, 20 \mathrm{mmol})$ reacted under the conditions described above.

${ }^{1} \mathrm{H}$ NMR (400 MHz, $\left.\mathrm{CDCl}_{3}\right): \delta 7.90(\mathrm{~d}, J=8.0 \mathrm{~Hz}, 2 \mathrm{H}), 7.86(\mathrm{~d}, J=8.1 \mathrm{~Hz}, 2 \mathrm{H}), 7.53(\mathrm{t}, J=$ $8.0 \mathrm{~Hz}, 2 \mathrm{H}), 3.43(\mathrm{~s}, 4 \mathrm{H})$.

${ }^{13} \mathrm{C} \mathrm{NMR}\left(101 \mathrm{MHz}, \mathrm{CDCl}_{3}\right): \delta 152.3(\mathrm{~s}), 133.1$ (s), 131.6 (q, $\left.J=30.6 \mathrm{~Hz}\right), 130.3(\mathrm{~s}), 128.3$ (s), 128.1 (s), 123.4 (q, $J=274.6 \mathrm{~Hz}), 27.8(\mathrm{~s})$.

ESI-MS: $\quad 339.12\left[{\left.\mathrm{M}-\mathrm{CF}_{3}\right]^{+}}^{+}\right.$ 


$$
431.15[\mathrm{M}+\mathrm{Na}]^{+}
$$

HRMS: $\quad$ found $431.04974[\mathrm{M}+\mathrm{Na}]^{+}$

calc. 431.04370

found $447.01737[\mathrm{M}+\mathrm{K}]^{+}$

calc. $\quad 447.01763$

Purification: column chromatography $($ cyclohexane/EtOAc $=3 / 1)$

Appearance: orange solid

Yield: $\quad 3.18 \mathrm{~g}, 79 \%$

\section{1,2-Bis(2-dimethylamino-6-nitrophenyl)ethane $2 f$}

3f $(1.83 \mathrm{~g}, 10.2 \mathrm{mmol})$ was reacted with t-BuOK in THF (1.60 M, $13 \mathrm{~mL}, 20 \mathrm{mmol})$ and bromine $(0.52 \mathrm{~mL}, 10 \mathrm{mmol})$.

${ }^{1} \mathrm{H}$ NMR (400 MHz, $\mathrm{CDCl}_{3}$ ): $\delta 7.47$ - $7.38(\mathrm{~m}, 2 \mathrm{H}), 7.22-7.15(\mathrm{~m}, 4 \mathrm{H}), 3.25(\mathrm{~s}, 4 \mathrm{H}), 2.50(\mathrm{~s}$, $12 \mathrm{H})$.

${ }^{13} \mathrm{C}$ NMR (101 MHz, $\left.\mathrm{CDCl}_{3}\right): \delta 155.4,151.9,131.3,127.1,124.5,119.4,45.2,26.3$.

ESI-MS: $\quad 359.04[\mathrm{M}+\mathrm{H}]^{+}$

HRMS: $\quad$ found $358.16356[\mathrm{M}]^{+}$

calc. $\quad 358.16411$

Purification: column chromatography $(n$-hexane/EtOAc $=4 / 1)$

Appearance: yellow solid

Yield: $\quad 1.19 \mathrm{~g}, 65 \%$

\section{1,2-Bis(5-methoxy-2-nitrophenyl)ethane $2 \mathrm{~g}$}

5-Methoxy-2-nitrotoluene (4.98 g, $29.8 \mathrm{mmol})$, t-BuOK in THF (1.60 M, $37 \mathrm{~mL}, 60 \mathrm{mmol})$ and bromine $(1.5 \mathrm{~mL}, 30 \mathrm{mmol})$ were employed.

${ }^{1} \mathrm{H} \mathrm{NMR}\left(400 \mathrm{MHz}, \mathrm{CDCl}_{3}\right): \delta 8.09(\mathrm{~d}, \mathrm{~J}=9.1 \mathrm{~Hz}, 2 \mathrm{H}), 6.89(\mathrm{~d}, \mathrm{~J}=2.6 \mathrm{~Hz}, 2 \mathrm{H}), 6.84(\mathrm{dd}, \mathrm{J}=$ 9.1, 2.6 Hz, 2H), 3.89 (s, 6H), 3.29 (s, 4H).

${ }^{13} \mathrm{C} \mathrm{NMR}\left(126 \mathrm{MHz}, \mathrm{CDCl}_{3}\right): \delta 163.5,142.1,139.8,127.9,116.8,113.2,56.0,35.4$.

ESI-MS: $\quad 355.05[\mathrm{M}+\mathrm{Na}]^{+}$

$330.98[\mathrm{M}-\mathrm{H}]^{-}$

HRMS: $\quad$ found $355.09015[\mathrm{M}+\mathrm{Na}]^{+}$

calc. $\quad 355.09006$

HRMS: $\quad$ found $371.06409[\mathrm{M}+\mathrm{K}]^{+}$

calc. $\quad 371.06399$ 
Purification: column chromatography $(n$-hexane/EtOAc $=1 / 1)$

Appearance: beige solid

Yield: $\quad 693 \mathrm{~g}, 14 \%$

\section{1,2-Bis(5-(methoxymethoxy)-2-nitrophenyl)ethane $2 \mathrm{~h}$}

3h (4.98 g, $25.4 \mathrm{mmol})$, THF (60 mL), t-BuOK in THF (1.60 M, $32 \mathrm{~mL}, 51 \mathrm{mmol})$ and bromine (1.3 mL, $25 \mathrm{mmol})$ were used.

${ }^{1} \mathrm{H}$ NMR (400 MHz, $\left.\mathrm{CDCl}_{3}\right): \delta 8.05(\mathrm{~d}, J=9.9 \mathrm{~Hz}, 2 \mathrm{H}), 7.04-6.88(\mathrm{~m}, 4 \mathrm{H}), 5.22(\mathrm{~s}, 4 \mathrm{H}), 3.48$ (s, 6H), $3.28(\mathrm{~s}, 4 \mathrm{H})$.

${ }^{13} \mathrm{C} \mathrm{NMR}\left(101 \mathrm{MHz}, \mathrm{CDCl}_{3}\right): \delta 161.0,143.0,139.4,127.8,119.4,114.5,94.4,56.6,35.0$.

ESI-MS: $\quad 414.89[\mathrm{M}+\mathrm{Na}]^{+}$

HRMS: $\quad$ found $415.11089[\mathrm{M}+\mathrm{Na}]^{+}$

calc. $\quad 415.11119$

Purification: gradient sublimation $\left(185^{\circ} \mathrm{C}, 10^{-5} \mathrm{mbar}, 5 \mathrm{~h}\right)$

Appearance: beige solid

Yield: $\quad 1.05 \mathrm{~g}, 21 \%$

\section{1,2-Bis(5-benzyloxy-2-nitrophenyl)ethane $2 \mathrm{i}$}

5-Benzyloxy-2-nitrotoluene (1.51 g, $6.17 \mathrm{mmol})$, t-BuOK in THF (1.60 M, $7.7 \mathrm{~mL}, 12 \mathrm{mmol})$ and bromine $(0.3 \mathrm{~mL}, 6 \mathrm{mmol})$ were used.

${ }^{1} \mathrm{H}$ NMR (400 MHz, $\left.\mathrm{CDCl}_{3}\right): \delta 8.08(\mathrm{~d}, J=9.1 \mathrm{~Hz}, 2 \mathrm{H}), 7.51-7.32(\mathrm{~m}, 10 \mathrm{H}), 6.99(\mathrm{~d}, J=2.7$ $\mathrm{Hz}, 2 \mathrm{H}), 6.91$ (dd, $J=9.1,2.7 \mathrm{~Hz}, 2 \mathrm{H}), 5.14$ (s, 4H), 3.27 (s, 4H).

${ }^{13} \mathrm{C} \mathrm{NMR}\left(101 \mathrm{MHz}, \mathrm{CDCl}_{3}\right): \delta 162.6,142.2,139.8,135.7,128.9,128.6,127.9,127.8,117.8$, $113.9,70.7,35.4$.

ESI-MS: $\quad 507.19[\mathrm{M}+\mathrm{Na}]^{+}$

HRMS: $\quad$ found $507.15042[\mathrm{M}+\mathrm{Na}]^{+}$

calc. $\quad 507.15266$

Purification: gradient sublimation $\left(130{ }^{\circ} \mathrm{C}, 10^{-5} \mathrm{mbar}, 2.5 \mathrm{~h}\right)$

Appearance: brownish solid

Yield: $\quad 852 \mathrm{mg}, 57 \%$

\section{1,2-Bis(3-fluoro-6-nitrophenyl)ethane $2 \mathrm{j}$}

5-Fluor-2-nitrotoluene $(2.00 \mathrm{~g}, 12.9 \mathrm{mmol})$ was reacted with t-BuOK in THF (1.60 M, 8.5 mL, $14 \mathrm{mmol})$ and bromine $(0.7 \mathrm{~mL}, 14 \mathrm{mmol})$. 
${ }^{1} \mathrm{H}$ NMR $\left(400 \mathrm{MHz}, \mathrm{CDCl}_{3}\right): \delta 8.08(\mathrm{dd}, J=9.0,5.1 \mathrm{~Hz}, 2 \mathrm{H}), 7.14(\mathrm{dd}, J=8.8,2.7 \mathrm{~Hz}, 2 \mathrm{H})$, $7.12-7.05$ (m, 2H), 3.28 (s, 4H).

${ }^{13} \mathrm{C} \mathrm{NMR}\left(101 \mathrm{MHz}, \mathrm{CDCl}_{3}\right): \delta 165.0(\mathrm{~d}, J=257.6 \mathrm{~Hz}), 145.3(\mathrm{~s}), 139.6(\mathrm{~d}, J=9.0 \mathrm{~Hz}), 128.1$ $(\mathrm{d}, J=10.0 \mathrm{~Hz}), 119.2(\mathrm{~d}, J=23.1 \mathrm{~Hz}), 115.1(\mathrm{~d}, J=23.2 \mathrm{~Hz}), 34.6(\mathrm{~s})$.

HRMS: $\quad$ found $309.06767[\mathrm{M}+\mathrm{H}]^{+}$ calc. $\quad 309.06814$

Purification: column chromatography $($ cyclohexane/EtOAc $=5 / 1)$

Appearance: yellow solid

Yield: $\quad 1.41 \mathrm{~g}, 71 \%$

\section{1,2-Bis(4-methoxy-2-nitrophenyl)ethane $2 \mathrm{k}$}

4-Methoxy-2-nitrotoluene (0.36 g, $2.2 \mathrm{mmol})$, t-BuOK in THF (1.60 M, $2.7 \mathrm{~mL}, 4.3 \mathrm{mmol})$ and bromine $(0.11 \mathrm{~mL}, 2.2 \mathrm{mmol})$ were used.

${ }^{1} \mathrm{H}$ NMR (400 MHz, $\left.\mathrm{CDCl}_{3}\right): \delta 7.46(\mathrm{~d}, J=2.7 \mathrm{~Hz}, 2 \mathrm{H}), 7.28(\mathrm{~d}, J=8.5 \mathrm{~Hz}, 2 \mathrm{H}), 7.08(\mathrm{dd}, J=$ 8.5, $2.7 \mathrm{~Hz}, 2 \mathrm{H}), 3.86(\mathrm{~s}, 6 \mathrm{H}), 3.14(\mathrm{~s}, 4 \mathrm{H})$.

${ }^{13} \mathrm{C} \mathrm{NMR}\left(101 \mathrm{MHz}, \mathrm{CDCl}_{3}\right): \delta$ 158.6, 149.7, 133.5, 128.3, 120.3, 109.4, 56.0, 34.1.

ESI-MS: $\quad 354.87[\mathrm{M}+\mathrm{Na}]^{+}$

HRMS: $\quad$ found $332.10029[\mathrm{M}]^{+}$

calc. $\quad 332.10000$

Purification: column chromatography $(n$-hexane/EtOAc $=4 / 1)$

Appearance: yellow solid

Yield: $\quad 91 \mathrm{mg}, 25 \%$

\section{1,2-Bis(4-(methoxymethoxy)-2-nitrophenyl)ethane 21}

The compound was formed from 31 (2.50 g, $12.6 \mathrm{mmol})$, t-BuOK in THF (1.60 M, $16 \mathrm{~mL}, 25$ $\mathrm{mmol})$ and bromine $(0.70 \mathrm{~mL}, 13 \mathrm{mmol})$.

${ }^{1} \mathrm{H}$ NMR (400 MHz, $\left.\mathrm{CDCl}_{3}\right): \delta 7.63(\mathrm{~d}, J=2.6 \mathrm{~Hz}, 2 \mathrm{H}), 7.32(\mathrm{~d}, J=8.5 \mathrm{~Hz}, 2 \mathrm{H}), 7.22(\mathrm{dd}, J=$ 8.5, 2.6 Hz, 2H), $5.21(\mathrm{~s}, 4 \mathrm{H}), 3.49$ (s, 6H), $3.13(\mathrm{~s}, 4 \mathrm{H})$.

${ }^{13} \mathrm{C} \mathrm{NMR}\left(101 \mathrm{MHz}, \mathrm{CDCl}_{3}\right): \delta 156.2,149.6,133.4,129.3,121.8,112.4,94.8,56.4,34.1$.

ESI-MS: $\quad 414.99[\mathrm{M}+\mathrm{Na}]^{+}$

HRMS: $\quad$ found $415.11078[\mathrm{M}+\mathrm{Na}]^{+}$

calc. $\quad 415.11119$

Purification: gradient sublimation $\left(180{ }^{\circ} \mathrm{C}, 10^{-5}\right.$ mbar, $1.5 \mathrm{~h}$ )

Appearance: brown solid 
Yield:

$890 \mathrm{mg}, 36 \%$

\section{1,2-Bis(3-fluoro-2-nitrophenyl)ethane $2 \mathrm{~m}$}

3-Fluor-2-nitrotoluene (1.50 g, $9.65 \mathrm{mmol})$, t-BuOK in THF (1.60 M, $12 \mathrm{~mL}, 19 \mathrm{mmol})$ and bromine $(0.50 \mathrm{~mL}, 9.8 \mathrm{mmol})$ were employed.

${ }^{1} \mathrm{H}$ NMR (400 MHz, $\left.\mathrm{CDCl}_{3}\right): \delta 7.42(\mathrm{td}, J=8.1,5.3 \mathrm{~Hz}, 2 \mathrm{H}), 7.15(\mathrm{t}, J=8.9 \mathrm{~Hz}, 2 \mathrm{H}), 7.07$ (d, $J=7.8 \mathrm{~Hz}, 2 \mathrm{H}), 2.99(\mathrm{~s}, 4 \mathrm{H})$.

${ }^{13} \mathrm{C}$ NMR (101 MHz, $\left.\mathrm{CDCl}_{3}\right): \delta 154.1(\mathrm{~d}, J=258.1 \mathrm{~Hz}), 140.0(\mathrm{~d}, J=10.3 \mathrm{~Hz}), 135.1(\mathrm{~d}, J=$ $1.4 \mathrm{~Hz}), 132.4$ (d, $J=8.3 \mathrm{~Hz}), 126.4(\mathrm{~d}, J=3.6 \mathrm{~Hz}), 115.7$ (d, $J=19.2 \mathrm{~Hz}), 32.9$ (d, $J=1.8$ $\mathrm{Hz})$.

ESI-MS: $\quad 308.97[\mathrm{M}+\mathrm{H}]^{+}$ $330.95[\mathrm{M}+\mathrm{Na}]^{+}$

HRMS: $\quad$ found $309.06744[\mathrm{M}+\mathrm{H}]^{+}$

calc. $\quad 309.06814$

Purification: gradient sublimation $\left(125^{\circ} \mathrm{C}, 10^{-5} \mathrm{mbar}, 2 \mathrm{~h}\right)$

Appearance: yellow solid

Yield: $\quad 952 \mathrm{mg}, 64 \%$

\section{1,2-Bis(3-methyl-2-nitrophenyl)ethane 2n}

2-Nitro-m-xylene $(1.00 \mathrm{~g}, 6.62 \mathrm{mmol})$ was reacted with t-BuOK in THF (1.60 M, $4.3 \mathrm{~mL}, 7.0$ $\mathrm{mmol})$ and bromine $(0.30 \mathrm{~mL}, 6.6 \mathrm{mmol})$.

${ }^{1} \mathrm{H}$ NMR (400 MHz, $\left.\mathrm{CDCl}_{3}\right): \delta 7.29\left(\mathrm{t},{ }^{3} J=7.6 \mathrm{~Hz}, 2 \mathrm{H}\right), 7.16\left(\mathrm{~d},{ }^{3} J=7.7 \mathrm{~Hz}, 2 \mathrm{H}\right), 7.09\left(\mathrm{~d},{ }^{3} J\right.$ $=7.7 \mathrm{~Hz}, 2 \mathrm{H}), 2.85(\mathrm{~s}, 4 \mathrm{H}), 2.33(\mathrm{~s}, 6 \mathrm{H})$.

${ }^{13} \mathrm{C}-\mathrm{NMR}\left(101 \mathrm{MHz}, \mathrm{CDCl}_{3}\right): \delta$ 151.72, 132.28, 130.38, 129.81, 129.68, 128.53, 33.21, 17.61.

ESI-MS: $\quad 300.33[\mathrm{M}]^{+}$

HRMS: $\quad$ found $323.10006[\mathrm{M}+\mathrm{Na}]^{+}$

calc. $\quad 323.10023$

Purification: gradient sublimation $\left(110{ }^{\circ} \mathrm{C}, 10^{-5} \mathrm{mbar}, 15 \mathrm{~h}\right)$

Appearance: yellow solid

Yield: $\quad 437 \mathrm{mg}, 44 \%$ 


\subsection{Dibenzodiazocines 1a-n}

\section{1,12-Dihydrodibenzo[c,g]-1,2-diazocine 1a}

2a (404 mg, $1.48 \mathrm{mmol}$ ) was reacted with $\mathrm{LiAlH}_{4}$ in THF (2.40 M, $12 \mathrm{~mL}, 29 \mathrm{mmol}$ ).

${ }^{1} \mathrm{H}$ NMR $\left(600 \mathrm{MHz}, \mathrm{CDCl}_{3}\right): \delta 7.13(\mathrm{t}, J=7.5 \mathrm{~Hz}, 2 \mathrm{H}), 7.01(\mathrm{t}, J=7.4 \mathrm{~Hz}, 2 \mathrm{H}), 6.97(\mathrm{~d}, J=$ $7.4 \mathrm{~Hz}, 2 \mathrm{H}), 6.82(\mathrm{~d}, J=7.8 \mathrm{~Hz}, 2 \mathrm{H}), 2.88(\mathrm{~m}, 4 \mathrm{H})$.

Purification: column chromatography $(n$-hexane/EtOAc $=4 / 1)$

Appearance: yellow solid

Yield: $\quad 179 \mathrm{mg}, 58 \%$

\section{1,10-Difluoro-11,12-dihydrodibenzo[c,g]-1,2-diazocine 1b}

2b (1.00 g, $3.24 \mathrm{mmol})$ and $\mathrm{LiAlH}_{4}$ in THF $(2.40 \mathrm{M}, 27 \mathrm{~mL}, 65 \mathrm{mmol})$ were reacted.

${ }^{1} \mathrm{H}$ NMR (400 MHz, $\left.\mathrm{CDCl}_{3}\right): \delta 7.12(\mathrm{td}, J=8.0,5.7 \mathrm{~Hz}, 2 \mathrm{H}), 6.81(\mathrm{t}, J=8.8 \mathrm{~Hz}, 2 \mathrm{H}), 6.66(\mathrm{~d}$, $J=7.9 \mathrm{~Hz}, 2 \mathrm{H}), 3.30-3.12(\mathrm{~m}, 2 \mathrm{H}), 2.74-2.56(\mathrm{~m}, 2 \mathrm{H})$.

${ }^{13} \mathrm{C} \mathrm{NMR}\left(101 \mathrm{MHz}, \mathrm{CDCl}_{3}\right): \delta 160.4(\mathrm{~d}, J=247.7 \mathrm{~Hz}), 156.7(\mathrm{~d}, J=3.1 \mathrm{~Hz}), 127.8(\mathrm{~d}, J=9.2$ Hz), 116.0 (d, $J=18.3 \mathrm{~Hz}), 114.8(\mathrm{~d}, J=3.7 \mathrm{~Hz}), 113.7$ (d, $J=23.4 \mathrm{~Hz}), 22.7$ (d, $J=3.6 \mathrm{~Hz})$.

ESI-MS: $\quad 245.07[\mathrm{M}+\mathrm{H}]^{+}$

HRMS: $\quad$ found $245.08896[\mathrm{M}+\mathrm{H}]^{+}$

calc. 245.08848

Purification: column chromatography $(n$-hexane/EtOAc $=3 / 1)$

Appearance: yellow solid

Yield: $\quad 301 \mathrm{mg}, 38 \%$

\section{1,10-Dichloro-11,12-dihydrodibenzo[c,g]-1,2-diazocine 1c}

2c (585 mg, $1.47 \mathrm{mmol})$ and $\mathrm{LiAlH}_{4}$ in THF (2.40 M, $\left.12.2 \mathrm{~mL}, 29.3 \mathrm{mmol}\right)$ were employed. ${ }^{1} \mathrm{H} \mathrm{NMR}\left(400 \mathrm{MHz}, \mathrm{CDCl}_{3}\right): \delta 7.14(\mathrm{dd}, J=8.0,1.2 \mathrm{~Hz}, 2 \mathrm{H}), 7.07$ (t, $\left.J=7.9 \mathrm{~Hz}, 2 \mathrm{H}\right), 6.74$ (dd, $J=7.8,1.2 \mathrm{~Hz}, 2 \mathrm{H}), 3.50-3.29$ (m, 2H), $2.86-2.69$ (m, 2H).

${ }^{13} \mathrm{C}$ NMR (101 MHz, $\left.\mathrm{CDCl}_{3}\right): \delta 156.4,134.7,128.2,127.5,126.1,117.5,27.5$.

HRMS: $\quad$ found $276.02156[\mathrm{M}]^{+}$

calc. 276.02210

Purification: column chromatography $(n$-hexane/EtOAc $=4 / 1)$

Appearance: yellow solid

Yield: $\quad 106 \mathrm{mg}, 26 \%$ 


\section{1,10-Dibromo-11,12-dihydrodibenzo[c,g]-1,2-diazocine 1d}

$\mathrm{LiAlH}(\mathrm{OMe})_{3}$ was first formed by reaction of $\mathrm{LiAlH}_{4}$ in THF $(2.40 \mathrm{M}, 10.7 \mathrm{~mL}, 25.6 \mathrm{mmol})$ with $\mathrm{MeOH}(3.12 \mathrm{~mL}, 76.8 \mathrm{mmol})$ at $0{ }^{\circ} \mathrm{C}$, before $2 \mathrm{~d}(550 \mathrm{mg}, 1.28 \mathrm{mmol})$ was added. The rest of the reaction proceeded like decribed above.

${ }^{1} \mathrm{H} \mathrm{NMR}\left(400 \mathrm{MHz}, \mathrm{CDCl}_{3}\right): \delta 7.33(\mathrm{~d}, J=8.0 \mathrm{~Hz}, 2 \mathrm{H}), 6.99(\mathrm{t}, J=7.9 \mathrm{~Hz}, 2 \mathrm{H}), 6.76(\mathrm{~d}, J=$ $7.8 \mathrm{~Hz}, 2 \mathrm{H}), 3.47-3.27$ (m, 2H), $2.88-2.71(\mathrm{~m}, 2 \mathrm{H})$.

${ }^{13} \mathrm{C} \mathrm{NMR}\left(101 \mathrm{MHz}, \mathrm{CDCl}_{3}\right): \delta 156.1,131.5,127.9,127.8,125.4,118.1,30.5$.

HRMS: $\quad$ found $366.92672[\mathrm{M}+\mathrm{H}]^{+}$

calc. $\quad 366.92630$

Remarks: $\quad \mathrm{LiAlH}(\mathrm{OMe})_{3}\left(20\right.$ eq. $\left.\mathrm{H}^{-}\right)$was used instead of $\mathrm{LiAlH}_{4}$. The reagent was prepared according to chapter 2.1 .

Purification: gradient sublimation $\left(125^{\circ} \mathrm{C}, 10^{-5}\right.$ mbar, $\left.1.5 \mathrm{~h}\right)$

Appearance: orange solid

Yield: $\quad 94 \mathrm{mg}, 20 \%$

\section{1,10-Bis(trifluoromethyl)-11,12-dihydrodibenzo[c,g]-1,2-diazocine 1e}

$2 \mathbf{e}(820 \mathrm{mg}, 2.00 \mathrm{mmol})$ and $\mathrm{LiAlH}_{4}$ in THF $(2.40 \mathrm{M}, 17 \mathrm{~mL}, 40 \mathrm{mmol})$ were employed.

${ }^{1} \mathrm{H} \mathrm{NMR}\left(400 \mathrm{MHz}, \mathrm{CDCl}_{3}\right): \delta 7.42(\mathrm{~d}, J=7.9 \mathrm{~Hz}, 2 \mathrm{H}), 7.24(\mathrm{t}, J=7.9 \mathrm{~Hz}, 2 \mathrm{H}), 6.96(\mathrm{~d}, J=$ $7.9 \mathrm{~Hz}, 2 \mathrm{H}), 3.37-3.21(\mathrm{~m}, 2 \mathrm{H}), 2.92-2.76(\mathrm{~m}, 2 \mathrm{H})$.

${ }^{13} \mathrm{C} \mathrm{NMR}\left(126 \mathrm{MHz}, \mathrm{CDCl}_{3}\right.$ ): $\delta 156.86$ (s), 130.24 - 129.20 (m), 126.96 (s), 126.44 (s), 125.06 - $124.88(\mathrm{~m}), 123.70$ (q, $J=274.7 \mathrm{~Hz}), 121.71(\mathrm{~s}), 26.58$ (s).

ESI-MS: $\quad 344.15[\mathrm{M}]^{+}$

HRMS: $\quad$ found $345.08215[\mathrm{M}+\mathrm{H}]^{+}$

calc. $\quad 345.08209$

Purification: gradient sublimation $\left(125^{\circ} \mathrm{C}, 10^{-5}\right.$ mbar, $\left.3.5 \mathrm{~h}\right)$

Appearance: yellow solid

Yield: $\quad 227 \mathrm{mg}, 33 \%$. This product contained some photo-inactive impurities, see the spectra in the Supp. Info.

\section{1,10-Bis(dimethylamino)-11,12-dihydrodibenzo[c,g]-1,2-diazocine 1f}

2f $(118 \mathrm{mg}, 0.279 \mathrm{mmol})$ and $\mathrm{LiAlH}_{4}$ in THF (2.40 M, $\left.2.3 \mathrm{~mL}, 5.6 \mathrm{mmol}\right)$ were used.

${ }^{1} \mathrm{H} \mathrm{NMR}\left(400 \mathrm{MHz}, \mathrm{CDCl}_{3}\right): \delta 7.02(\mathrm{t}, J=7.9 \mathrm{~Hz}, 2 \mathrm{H}), 6.75(\mathrm{dd}, J=8.0,0.9 \mathrm{~Hz}, 2 \mathrm{H}), 6.44(\mathrm{dd}$, $J=7.8,0.9 \mathrm{~Hz}, 2 \mathrm{H}), 3.53-3.34(\mathrm{~m}, 2 \mathrm{H}), 2.65-2.46(\mathrm{~m}, 2 \mathrm{H})$.

${ }^{13} \mathrm{C} \mathrm{NMR}\left(101 \mathrm{MHz}, \mathrm{CDCl}_{3}\right): \delta 157.7,153.1,126.8,123.8,117.1,112.7,45.0,24.9$. 
ESI-MS: $\quad 295.13[\mathrm{M}+\mathrm{H}]^{+}$

HRMS: $\quad$ found $295.19189[\mathrm{M}+\mathrm{H}]^{+}$

calc. 295.19172

Purification: column chromatography $(n$-hexane/EtOAc $=2 / 1)$

Appearance: yellow solid

Yield: $\quad 23 \mathrm{mg}, 28 \%$

\section{2,9-Dimethoxy-11,12-dihydrodibenzo[c,g]-1,2-diazocine $1 \mathrm{~g}$}

Obtained by reaction of $2 \mathrm{~g}(201 \mathrm{mg}, 0.602 \mathrm{mmol})$ with $\mathrm{LiAlH}_{4}$ in THF $(2.40 \mathrm{M}, 5.0 \mathrm{~mL}$, $12 \mathrm{mmol})$.

${ }^{1} \mathrm{H} \mathrm{NMR}\left(400 \mathrm{MHz}, \mathrm{CDCl}_{3}\right): \delta 6.81(\mathrm{~d}, J=8.6 \mathrm{~Hz}, 2 \mathrm{H}), 6.68(\mathrm{dd}, J=8.6,2.6 \mathrm{~Hz}, 2 \mathrm{H}), 6.52(\mathrm{~d}$, $J=2.6 \mathrm{~Hz}, 2 \mathrm{H}), 3.72(\mathrm{~s}, 6 \mathrm{H}), 2.83(\mathrm{~s}, 4 \mathrm{H})$.

${ }^{13} \mathrm{C}$ NMR (101 MHz, $\left.\mathrm{CDCl}_{3}\right): \delta 158.3,149.2,129.8,121.2,114.6,112.0,55.5,32.2$.

ESI-MS: $\quad 269.15[\mathrm{M}+\mathrm{H}]^{+}$

HRMS: found $269.12836[\mathrm{M}+\mathrm{H}]^{+}$

calc. $\quad 269.12845$

Purification: $\quad$ column chromatography $(n$-hexane/EtOAc $=1 / 1)$

Appearance: orange solid

Yield: $\quad 16 \mathrm{mg}, 10 \%$

\section{2,9-Bis(methoxymethoxy)-11,12-dihydrodibenzo[c,g]-1,2-diazocine $1 \mathrm{~h}$}

2h $(500 \mathrm{mg}, 1.27 \mathrm{mmol})$ and $\mathrm{LiAlH}_{4}$ in THF $(2.40 \mathrm{M}, 11 \mathrm{~mL}, 26 \mathrm{mmol})$ were used.

${ }^{1} \mathrm{H}$ NMR (600 MHz, CDCl $)$ : $\delta 6.87-6.77(\mathrm{~m}, 4 \mathrm{H}), 6.67(\mathrm{~d}, J=2.1 \mathrm{~Hz}, 2 \mathrm{H}), 5.08(\mathrm{~s}, 4 \mathrm{H}), 3.44$ (s, 6H), 2.83 (bs, 4H).

${ }^{13} \mathrm{C}$ NMR $\left(151 \mathrm{MHz}, \mathrm{CDCl}_{3}\right): \delta 156.1,150.1,129.7,121.2,117.0,114.6,94.7,56.2,32.1$.

ESI-MS: $\quad 329.20[\mathrm{M}+\mathrm{H}]^{+}$

HRMS: $\quad$ found $329.15016[\mathrm{M}+\mathrm{H}]^{+}$

calc. 329.14958

Purification: $\quad$ column chromatography $(n$-hexane/EtOAc $=2 / 1)$

Appearance: yellow solid

Yield: $\quad 92 \mathrm{mg}, 22 \%$

\section{2,9-Dibenzyloxy-11,12-dihydrodibenzo[c,g]-1,2-diazocine 1i}

$2 \mathbf{i}(310 \mathrm{mg}, 0.640 \mathrm{mmol})$ was reduced with $\mathrm{LiAlH}_{4}$ in THF (2.40 M, $\left.5.5 \mathrm{~mL}, 13 \mathrm{mmol}\right)$. 
${ }^{1} \mathrm{H}$ NMR $\left(400 \mathrm{MHz}, \mathrm{CDCl}_{3}\right): \delta 7.42-7.34(\mathrm{~m}, 10 \mathrm{H}), 6.82(\mathrm{~d}, J=8.6 \mathrm{~Hz}, 2 \mathrm{H}), 6.76(\mathrm{dd}, J=$ 8.6, 2.5 Hz, 2H), $6.62(\mathrm{~d}, J=2.5 \mathrm{~Hz}, 2 \mathrm{H}), 4.97$ (s, 4H), $2.82(\mathrm{bs}, 4 \mathrm{H})$.

${ }^{13} \mathrm{C} \mathrm{NMR}\left(101 \mathrm{MHz}, \mathrm{CDCl}_{3}\right): \delta 157.6,149.4,136.8,129.9,128.7,128.2,127.7,121.2,115.7$, $112.9,70.3,32.2$.

ESI-MS: $\quad 421.15[\mathrm{M}+\mathrm{H}]^{+}$

HRMS: $\quad$ found $421.19105[\mathrm{M}+\mathrm{H}]^{+}$

calc. 421.19099

Purification: column chromatography $(n$-hexane/EtOAc $=4 / 1)$

Appearance: yellow solid

Yield: $\quad 8 \mathrm{mg}, 3 \%$

\section{2,9-Difluoro-11,12-dihydrodibenzo[c,g]-1,2-diazocine $1 \mathbf{j}$}

2j (480 mg, $1.56 \mathrm{mmol})$ and $\mathrm{LiAlH}_{4}$ in THF (2.40 M, $\left.13 \mathrm{~mL}, 31 \mathrm{mmol}\right)$ were utilized.

${ }^{1} \mathrm{H}$ NMR $\left(400 \mathrm{MHz}, \mathrm{CDCl}_{3}\right): \delta 6.91-6.78(\mathrm{~m}, 4 \mathrm{H}), 6.72(\mathrm{dd}, J=9.2,2.4 \mathrm{~Hz}, 2 \mathrm{H}), 2.96(\mathrm{bs}$, $2 \mathrm{H}), 2.74$ (bs, 2H).

${ }^{13} \mathrm{C} \mathrm{NMR}\left(101 \mathrm{MHz}, \mathrm{CDCl}_{3}\right): \delta 161.2(\mathrm{~d}, J=246.6 \mathrm{~Hz}), 151.5(\mathrm{~d}, J=3.1 \mathrm{~Hz}), 130.3(\mathrm{~d}, J=7.8$ Hz), 121.0 (d, $J=8.8 \mathrm{~Hz}), 116.3$ (d, $J=22.4 \mathrm{~Hz}), 114.0$ (d, $J=22.6 \mathrm{~Hz}), 31.7$ (d, $J=1.3 \mathrm{~Hz})$.

HRMS: $\quad$ found $245.08874[\mathrm{M}+\mathrm{Na}]^{+}$ calc. $\quad 245.08848$

Purification: column chromatography $(n$-hexane/EtOAc $=4 / 1)$

Appearance: orange solid

Yield: $\quad 168 \mathrm{mg}, 44 \%$

\section{3,8-Dimethoxy-11,12-dihydrodibenzo[c,g]-1,2-diazocine $1 \mathrm{k}$}

2k (96.7 mg, $0.301 \mathrm{mmol})$ was reacted with $\mathrm{LiAlH}_{4}$ in THF (2.40 M, $\left.2.5 \mathrm{~mL}, 6.0 \mathrm{mmol}\right)$.

${ }^{1} \mathrm{H}$ NMR (400 MHz, $\left.\mathrm{CDCl}_{3}\right): \delta 6.87(\mathrm{~d}, J=8.4 \mathrm{~Hz}, 2 \mathrm{H}), 6.57(\mathrm{dd}, J=8.4,2.6 \mathrm{~Hz}, 2 \mathrm{H}), 6.38(\mathrm{~d}$, $J=2.6 \mathrm{~Hz}, 2 \mathrm{H}), 3.72(\mathrm{~s}, 6 \mathrm{H}), 2.95-2.58(\mathrm{~m}, 4 \mathrm{H})$.

${ }^{13} \mathrm{C} \mathrm{NMR}\left(101 \mathrm{MHz}, \mathrm{CDCl}_{3}\right): \delta 158.3,156.2,130.8,120.5,113.1,104.2,55.5,31.2$.

ESI-MS: $\quad 269.09[\mathrm{M}+\mathrm{H}]^{+}$

HRMS: $\quad$ found $268.12063[\mathrm{M}]^{+}$

calc. 268.12000

Purification: column chromatography $($ cyclohexane/EtOAc $=2 / 1$ )

Appearance: yellow solid 
Yield: $\quad 16 \mathrm{mg}, 20 \%$. This product contained some photo-inactive impurities, see the spectra in the Supp. Info.

\section{3,8-Bis(methoxymethoxy)-11,12-dihydrodibenzo[c,g]-1,2-diazocine 11}

$2 \mathbf{l}(397 \mathrm{mg}, 1.01 \mathrm{mmol})$ was reacted with $\mathrm{LiAlH}_{4}$ in THF (2.40 M, $\left.8.5 \mathrm{~mL}, 20 \mathrm{mmol}\right)$.

${ }^{1} \mathrm{H}$ NMR (400 MHz, $\left.\mathrm{CDCl}_{3}\right): \delta 6.88(\mathrm{~d}, J=8.4 \mathrm{~Hz}, 2 \mathrm{H}), 6.70(\mathrm{dd}, J=8.4,2.5 \mathrm{~Hz}, 2 \mathrm{H}), 6.54(\mathrm{~d}$, $J=2.5 \mathrm{~Hz}, 2 \mathrm{H}), 5.07$ (s, 4H), $3.42(\mathrm{~s}, 6 \mathrm{H}), 2.96-2.80(\mathrm{~m}, 2 \mathrm{H}), 2.77-2.62(\mathrm{~m}, 2 \mathrm{H})$.

${ }^{13} \mathrm{C}$ NMR (101 MHz, $\left.\mathrm{CDCl}_{3}\right): \delta 156.2,156.0,130.7,121.7,115.1,107.0,94.8,56.2,31.2$.

ESI-MS: $\quad 329.08[\mathrm{M}+\mathrm{H}]^{+}$ $351.02[\mathrm{M}+\mathrm{Na}]^{+}$

HRMS: $\quad$ found $329.14932[\mathrm{M}+\mathrm{H}]^{+}$ calc. $\quad 329.14958$

Purification: gradient sublimation $\left(150{ }^{\circ} \mathrm{C}, 10^{-5} \mathrm{mbar}, 1.5 \mathrm{~h}\right)$

Appearance: yellow solid

Yield: $\quad 40 \mathrm{mg}, 12 \%$

\section{4,7-Difluoro-11,12-dihydrodibenzo[c,g]-1,2-diazocine $1 \mathrm{~m}$}

For the ring closure of $\mathbf{2} \mathbf{m}(0.30 \mathrm{~g}, 1.0 \mathrm{mmol})$, the reagent $\mathrm{LiAlH}(\mathrm{OMe})_{3}$ was first formed from $\mathrm{LiAlH}_{4}$ in THF $(2.40 \mathrm{M}, 8.1 \mathrm{~mL}, 19 \mathrm{mmol})$ and $\mathrm{MeOH}(2.4 \mathrm{~mL}, 58.4 \mathrm{mmol})$ at $0{ }^{\circ} \mathrm{C}$.

${ }^{1} \mathrm{H}$ NMR (400 MHz, $\left.\mathrm{CDCl}_{3}\right): \delta 7.03(\mathrm{td}, J=8.0,5.4 \mathrm{~Hz}, 2 \mathrm{H}), 6.89(\mathrm{t}, J=8.9 \mathrm{~Hz}, 2 \mathrm{H}), 6.77(\mathrm{~d}$, $J=7.6 \mathrm{~Hz}, 2 \mathrm{H}), 3.04-2.89(\mathrm{~m}, 2 \mathrm{H}), 2.88-2.74(\mathrm{~m}, 2 \mathrm{H})$.

${ }^{13} \mathrm{C}$ NMR (101 MHz, $\left.\mathrm{CDCl}_{3}\right): \delta 152.2(\mathrm{~d}, J=249.2 \mathrm{~Hz}), 143.2(\mathrm{~d}, J=13.6 \mathrm{~Hz}), 131.2$ (s), 128.7 $(\mathrm{d}, J=7.9 \mathrm{~Hz}), 124.9$ (d, $J=3.5 \mathrm{~Hz}), 114.5(\mathrm{~d}, J=19.2 \mathrm{~Hz}), 31.5(\mathrm{~s})$.

ESI-MS: $\quad 225.08[\mathrm{M}-\mathrm{F}]^{+}$ $245.07[\mathrm{M}+\mathrm{H}]^{+}$

HRMS: $\quad$ found $245.08897[\mathrm{M}+\mathrm{H}]^{+}$ calc. 245.08848

Purification: column chromatography $(n$-hexane/EtOAc $=4 / 1)$

Appearance: yellow solid

Yield: $\quad 42 \mathrm{mg}, 17 \%$

\section{4,7-Dimethyl-11,12-dihydrodibenzo[c,g]-1,2-diazocine 1n}

2n $(0.10 \mathrm{~g}, 0.33 \mathrm{mmol})$ and $\mathrm{LiAlH}_{4}$ in THF $(2.40 \mathrm{M}, 2.8 \mathrm{~mL}, 6.6 \mathrm{mmol})$ were reacted 
${ }^{1} \mathrm{H}$ NMR (400 MHz, $\left.\mathrm{CDCl}_{3}\right): \delta 6.93-6.91(\mathrm{~m}, 4 \mathrm{H}), 6.80(\mathrm{t}, J=4.4 \mathrm{~Hz}, 2 \mathrm{H}), 2.97-2.90(\mathrm{~m}$, $2 \mathrm{H}), 2.76-2.70(\mathrm{~m}, 2 \mathrm{H}), 2.16(\mathrm{~s}, 6 \mathrm{H})$.

${ }^{13} \mathrm{C}$ NMR (101 MHz, $\left.\mathrm{CDCl}_{3}\right): \delta 154.3,128.9,128.4,127.9,127.3,127.2,32.1,17.1$.

ESI-MS: $\quad 237.16[\mathrm{M}+\mathrm{H}]^{+}$

HRMS: $\quad$ found $237.13862[\mathrm{M}+\mathrm{H}]^{+}$

calc. 237.13863

Purification: gradient sublimation $\left(125^{\circ} \mathrm{C}, 10^{-5} \mathrm{mbar}, 2 \mathrm{~h}\right)$

Appearance: yellow solid, Yield: $5 \mathrm{mg}, 6 \%$ 


\section{UV-Vis spectra and kinetics}

\subsection{UV-Vis spectra of substituted dibenzodiazocines $1 \mathrm{~b}-\mathrm{n}$}

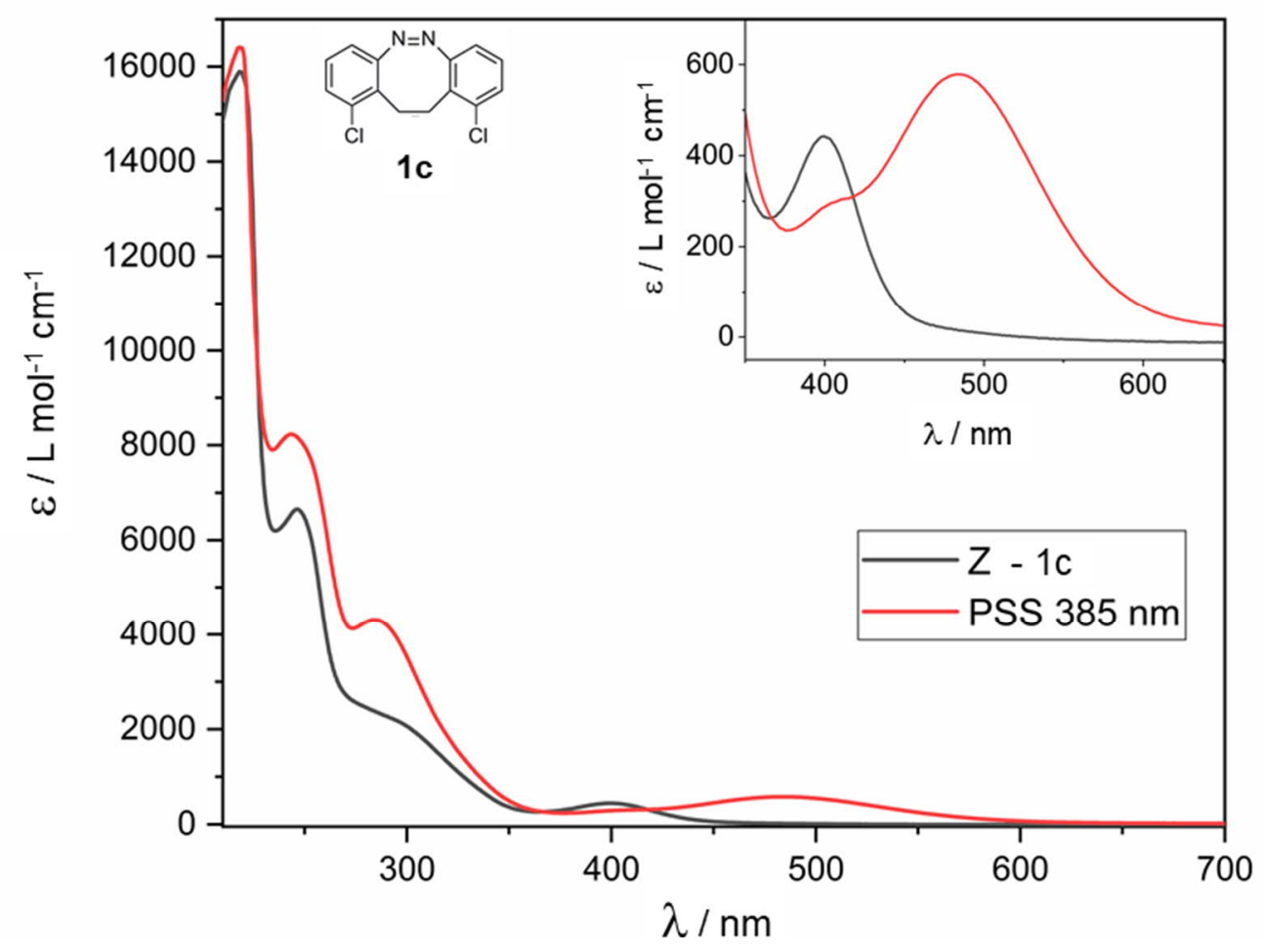

Figure S1. Absorption spectra of 1,10-dichloro-11,12-dihydrodibenzo[c,g]-1,2-diazocine 1c in $\mathrm{MeOH}$ at ground state (Z-isomer; black) and at photo stationary state (PSS; red) at $\lambda=385 \mathrm{~nm}$. Insert shows $n \rightarrow \pi^{*}$ transitions.

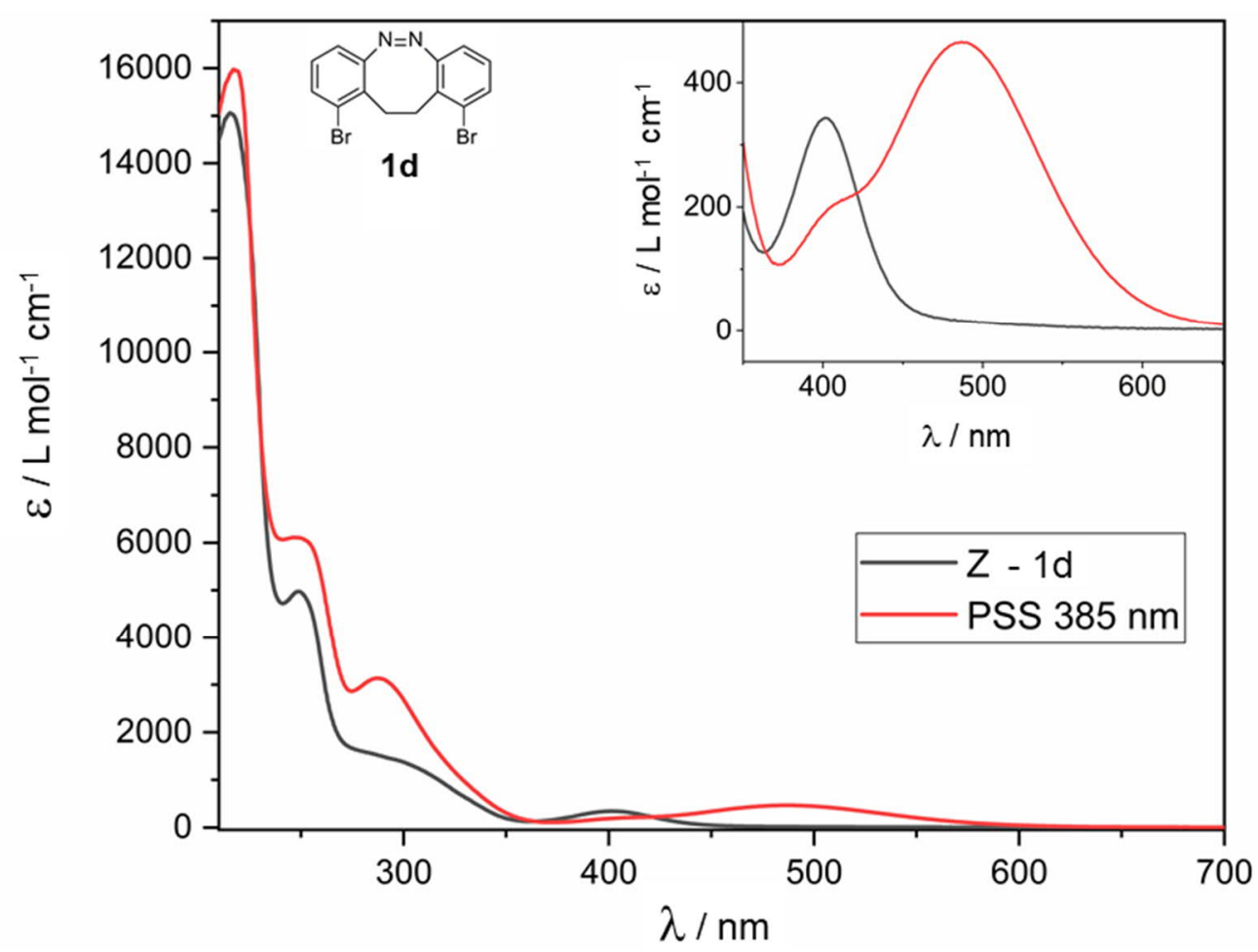

Figure S2. Absorption spectra of 1,10-dibromo-11,12-dihydrodibenzo[c,g]-1,2-diazocine 1d in $\mathrm{MeOH}$ at ground state (Z-isomer; black) and at PSS (red) at $\lambda=385 \mathrm{~nm}$. Insert shows $\mathrm{n} \rightarrow \pi^{*}$ transitions. 


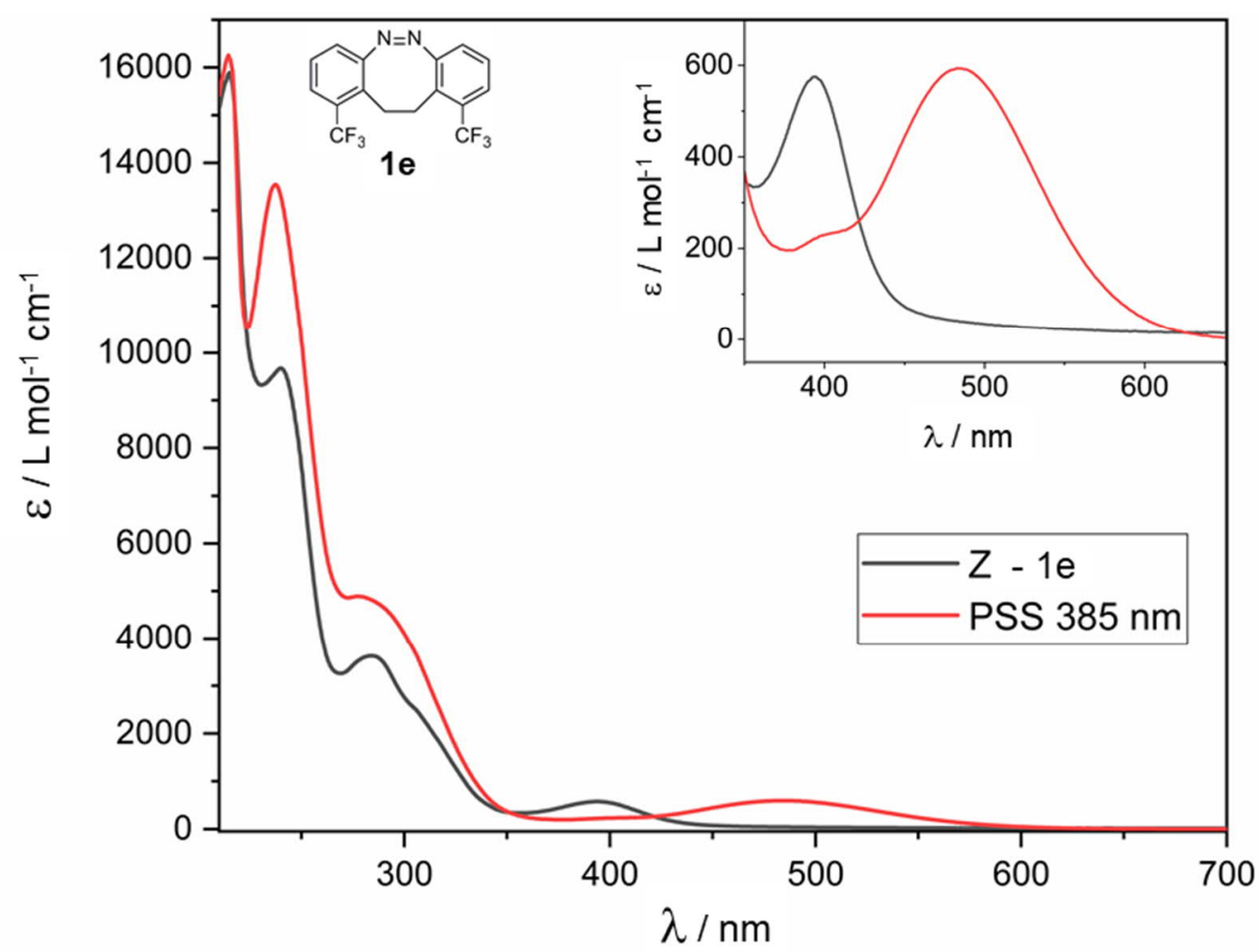

Figure S3. Absorption spectra of 1,10-bis(trifluoromethyl)-11,12-dihydrodibenzo[c,g]-1,2diazocine 1e in $\mathrm{MeOH}$ at ground state (Z-isomer; black) and at PSS (red) at $\lambda=385 \mathrm{~nm}$. Insert shows $n \rightarrow \pi^{*}$ transitions.

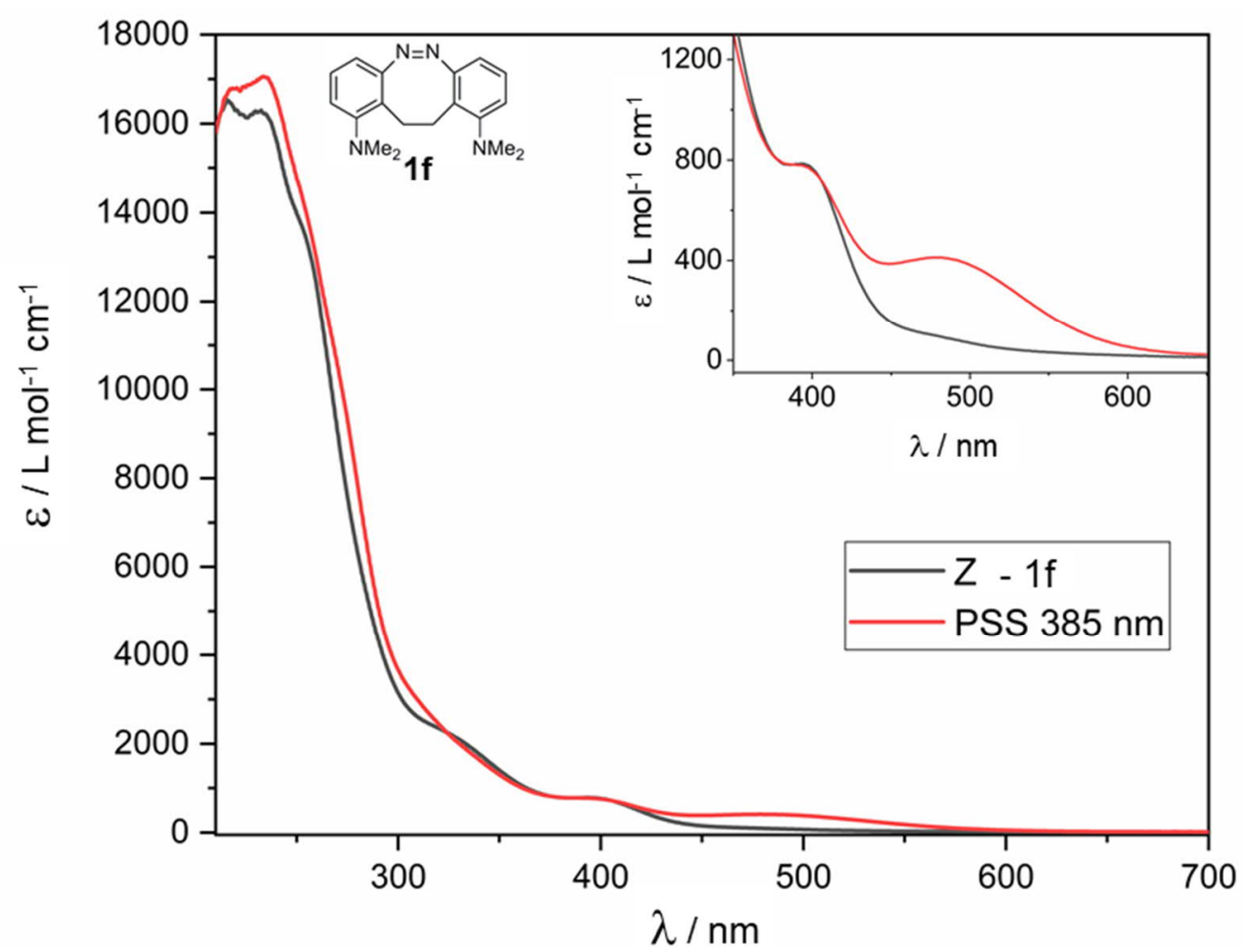

Figure S4. Absorption spectra of 2,9-bis(dimethylamino)-11,12-dihydrodibenzo[c,g]-1,2diazocine $\mathbf{1 f}$ in $\mathrm{MeOH}$ at ground state (Z-isomer; black) and at PSS (red) at $\lambda=385 \mathrm{~nm}$. Insert shows $\mathrm{n} \rightarrow \pi^{*}$ transitions. 


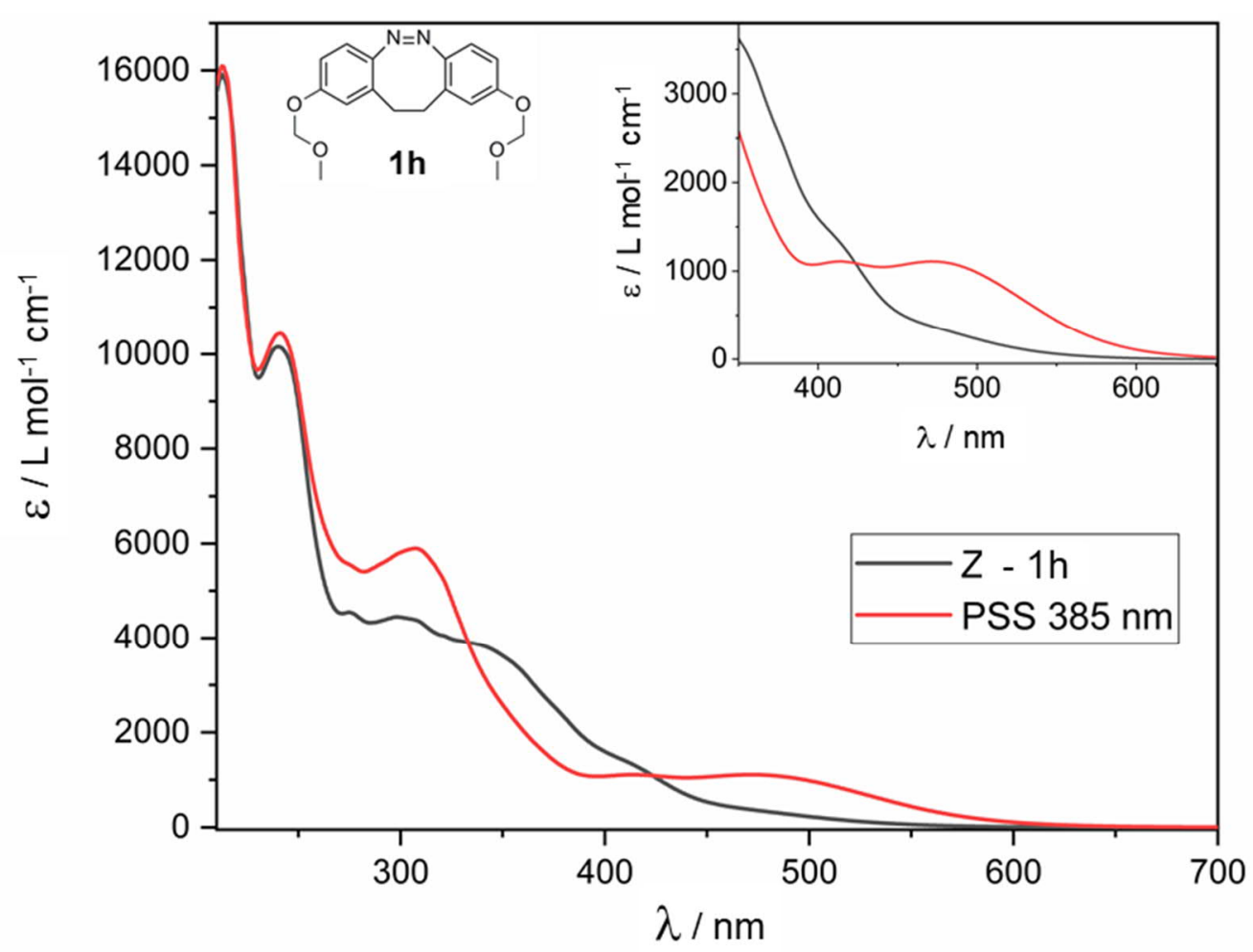

Figure S5. Absorption spectra of 2,9-bis(methoxymethoxy)-11,12-dihydrodibenzo[c,g]-1,2diazocine $1 \mathrm{~h}$ in $\mathrm{MeOH}$ at ground state (Z-isomer; black) and at PSS (red) at $\lambda=385 \mathrm{~nm}$. Insert shows $\mathrm{n} \rightarrow \pi^{*}$ transitions.

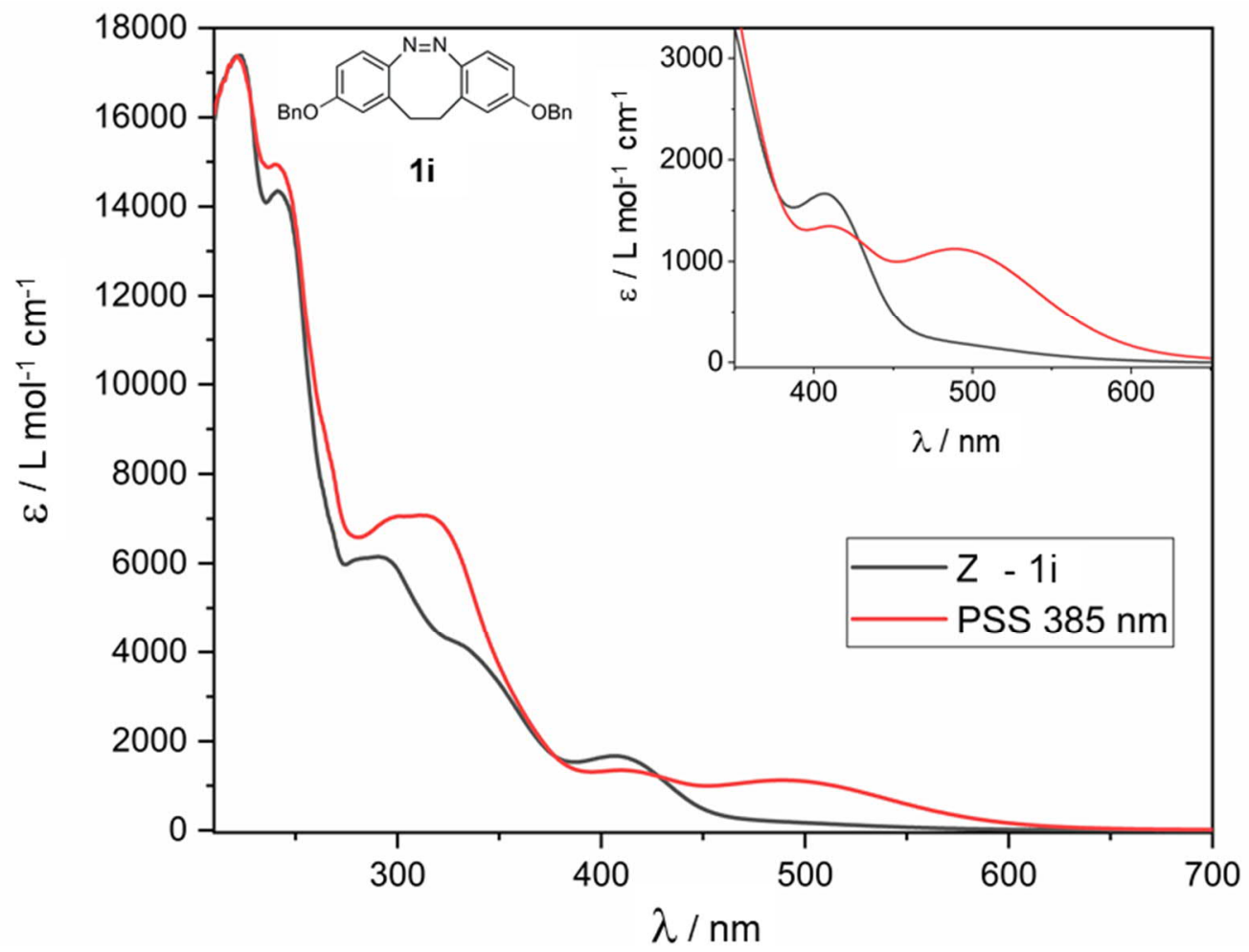

Figure S6. Absorption spectra of 2,9-dibenzyloxy-11,12-dihydrodibenzo[c,g]-1,2-diazocine 1i in $\mathrm{MeOH}$ at ground state (Z-isomer; black) and at PSS (red) at $\lambda=385 \mathrm{~nm}$. Insert shows $\mathrm{n} \rightarrow \pi^{*}$ transitions. 


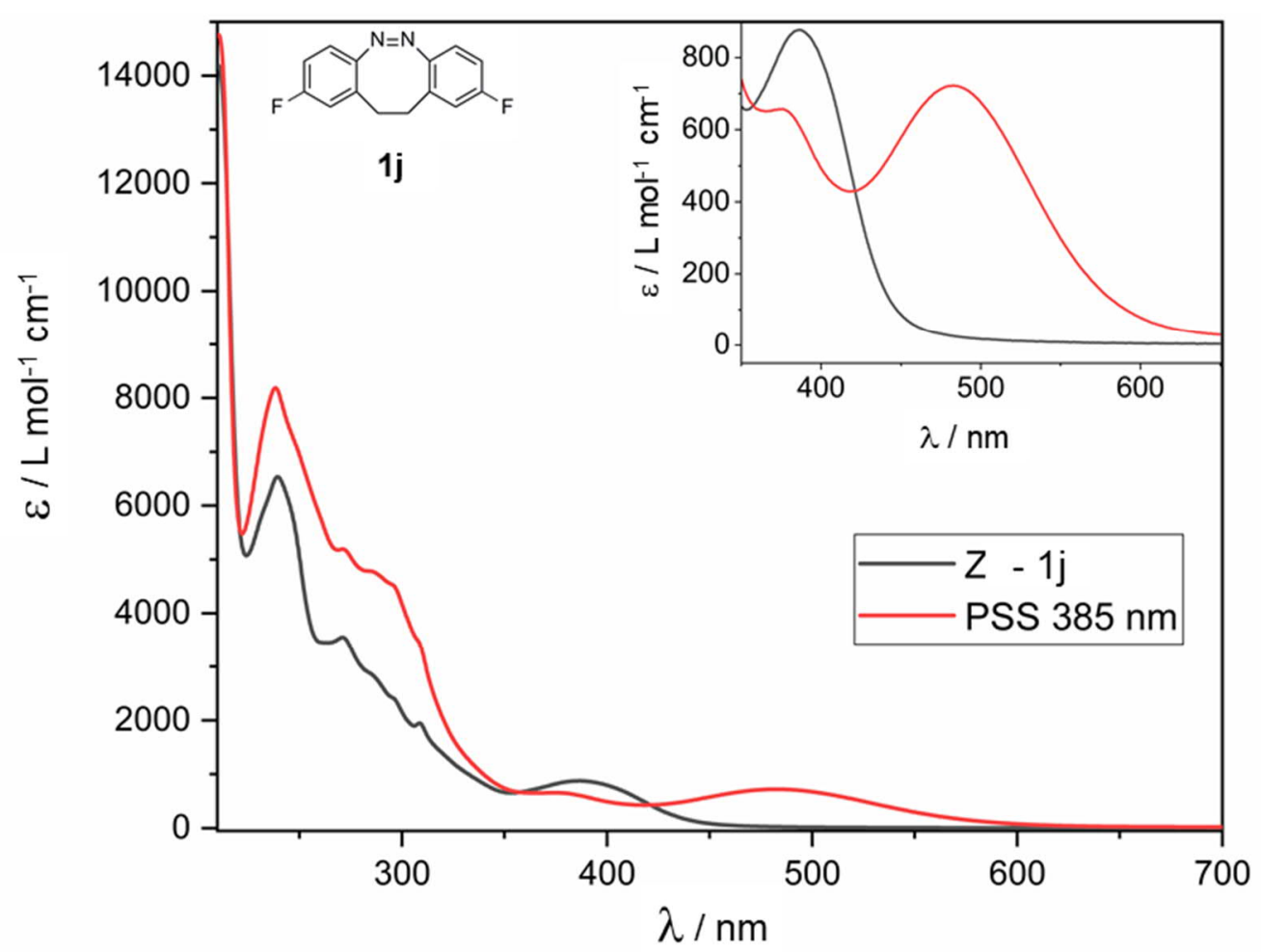

Figure S7. Absorption spectra of 2,9-difluoro-11,12-dihydrodibenzo[c,g]-1,2-diazocine $\mathbf{1 j}$ in $\mathrm{MeOH}$ at ground state (Z-isomer; black) and at PSS (red) at $\lambda=385 \mathrm{~nm}$. Insert shows $\mathrm{n} \rightarrow \pi^{*}$ transitions.

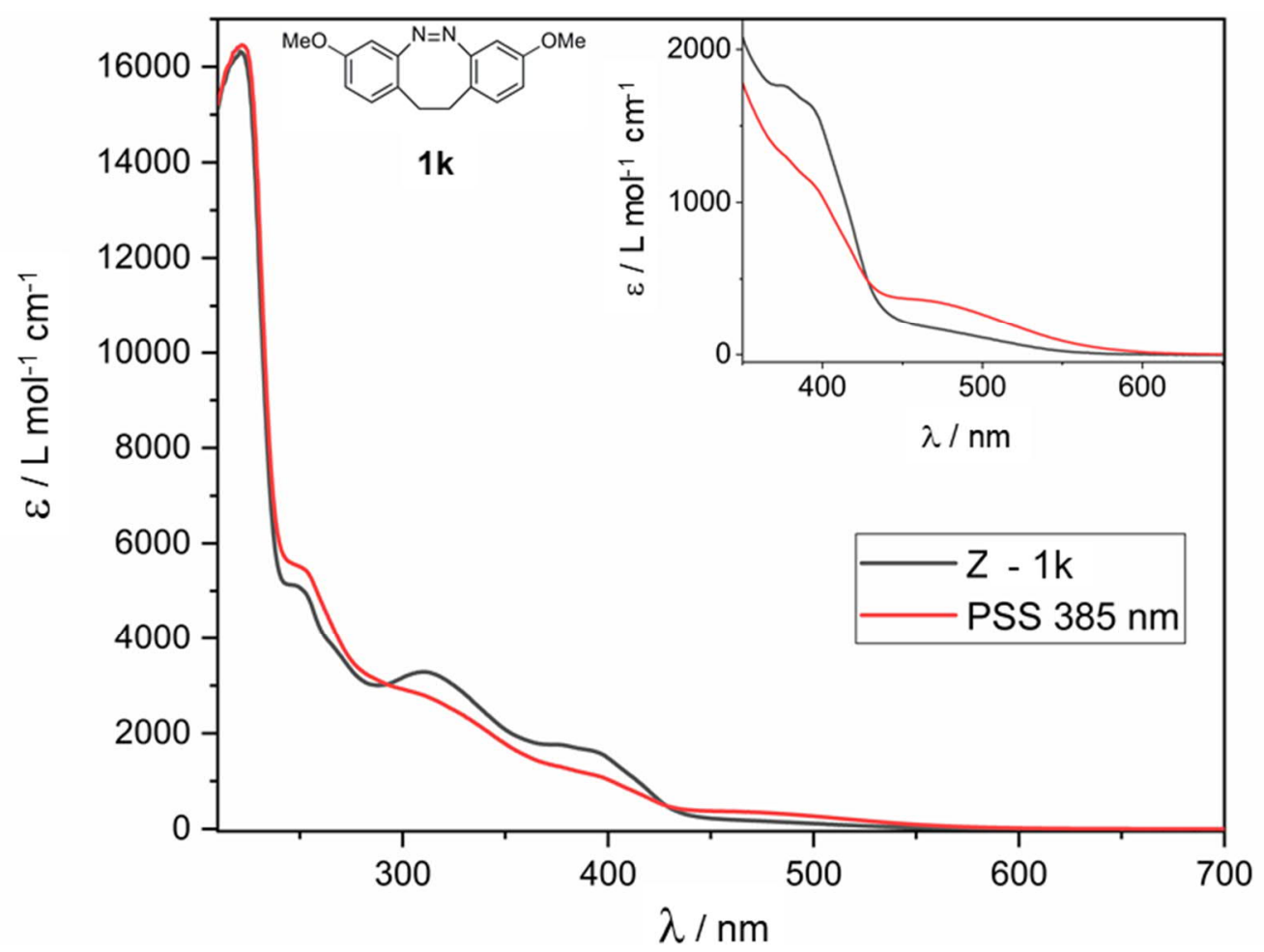

Figure S8. Absorption spectra of 3,8-dimethoxy-11,12-dihydrodibenzo[c,g]-1,2-diazocine $\mathbf{1 k}$ in $\mathrm{MeOH}$ at ground state (Z-isomer; black) and at PSS (red) at $\lambda=385 \mathrm{~nm}$. Insert show $\mathrm{n} \rightarrow \pi^{*}$ transitions. 


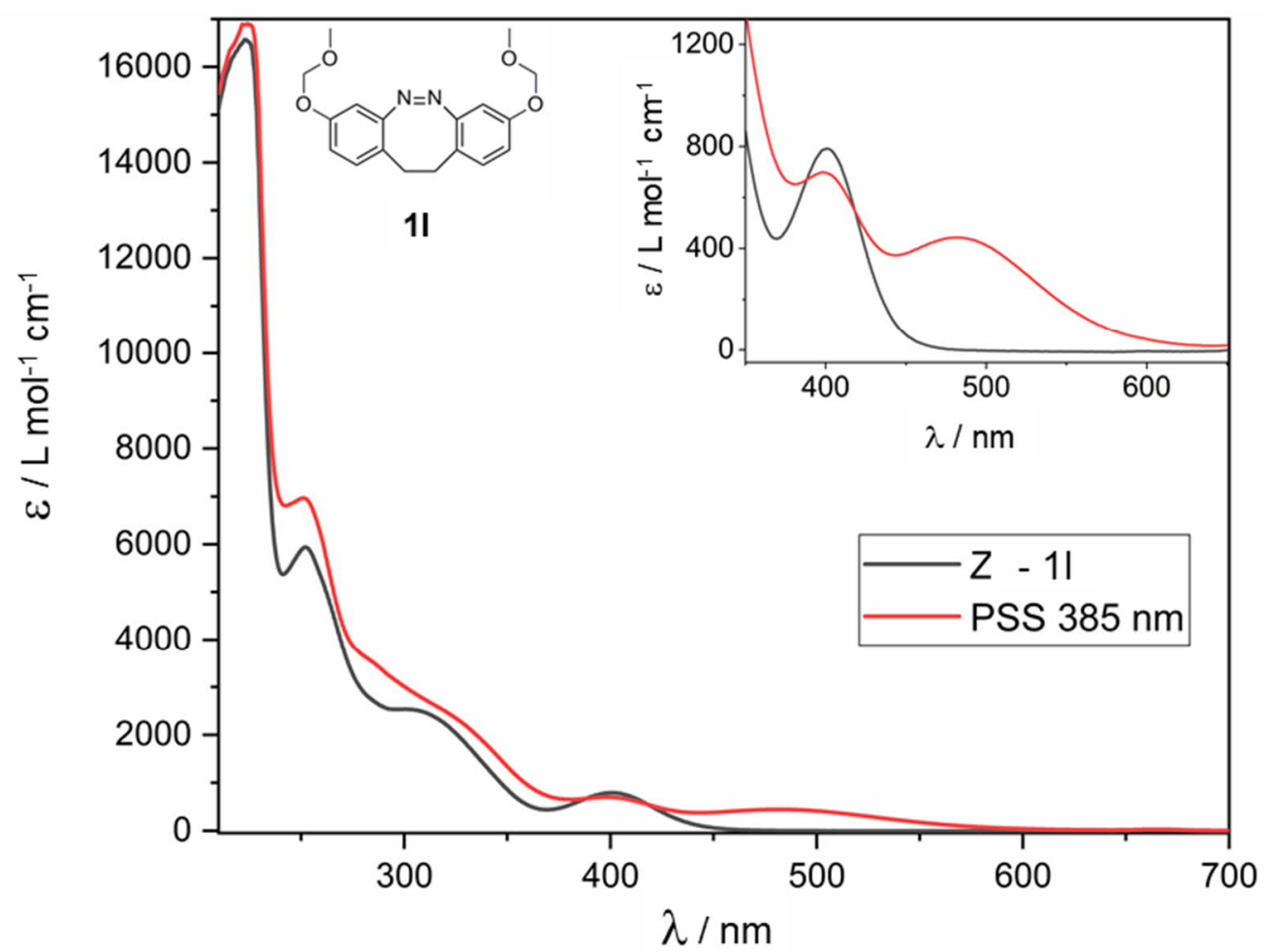

Figure S9. Absorption spectra of 3,8-bis(methoxymethoxy)-11,12-dihydrodibenzo[c,g]-1,2diazocine 11 in $\mathrm{MeOH}$ at ground state (Z-isomer; black) and at PSS (red) at $\lambda=385 \mathrm{~nm}$. Insert show $\mathrm{n} \rightarrow \pi^{*}$ transitions.

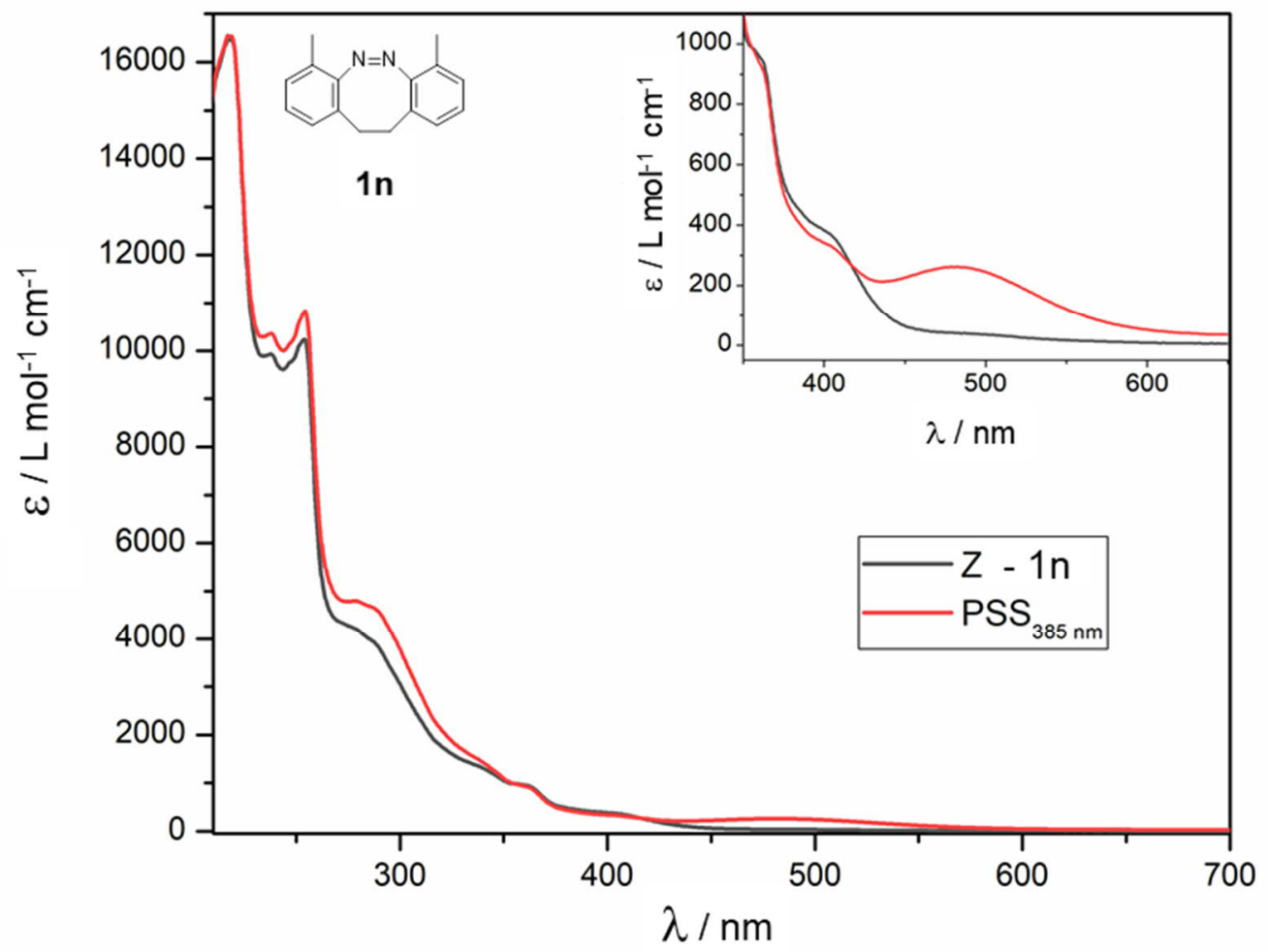

Figure S10. Absorption spectra of 4,10-dimethyl-11,12-dihydrodibenzo[c,g]-1,2-diazocine 1 n in $\mathrm{MeOH}$ at ground state (Z-isomer; black) and at PSS (red) at $\lambda=385 \mathrm{~nm}$. Insert show $\mathrm{n} \rightarrow \pi^{*}$ transitions. 


\subsection{Determination of thermal half-life of the $E$-isomers}

The thermal reisomerization reaction from the $E$ - to the $Z$-isomer was assumed as a first order reaction:

$$
\ln \left(\frac{[E]_{0}}{[E]_{t}}\right)=k t
$$

with the reaction constant $\mathrm{k}$ and the concentration of the $E$-isomer [E].

In consideration of Beer's law, equation (1) can be converted to:

$$
\ln \left(\frac{A_{\infty}-A_{0}}{A_{\infty}-A_{t}}\right)=k t
$$

with the absorbance A of the $E$-isomer at $\lambda=480 \mathrm{~nm}$.

The thermal half-life $\tau_{1 / 2}$ is given by:

$$
\tau_{1 / 2}=\frac{\ln (2)}{k}
$$

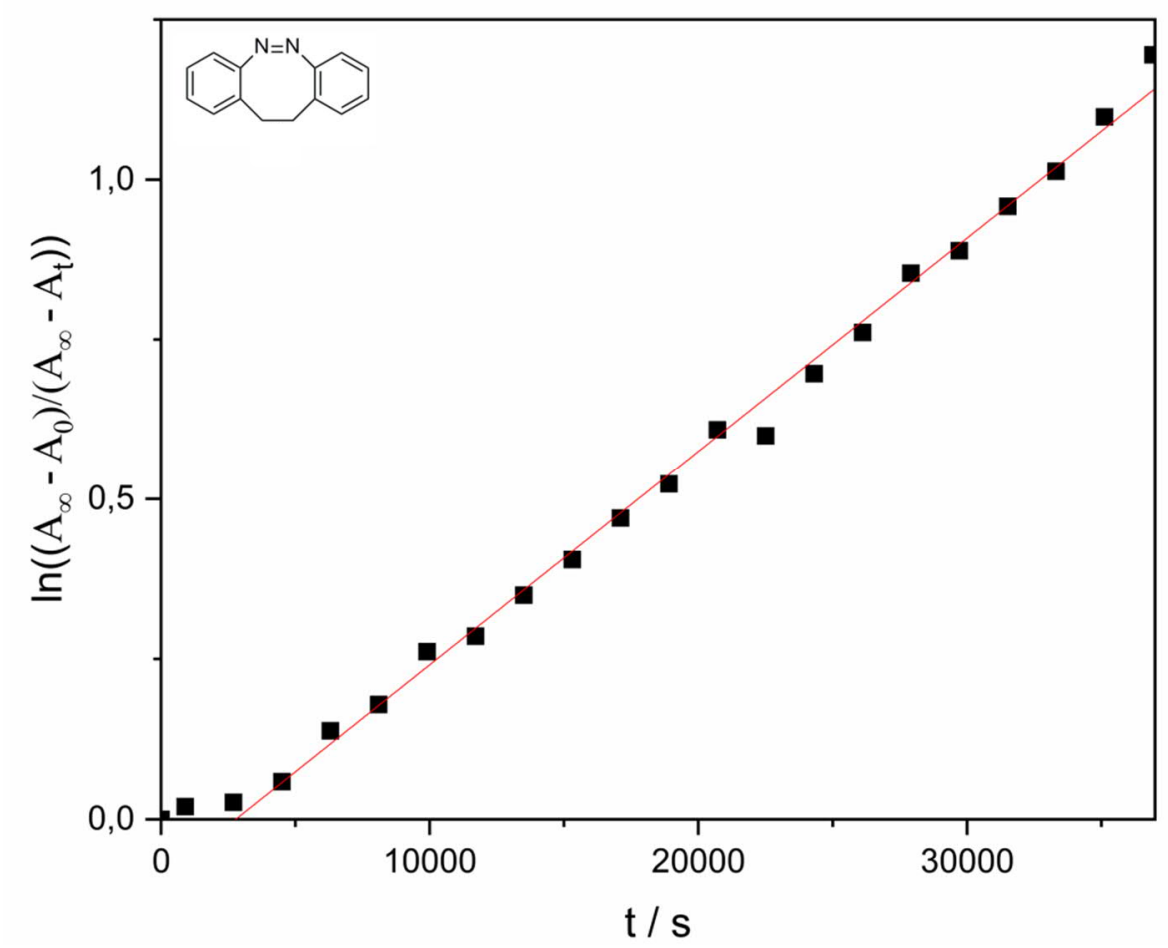

Figure S11. Logarithmized absorbance plotted as a function of time in seconds of 11,12dihydrodibenzo[ $c, g]$-1,2-diazocine 1a in methanol. The slope of the linear fit (in red) gives the reaction constant $\mathrm{k}=(3.63 \pm 0.05) \times 10^{-5} \mathrm{~s}^{-1}\left(\tau_{1 / 2}=5.30 \pm 0.07 \mathrm{~h}\right)$. 


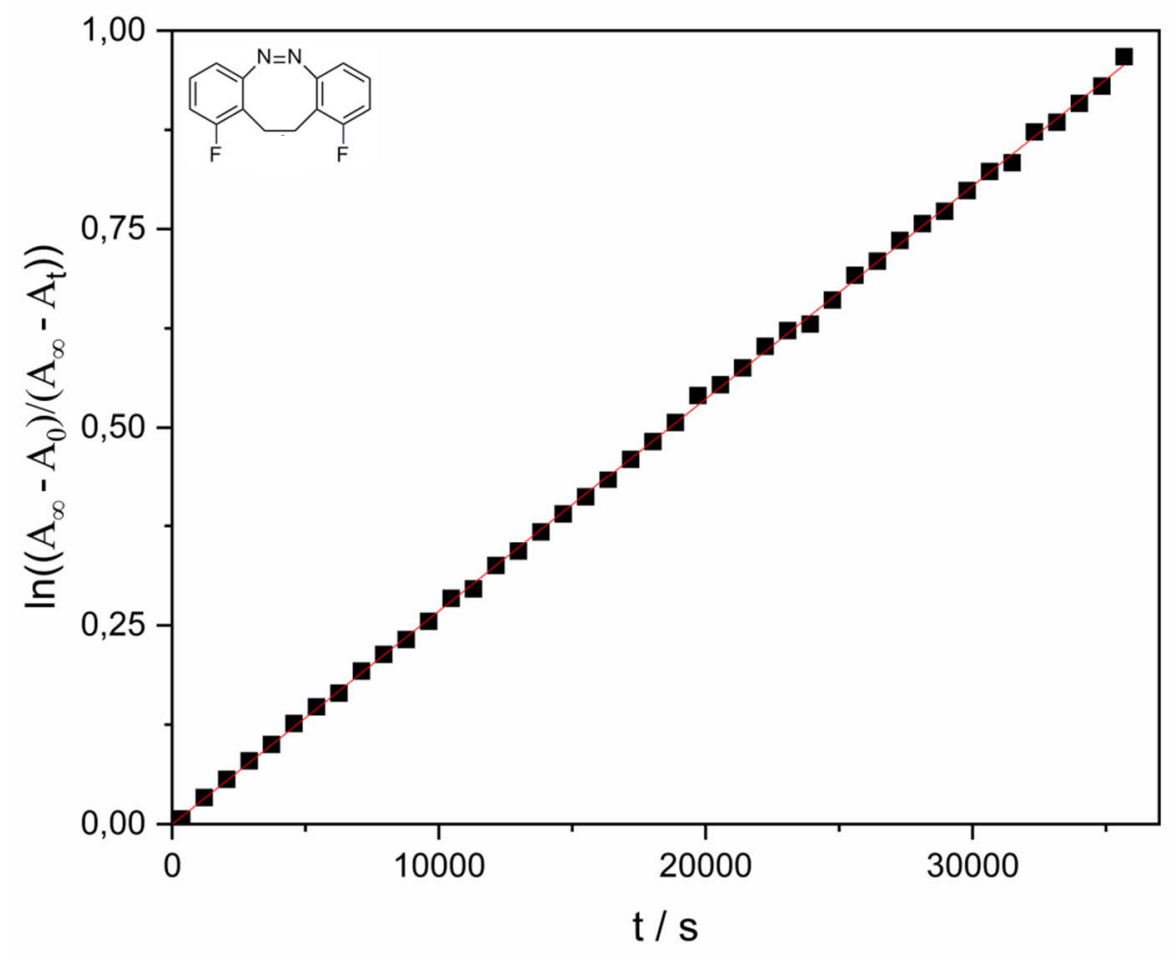

Figure S12. Logarithmized absorbance plotted as a function of time in seconds of 1,10difluoro-11,12-dihydrodibenzo[c,g]-1,2-diazocine $\mathbf{1 b}$ in methanol. The slope of the linear fit (in red) gives the reaction constant $\mathrm{k}=(2.682 \pm 0.007) \times 10^{-5} \mathrm{~s}^{-1}\left(\tau_{1 / 2}=7.18 \pm 0.02 \mathrm{~h}\right)$.

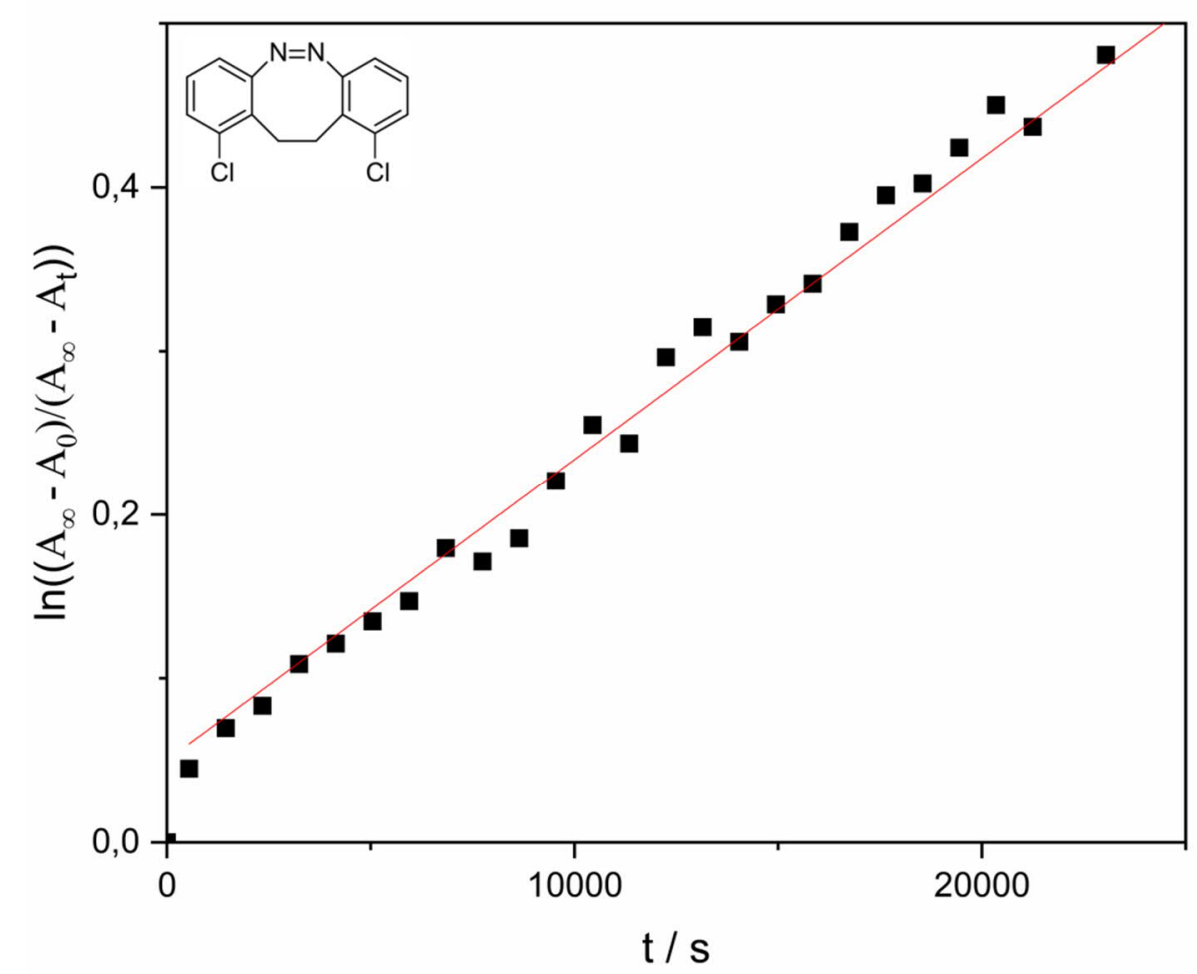

Figure S13. Logarithmized absorbance plotted as a function of time in seconds of 1,10dichloro-11,12-dihydrodibenzo[c,g]-1,2-diazocine 1c in methanol. The slope of the linear fit (in red) gives the reaction constant $\mathrm{k}=(1.84 \pm 0.03) \times 10^{-5} \mathrm{~s}^{-1}\left(\tau_{1 / 2}=10.5 \pm 0.2 \mathrm{~h}\right)$. 


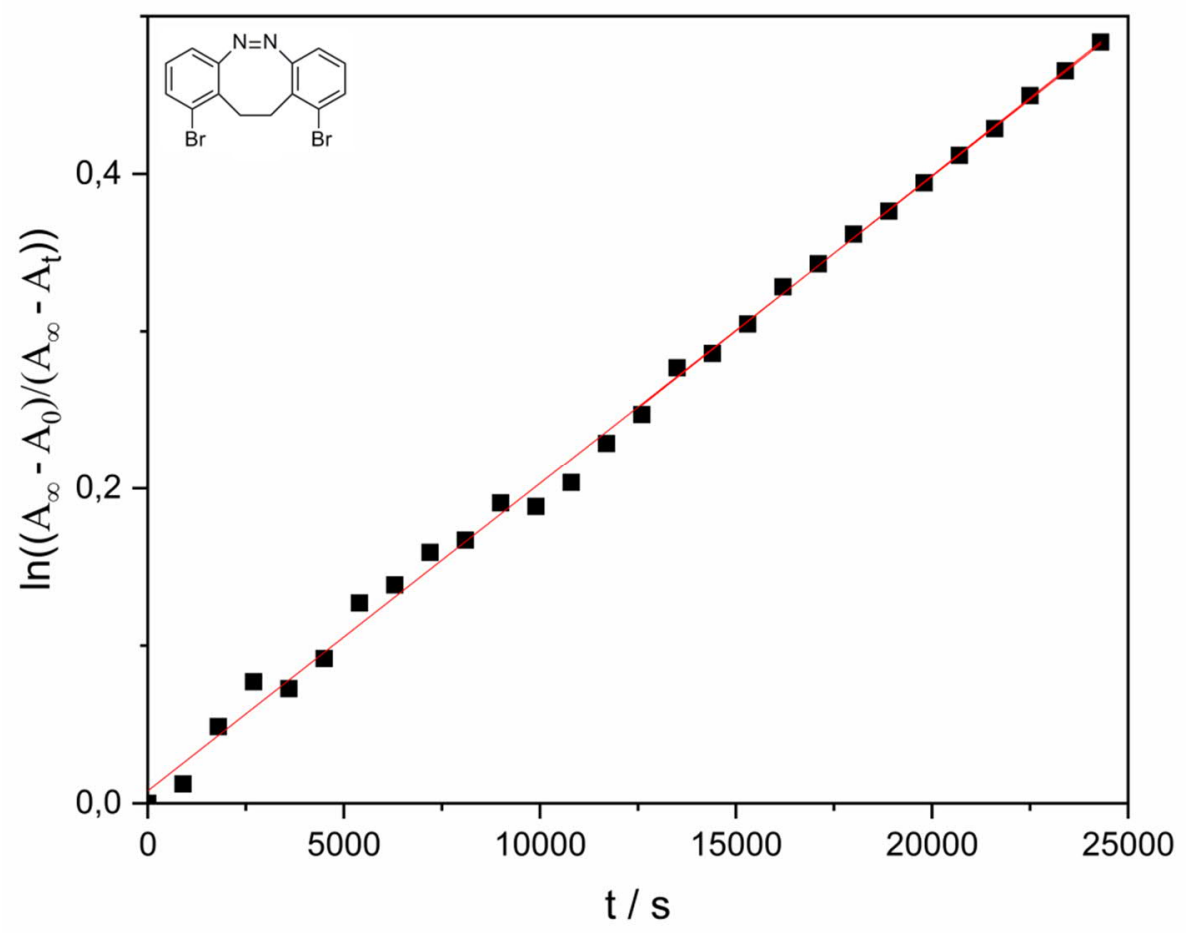

Figure S 14. Logarithmized absorbance plotted as a function of time in seconds of 1,10dibromo-11,12-dihydrodibenzo[c,g]-1,2-diazocine 1d in methanol. The slope of the linear fit (in red) gives the reaction constant $\mathrm{k}=(1.95 \pm 0.02) \times 10^{-5} \mathrm{~s}^{-1}\left(\tau_{1 / 2}=9.87 \pm 0.10 \mathrm{~h}\right)$.

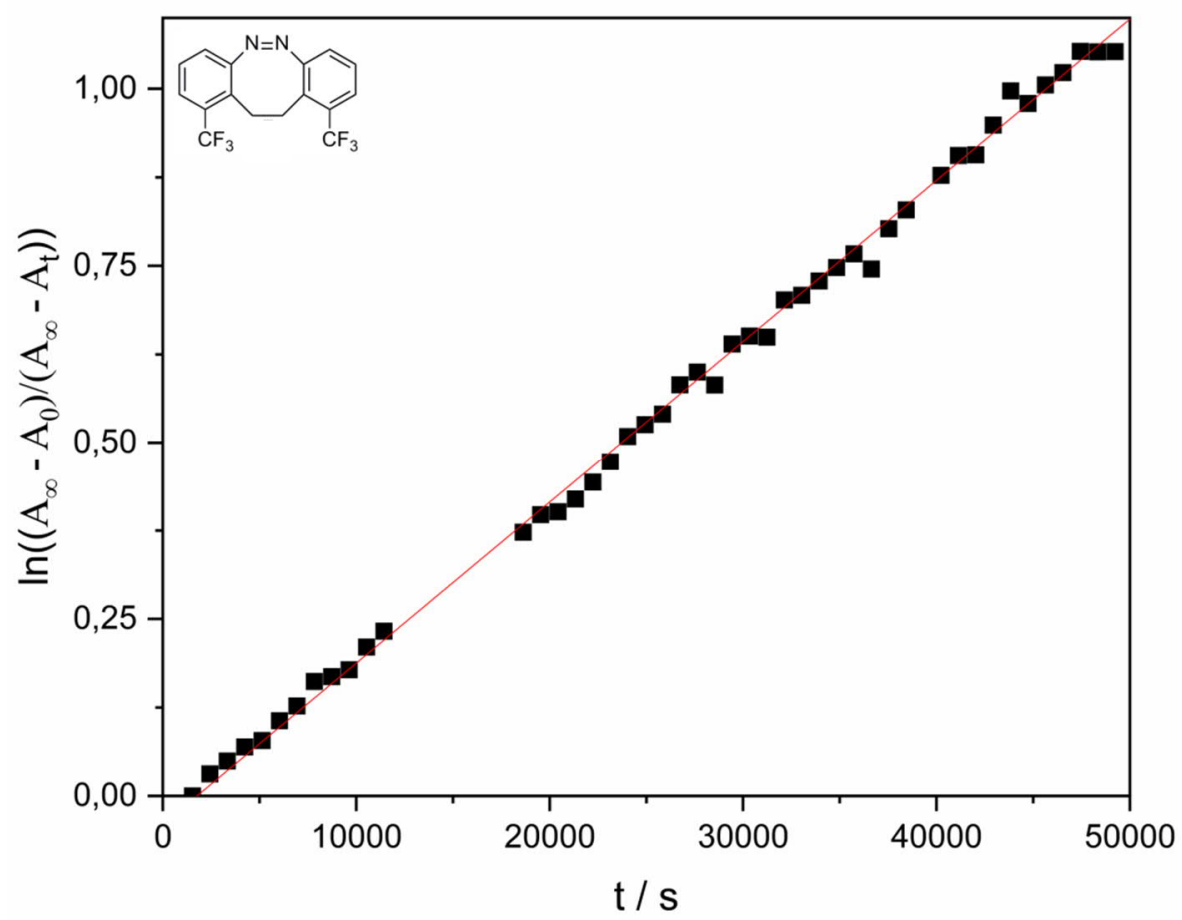

Figure S15. Logarithmized absorbance plotted as a function of time in seconds of 1,10bis(trifluoromethyl)-11,12-dihydrodibenzo[c,g]-1,2-diazocine 1e in methanol. The slope of the linear fit (in red) gives the reaction constant $\mathrm{k}=(2.277 \pm 0.013) \times 10^{-5} \mathrm{~s}^{-1}\left(\tau_{1 / 2}=8.46 \pm 0.05 \mathrm{~h}\right)$. 


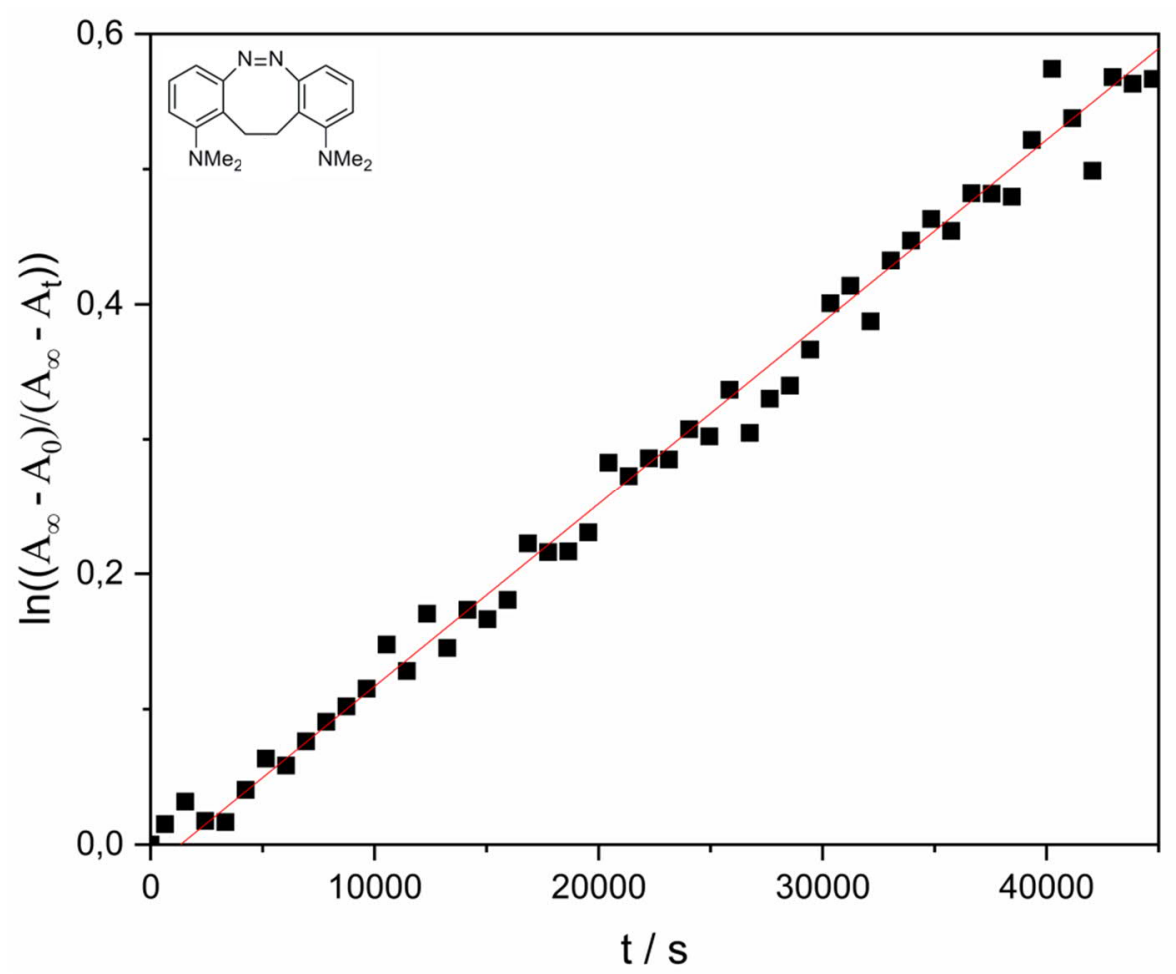

Figure S16. Logarithmized absorbance plotted as a function of time in seconds of 1,10di(dimethylamino)-11,12-dihydrodibenzo[c,g]-1,2-diazocine $\mathbf{1 f}$ in methanol. The slope of the linear fit (in red) gives the reaction constant $\mathrm{k}=(1.349 \pm 0.015) \times 10^{-5} \mathrm{~s}^{-1}\left(\tau_{1 / 2}=14.3 \pm 0.2 \mathrm{~h}\right)$.

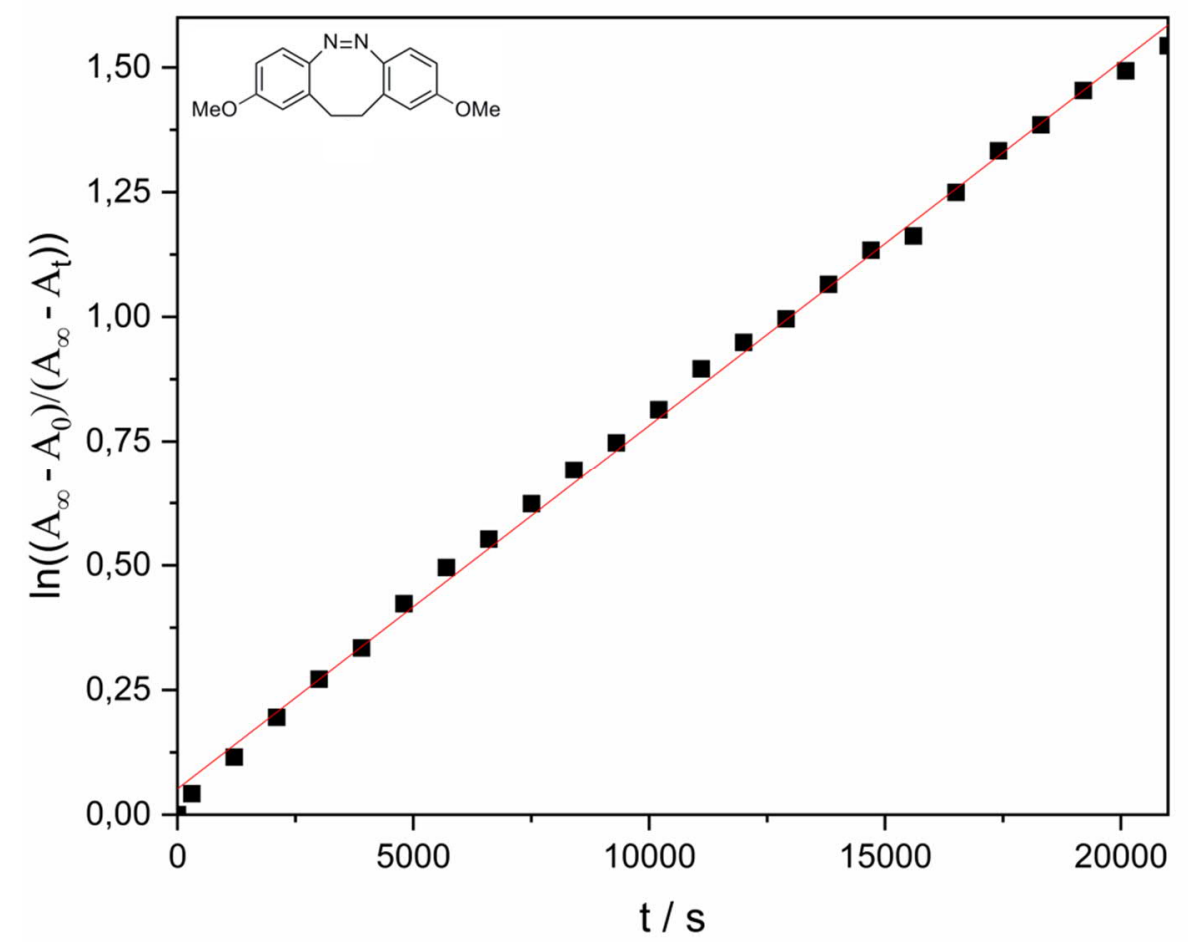

Figure S17. Logarithmized absorbance plotted as a function of time in seconds of 2,9dimethoxy-11,12-dihydrodibenzo[c,g]-1,2-diazocine $1 \mathrm{~g}$ in methanol. The slope of the linear fit (in red) gives the reaction constant $\mathrm{k}=(7.30 \pm 0.08) \times 10^{-5} \mathrm{~s}^{-1}\left(\tau_{1 / 2}=2.64 \pm 0.03 \mathrm{~h}\right)$. 


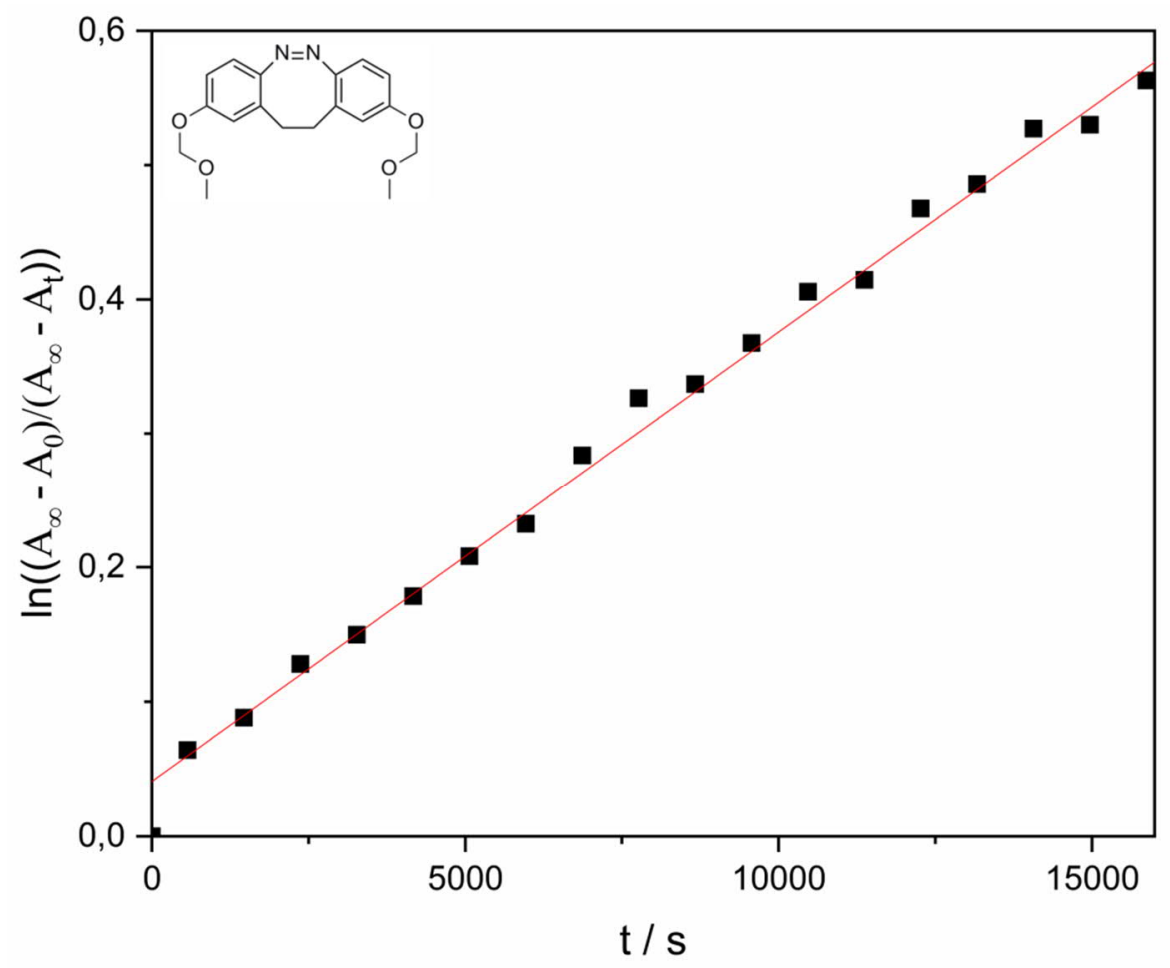

Figure S18. Logarithmized absorbance plotted as a function of time in seconds of 2,9bis(methoxymethoxy)-11,12-dihydrodibenzo[c,g]-1,2-diazocine $\mathbf{1 h}$ in methanol. The slope of the linear fit (in red) gives the reaction constant $\mathrm{k}=(8.70 \pm 0.06) \times 10^{-5} \mathrm{~s}^{-1}\left(\tau_{1 / 2}=2.21 \pm 0.02 \mathrm{~h}\right)$.

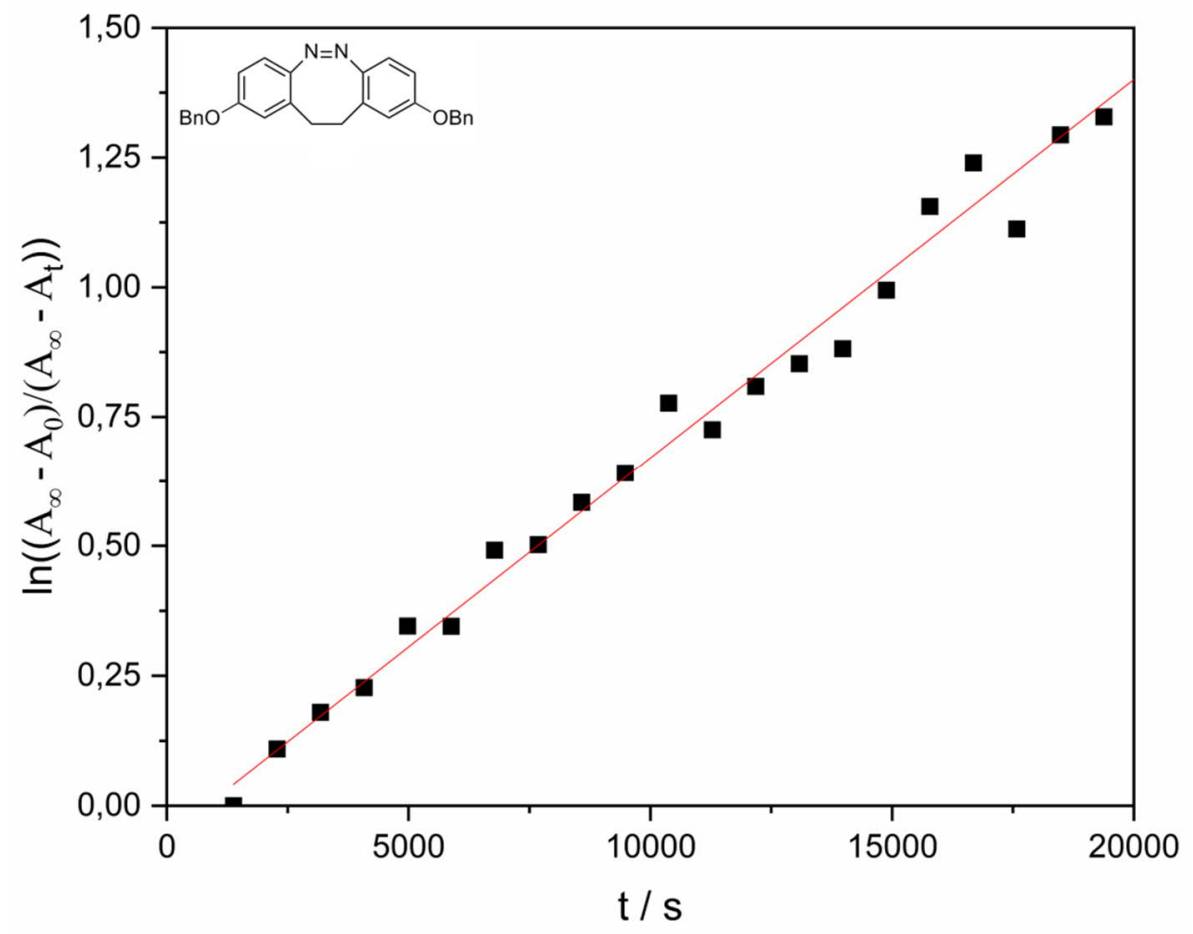

Figure S19. Logarithmized absorbance plotted as a function of time in seconds of 2,9dibenzyloxy-11,12-dihydrodibenzo[c,g]-1,2-diazocine $\mathbf{1 j}$ in methanol. The slope of the linear fit (in red) gives the reaction constant $\mathrm{k}=(7.30 \pm 0.17) \times 10^{-5} \mathrm{~s}^{-1}\left(\tau_{1 / 2}=2.64 \pm 0.06 \mathrm{~h}\right)$. 


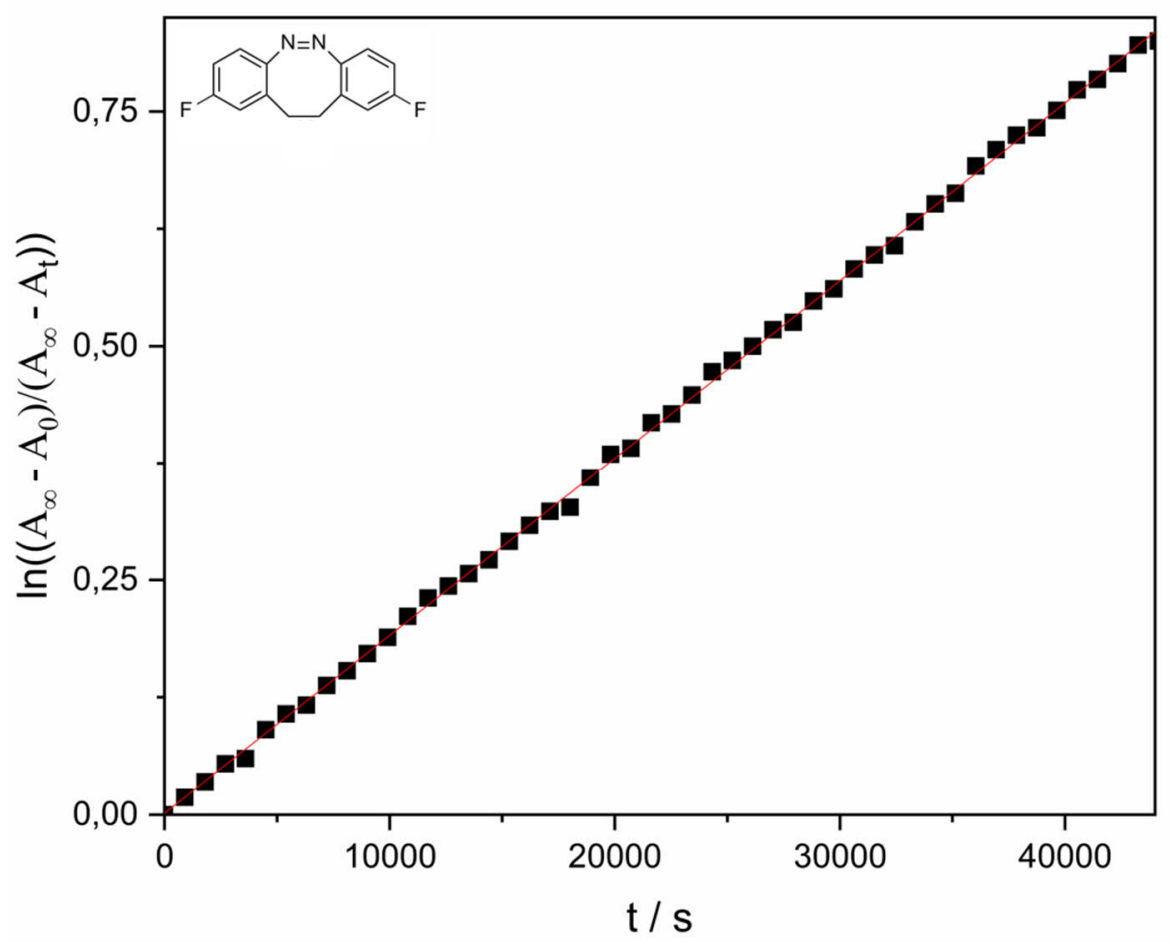

Figure S20. Logarithmized absorbance plotted as a function of time in seconds of 2,9-difluoro11,12-dihydrodibenzo[c,g]-1,2-diazocine $\mathbf{1 k}$ in methanol. The slope of the linear fit (in red) gives the reaction constant $\mathrm{k}=(1.894 \pm 0.006) \times 10^{-5} \mathrm{~s}^{-1}\left(\tau_{1 / 2}=10.2 \pm 0.1 \mathrm{~h}\right)$.

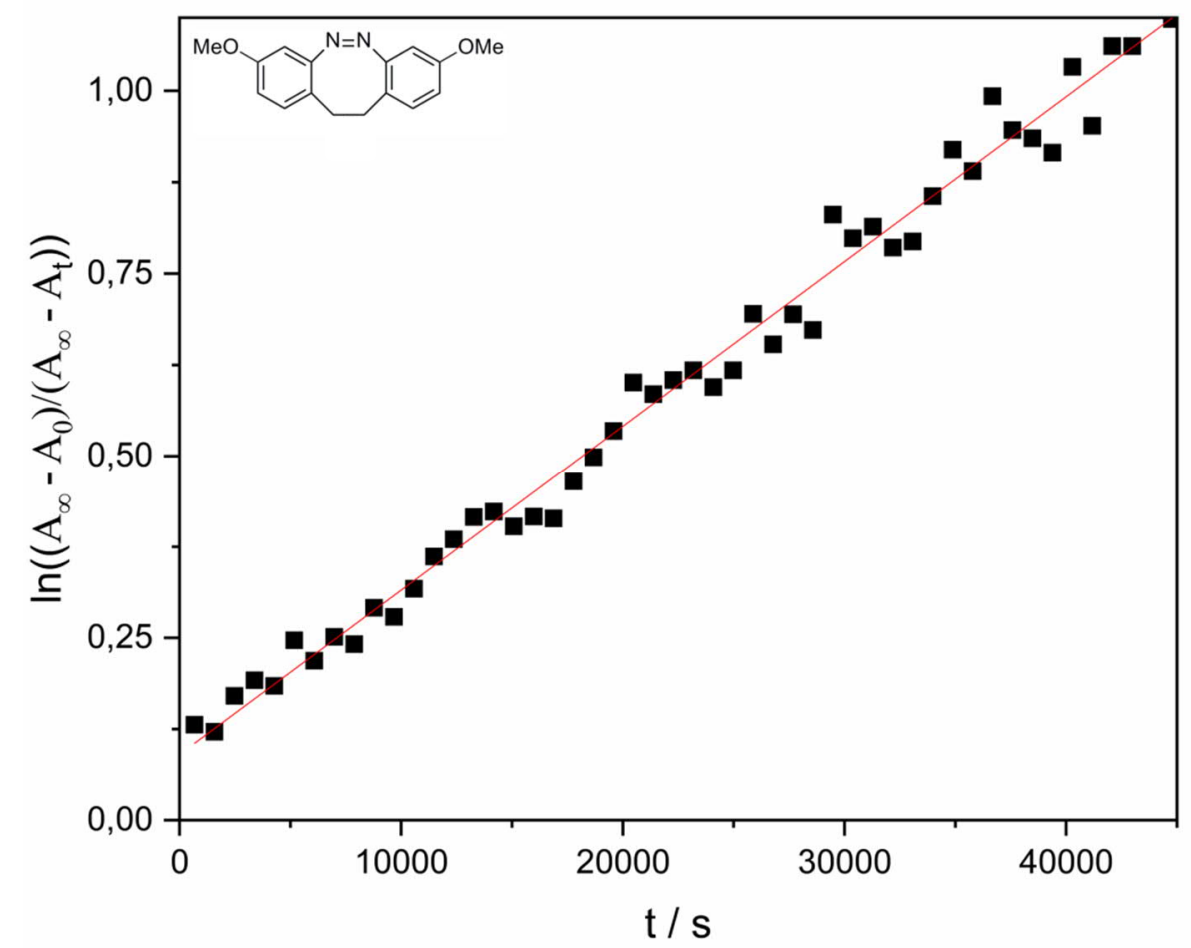

Figure S21. Logarithmized absorbance plotted as a function of time in seconds of 3,8dimethoxy-11,12-dihydrodibenzo[c,g]-1,2-diazocine 1k in methanol. The slope of the linear fit (in red) gives the reaction constant $\mathrm{k}=(2.25 \pm 0.04) \times 10^{-5} \mathrm{~s}^{-1}\left(\tau_{1 / 2}=8.56 \pm 0.15 \mathrm{~h}\right)$. 


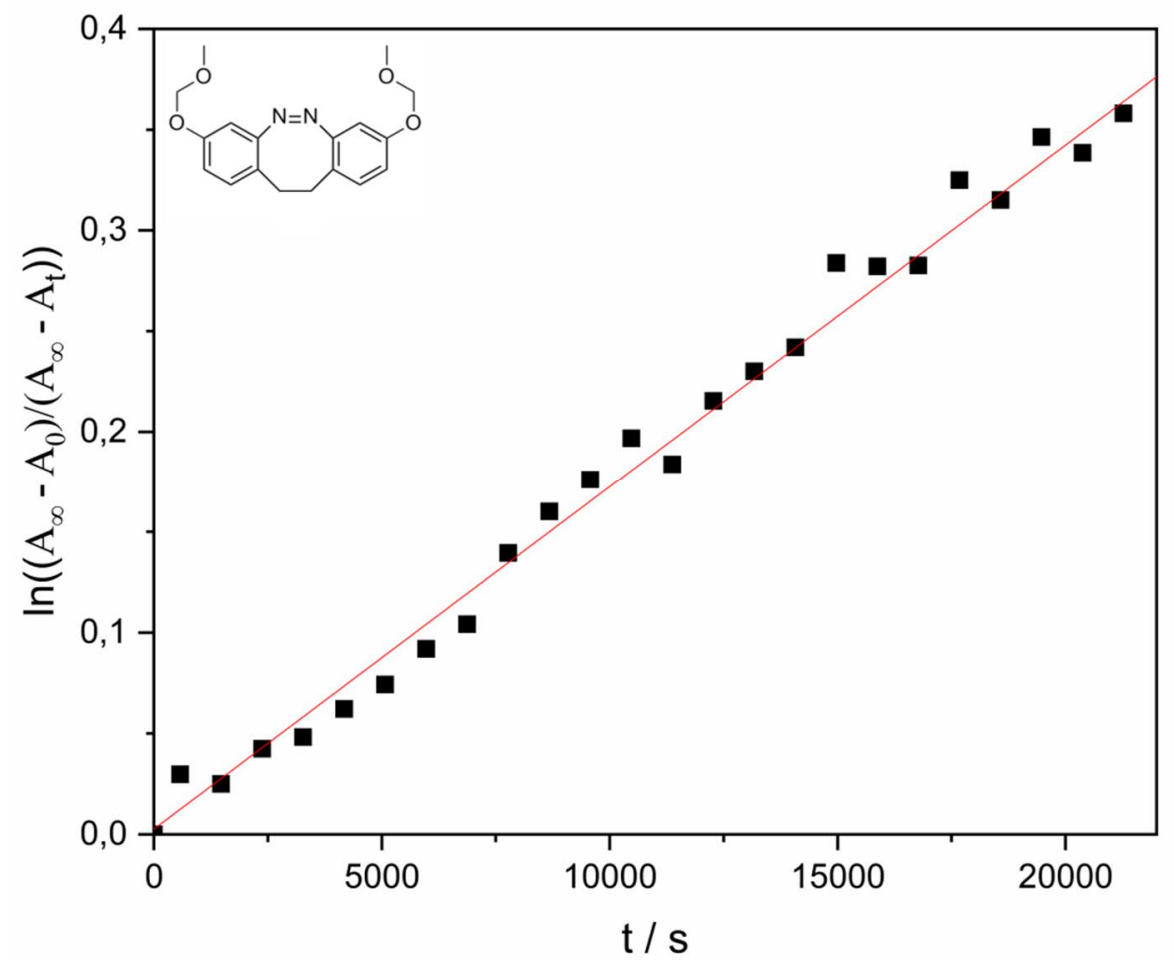

Figure S22. Logarithmized absorbance plotted as a function of time in seconds of 3,8bis(methoxymethoxy)-11,12-dihydrodibenzo[c,g]-1,2-diazocine 11 in methanol. The slope of the linear fit (in red) gives the reaction constant $\mathrm{k}=(1.70 \pm 0.02) \times 10^{-5} \mathrm{~s}^{-1}\left(\tau_{1 / 2}=11.3 \pm 0.2 \mathrm{~h}\right)$.

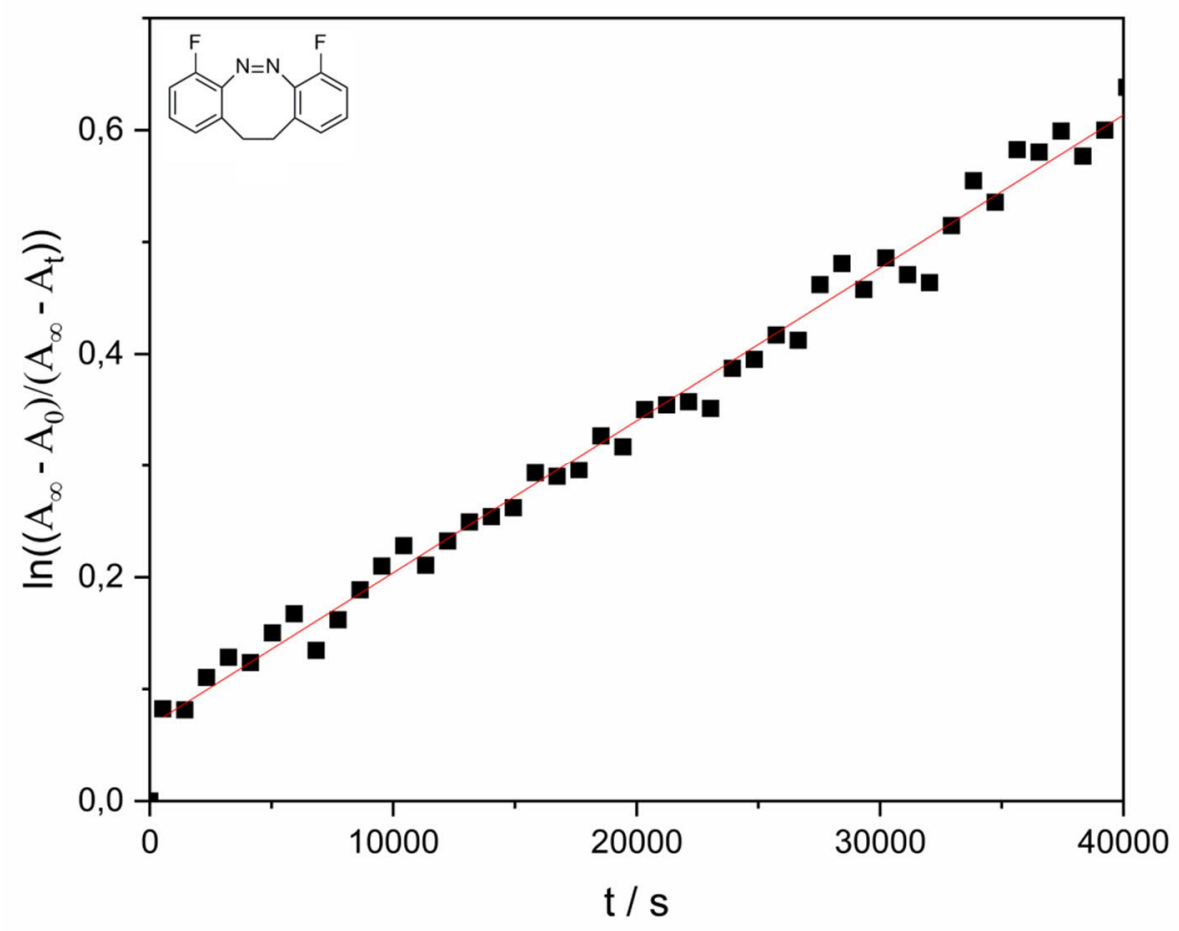

Figure S23. Logarithmized absorbance plotted as a function of time in seconds of 4,7-difluoro11,12-dihydrodibenzo[ $c, g]$-1,2-diazocine $\mathbf{1 m}$ in methanol. The slope of the linear fit (in red) gives the reaction constant $\mathrm{k}=(1.37 \pm 0.02) \times 10^{-5} \mathrm{~s}^{-1}\left(\tau_{1 / 2}=14.1 \pm 0.2 \mathrm{~h}\right)$. 


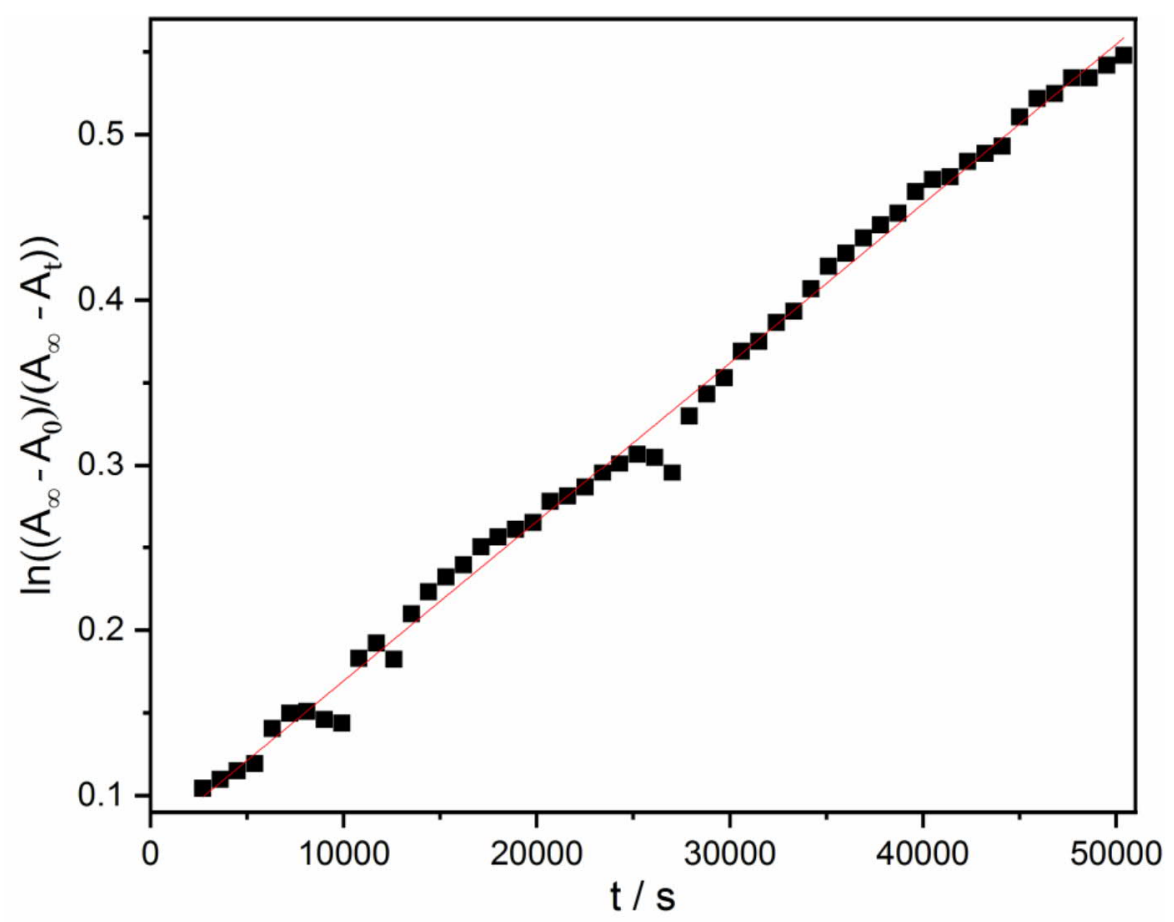

Figure S24. Logarithmized absorbance plotted as a function of time in seconds of 4,7-dimethyl11,12-dihydrodibenzo[c,g]-1,2-diazocine $\mathbf{1 n}$ in methanol. The slope of the linear fit (in red) gives the reaction constant $\mathrm{k}=(9.63 \pm 0.09) \times 10^{-6} \mathrm{~s}^{-1}\left(\tau_{1 / 2}=20.0 \pm 0.2 \mathrm{~h}\right)$. 


\section{NMR spectra of synthesized compounds}
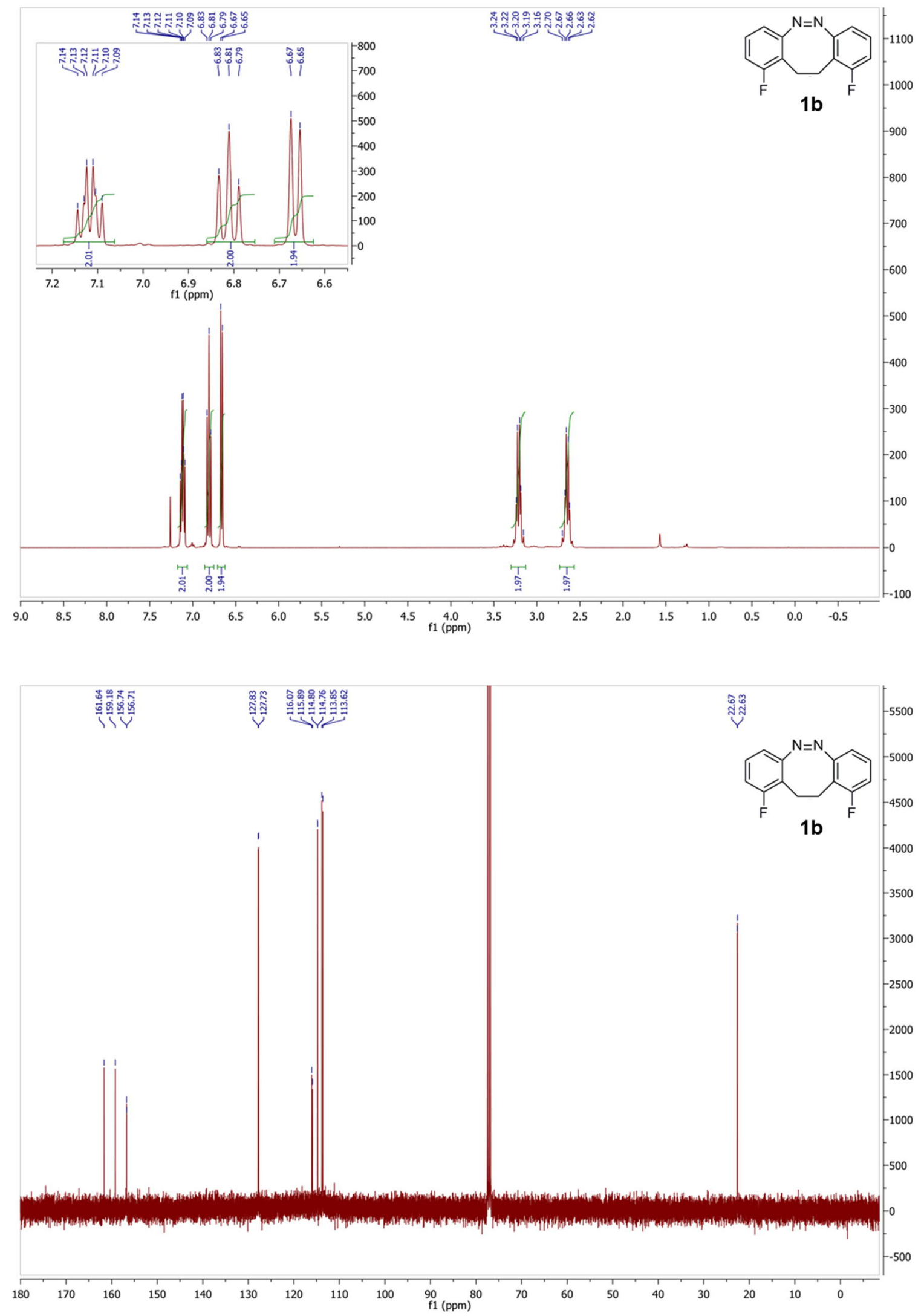

Figure S25. ${ }^{1} \mathrm{H}$ NMR (400 MHz) and ${ }^{13} \mathrm{C}$ NMR (101 MHz) spectra of 1,10-difluoro-11,12dihydrodibenzo[c,g]-1,2-diazocine $\mathbf{1 b}$ in $\mathrm{CDCl}_{3}$. 

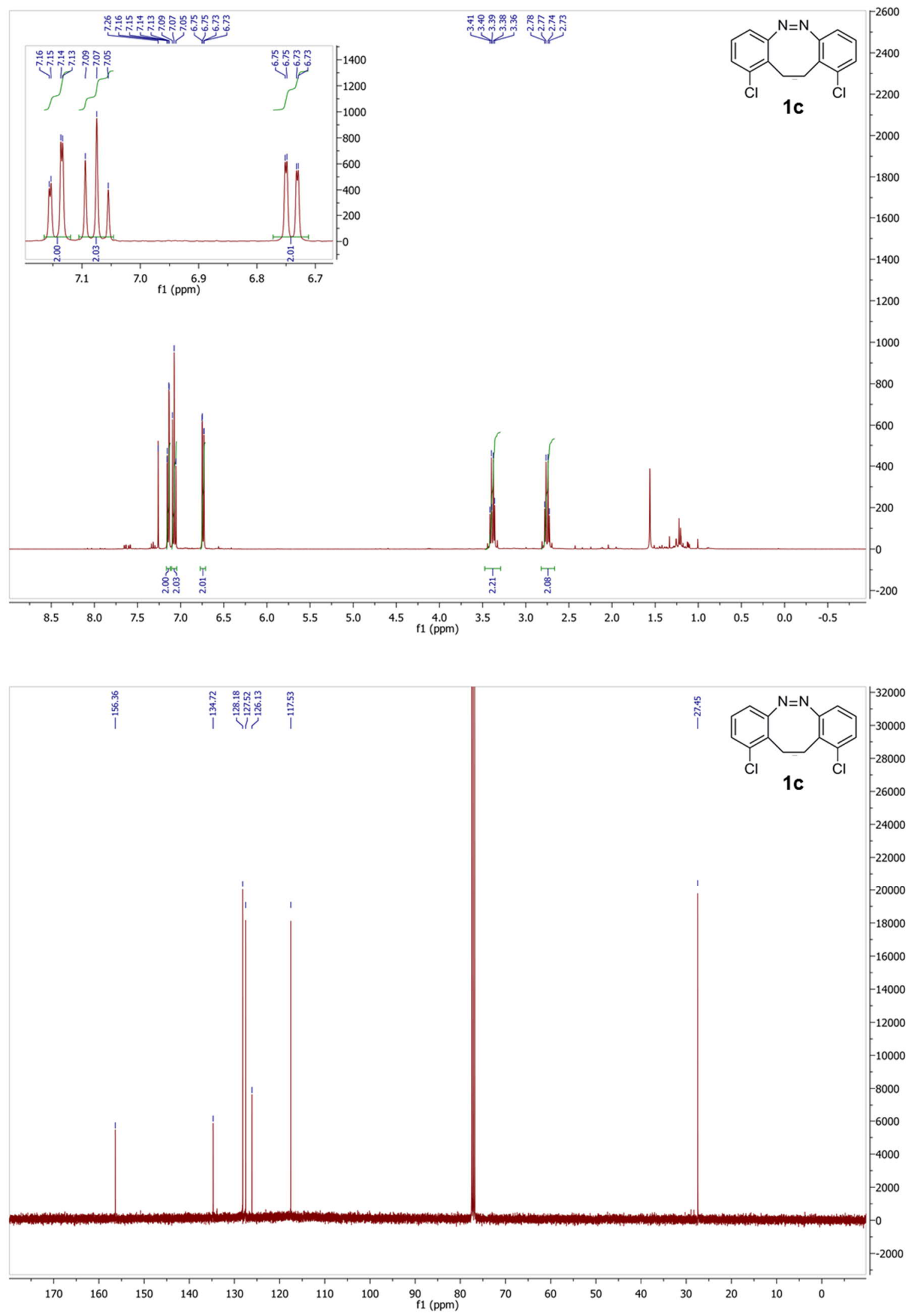

Figure S26. ${ }^{1} \mathrm{H}$ NMR (400 MHz) and ${ }^{13} \mathrm{C}$ NMR (101 MHz) spectra of 1,10-dichloro-11,12dihydrodibenzo[c,g]-1,2-diazocine $1 \mathrm{c}$ in $\mathrm{CDCl}_{3}$. 

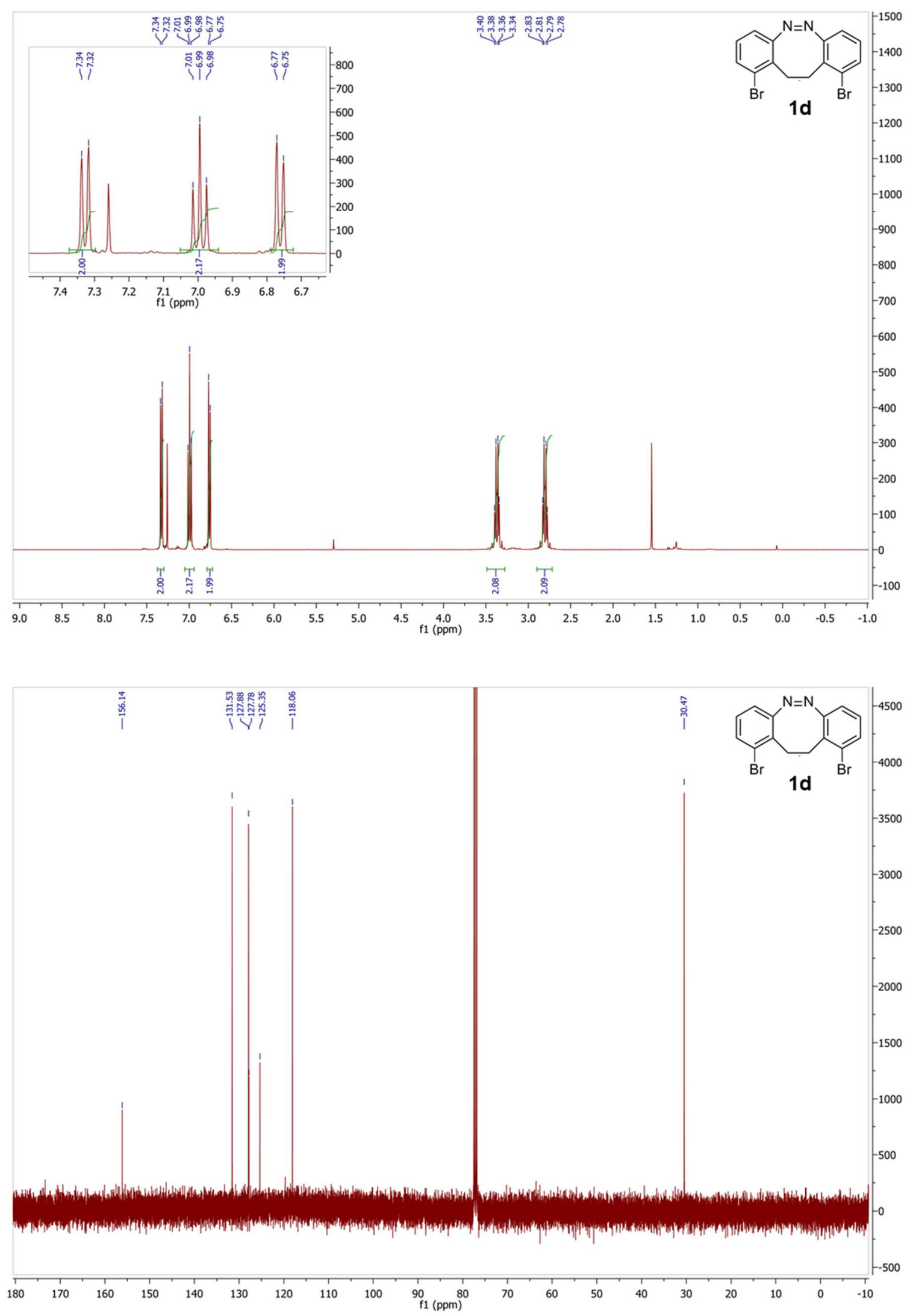

Figure S27. ${ }^{1} \mathrm{H}$ NMR $(400 \mathrm{MHz})$ and ${ }^{13} \mathrm{C}$ NMR $(101 \mathrm{MHz})$ spectra of 1,10-dibromo-11,12dihydrodibenzo[c,g]-1,2-diazocine $\mathbf{1 d}$ in $\mathrm{CDCl}_{3}$. 

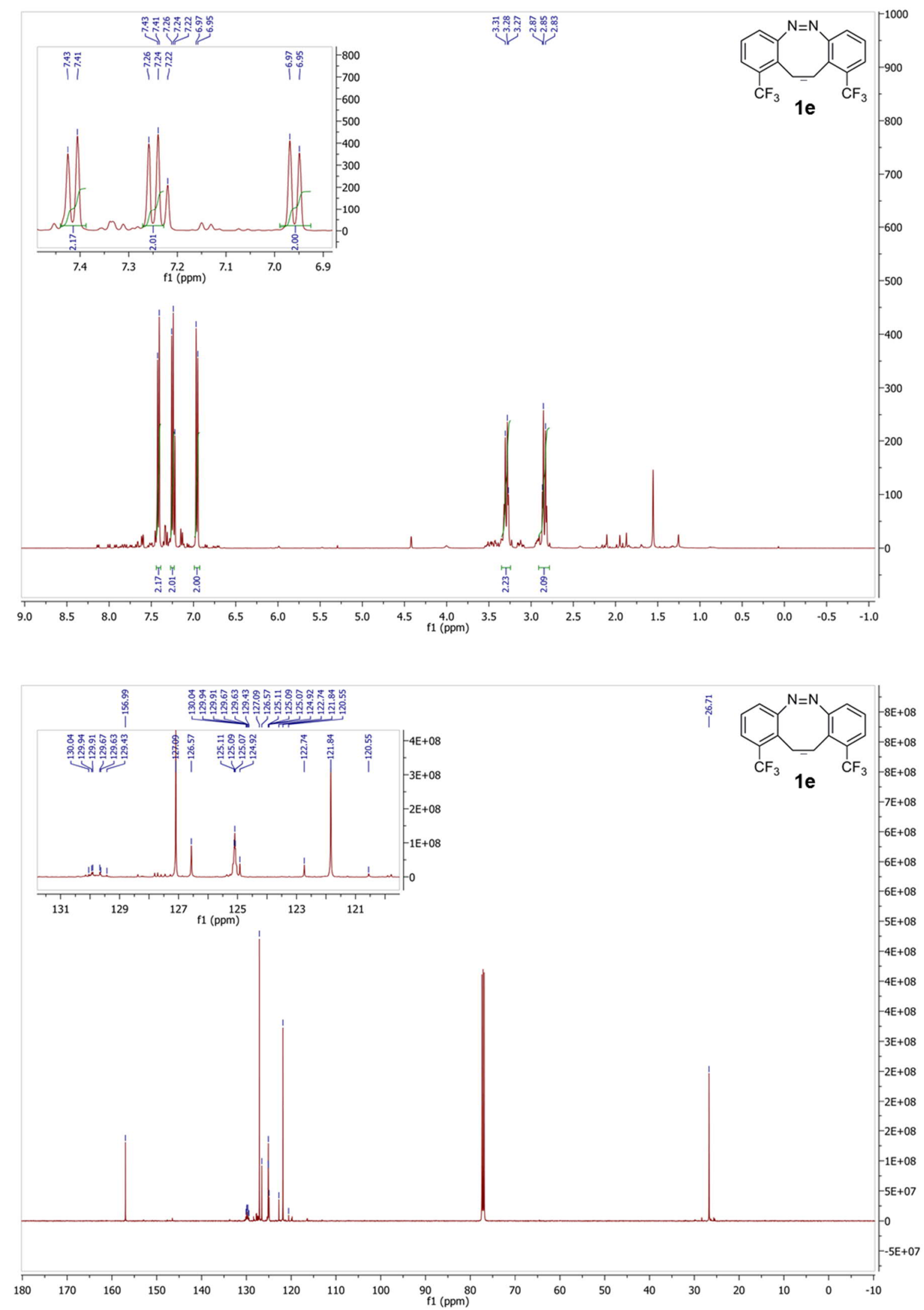

Figure S28. ${ }^{1} \mathrm{H} \quad \mathrm{NMR}(400 \mathrm{MHz})$ and ${ }^{13} \mathrm{C} \quad \mathrm{NMR}(126 \mathrm{MHz})$ spectra of $1,10-$ bis(trifluoromethyl)-11,12-dihydrodibenzo[c,g]-1,2-diazocine $1 \mathbf{e}$ in $\mathrm{CDCl}_{3}$. 

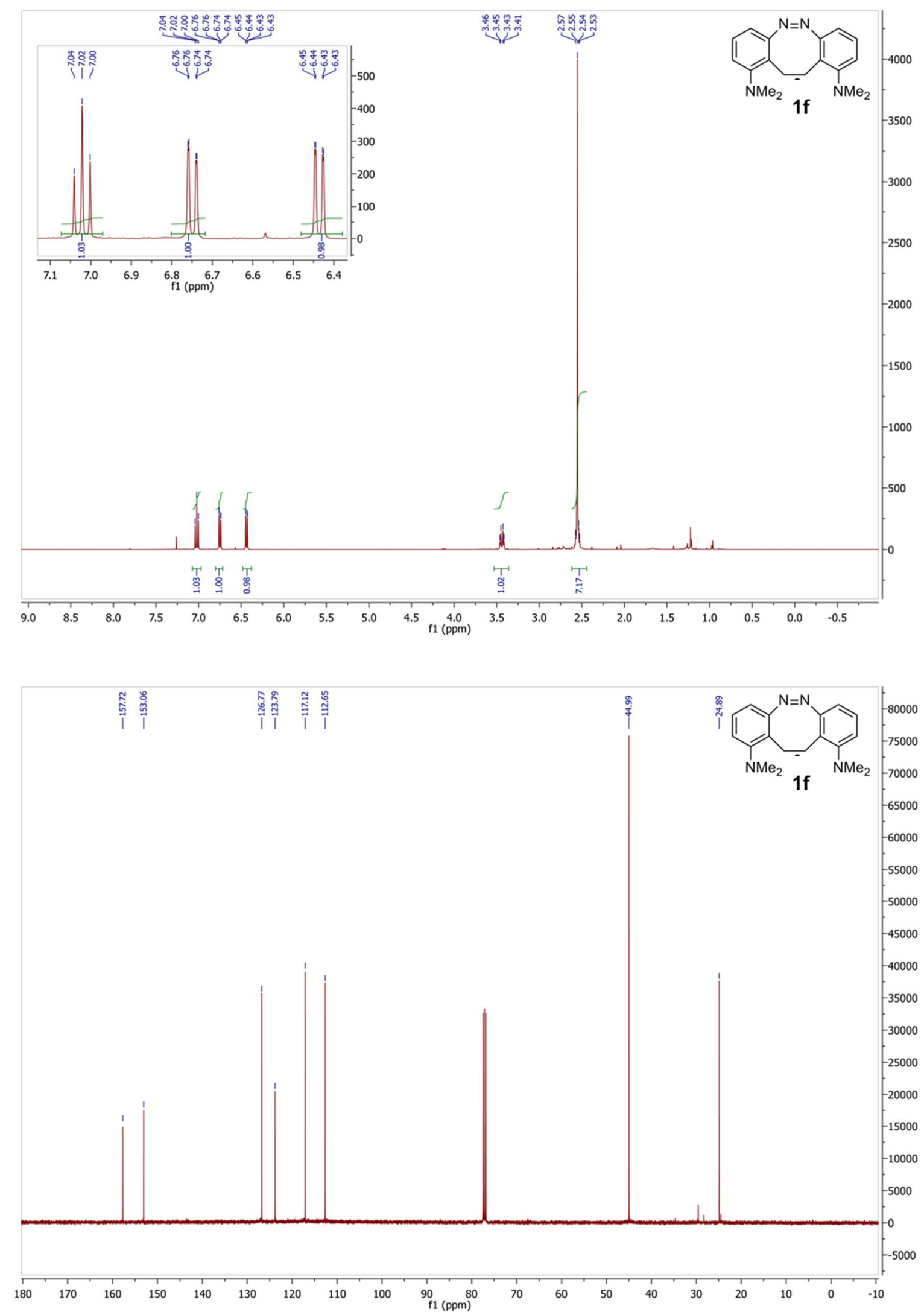

Figure S29. ${ }^{1} \mathrm{H}$ NMR (400 $\left.\mathrm{MHz}\right)$ and ${ }^{13} \mathrm{C} \quad \mathrm{NMR}(101 \mathrm{MHz})$ spectra of $1,10-$ bis(dimethylamino)-11,12-dihydrodibenzo[c,g]-1,2-diazocine $\mathbf{1 f}$ in $\mathrm{CDCl}_{3}$. 

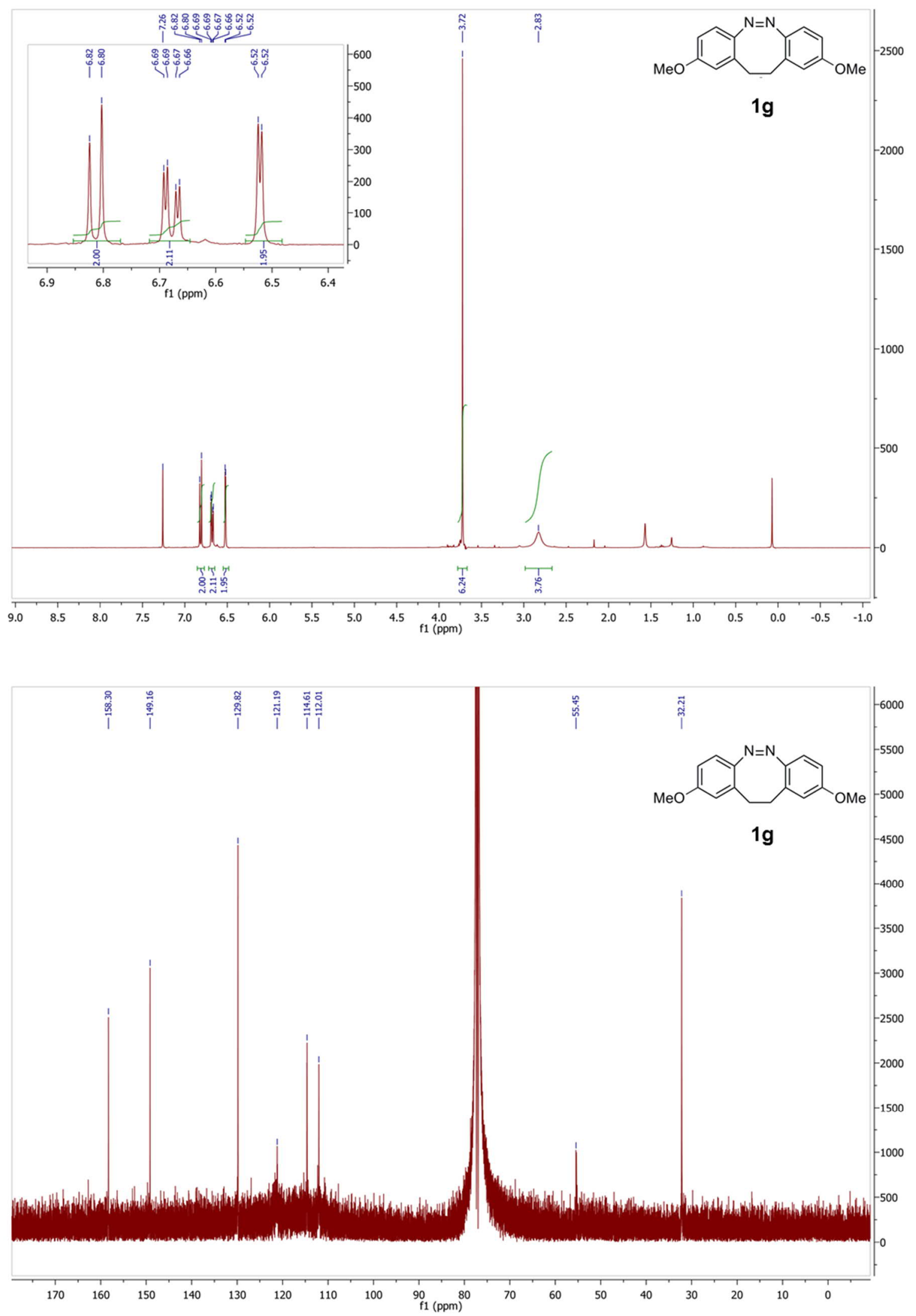

Figure S30. ${ }^{1} \mathrm{H}$ NMR (400 MHz) and ${ }^{13} \mathrm{C}$ NMR (101 MHz) spectra of 2,9-dimethoxy-11,12dihydrodibenzo[c,g]-1,2-diazocine $\mathbf{1 g}$ in $\mathrm{CDCl}_{3}$. 

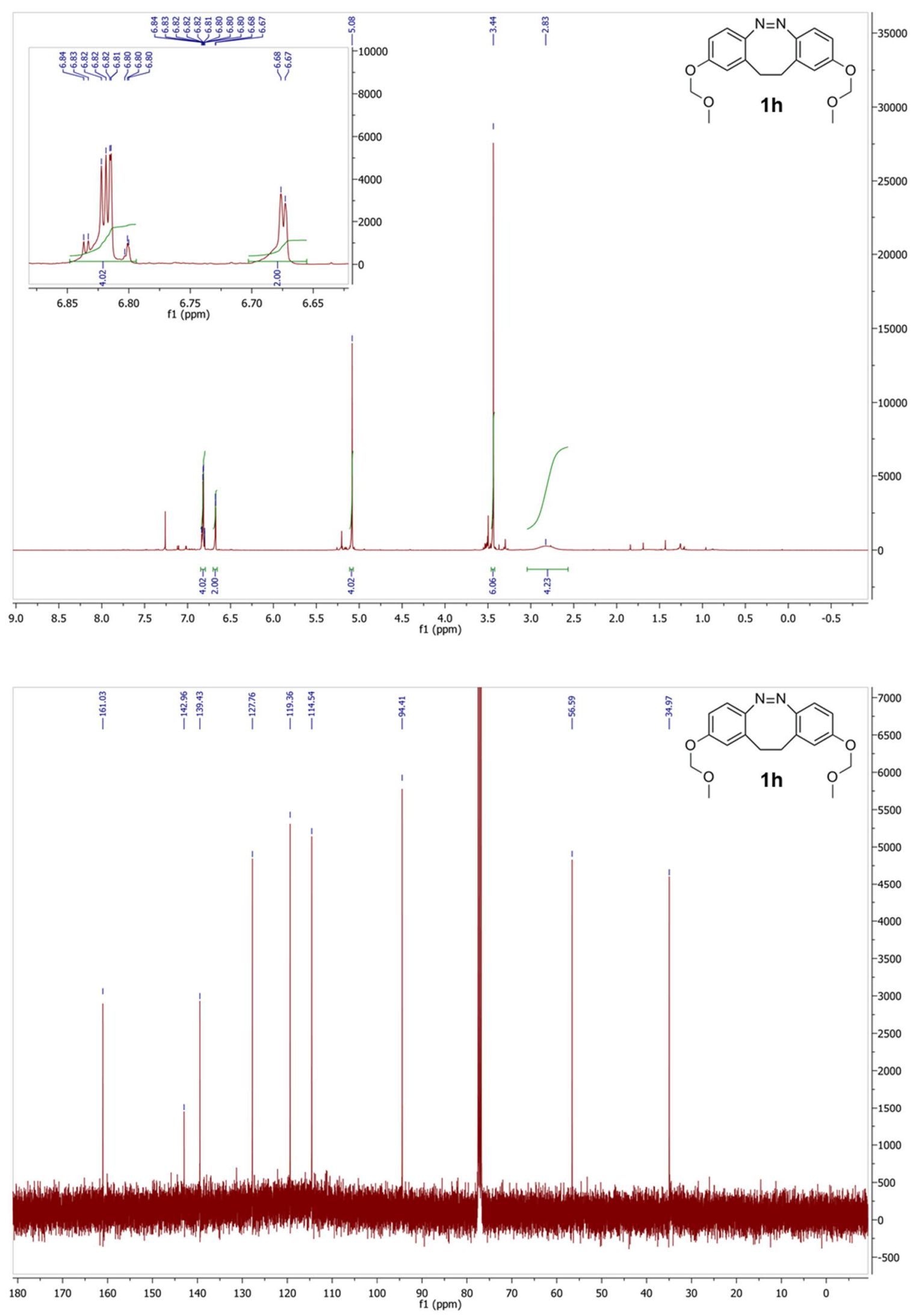

Figure S31. ${ }^{1} \mathrm{H} \quad \mathrm{NMR} \quad(600 \mathrm{MHz})$ and ${ }^{13} \mathrm{C} \quad \mathrm{NMR} \quad(151 \mathrm{MHz})$ spectra of 2,9bis(methoxymethoxy)-11,12-dihydrodibenzo[c,g]-1,2-diazocine $\mathbf{1 h}$ in $\mathrm{CDCl}_{3}$. 

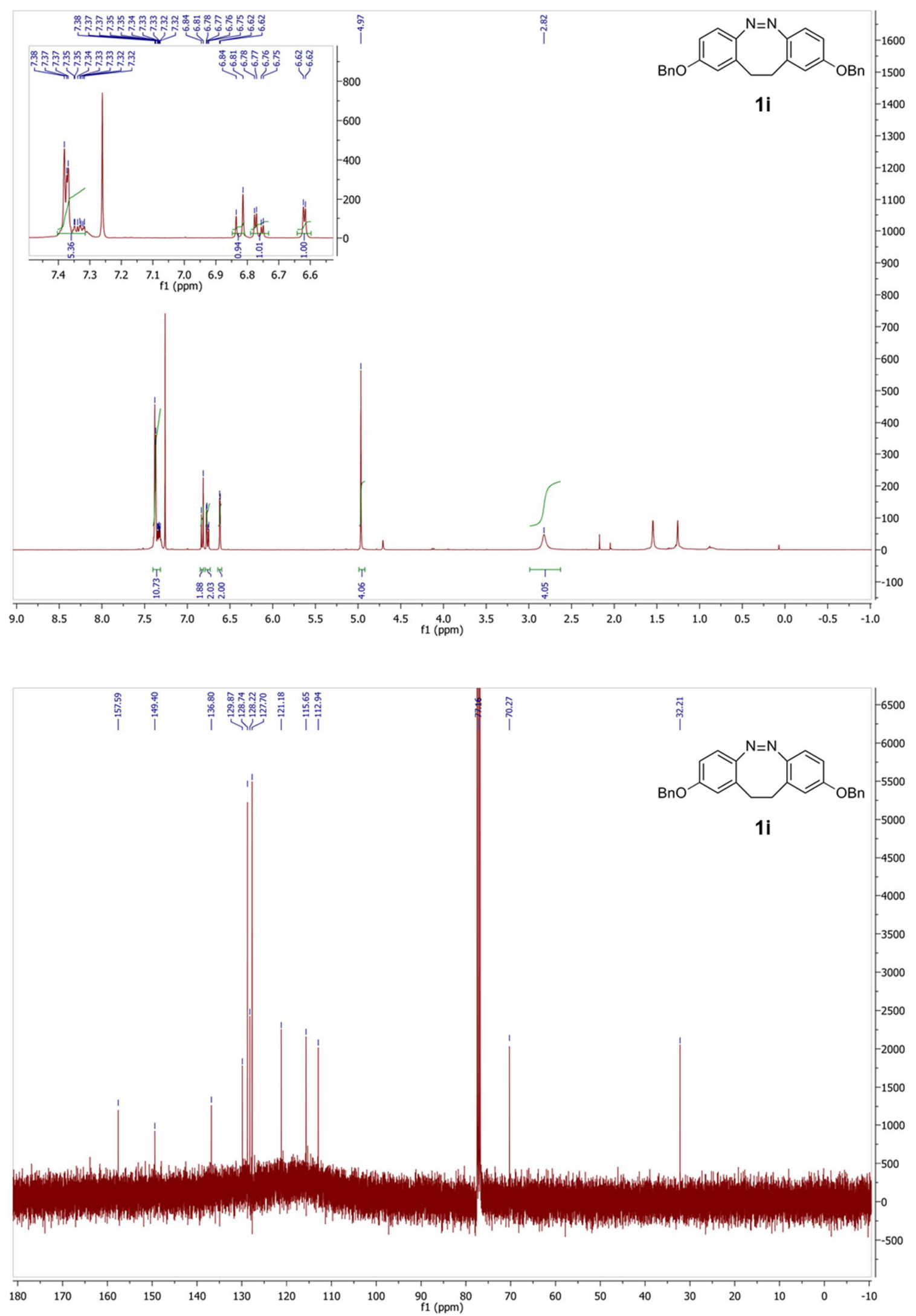

Figure S32. ${ }^{1} \mathrm{H}$ NMR (400 MHz) and ${ }^{13} \mathrm{C}$ NMR (101 MHz) spectra of 2,9-dibenzyloxy-11,12dihydrodibenzo[ $c, g]$-1,2-diazocine $\mathbf{1 i}$ in $\mathrm{CDCl}_{3}$. 

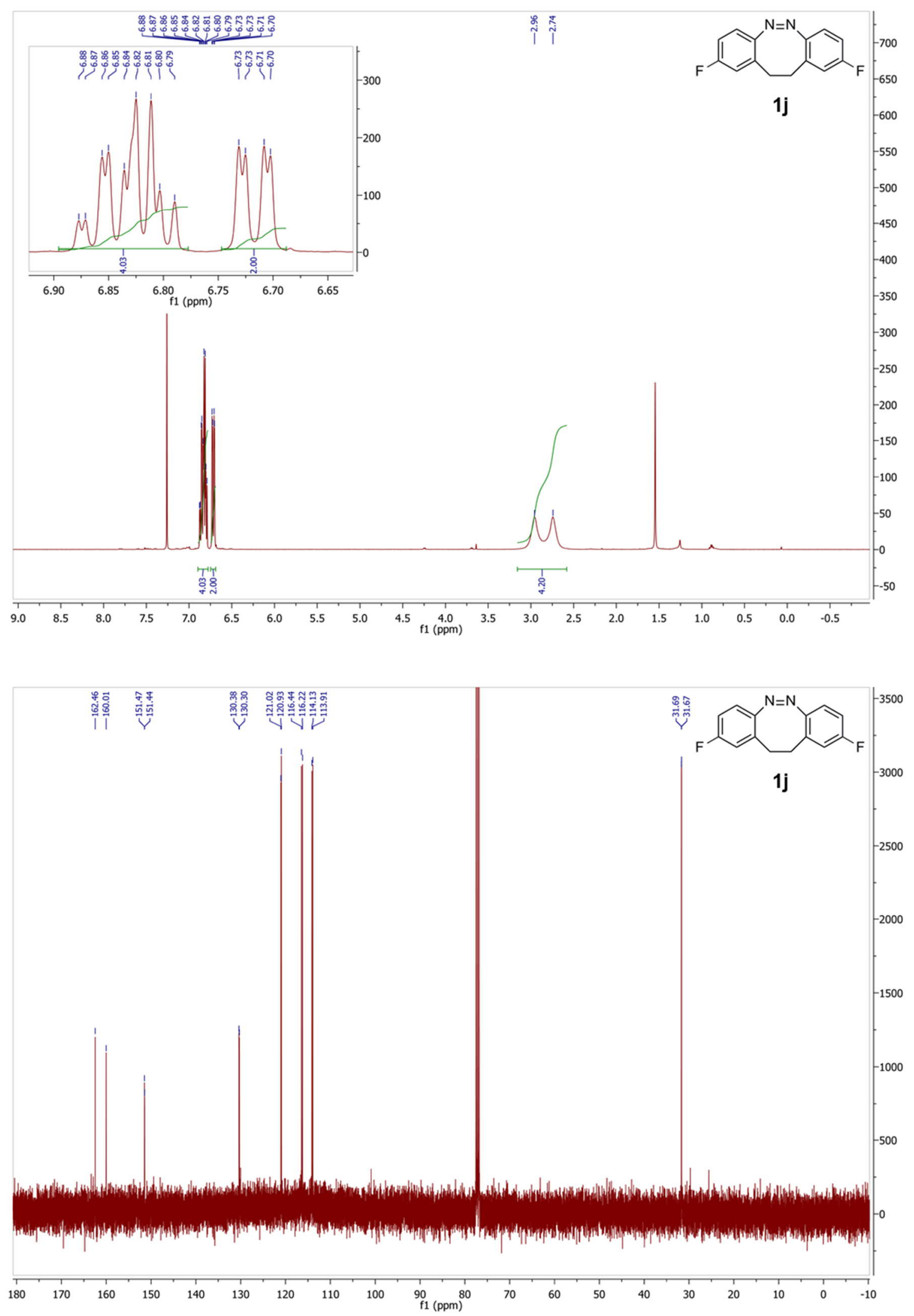

Figure S33. ${ }^{1} \mathrm{H}$ NMR (400 MHz) and ${ }^{13} \mathrm{C}$ NMR (101 MHz) spectra of 2,9-difluoro-11,12dihydrodibenzo[ $[c, g]$-1,2-diazocine $\mathbf{1} \mathbf{j}$ in $\mathrm{CDCl}_{3}$. 

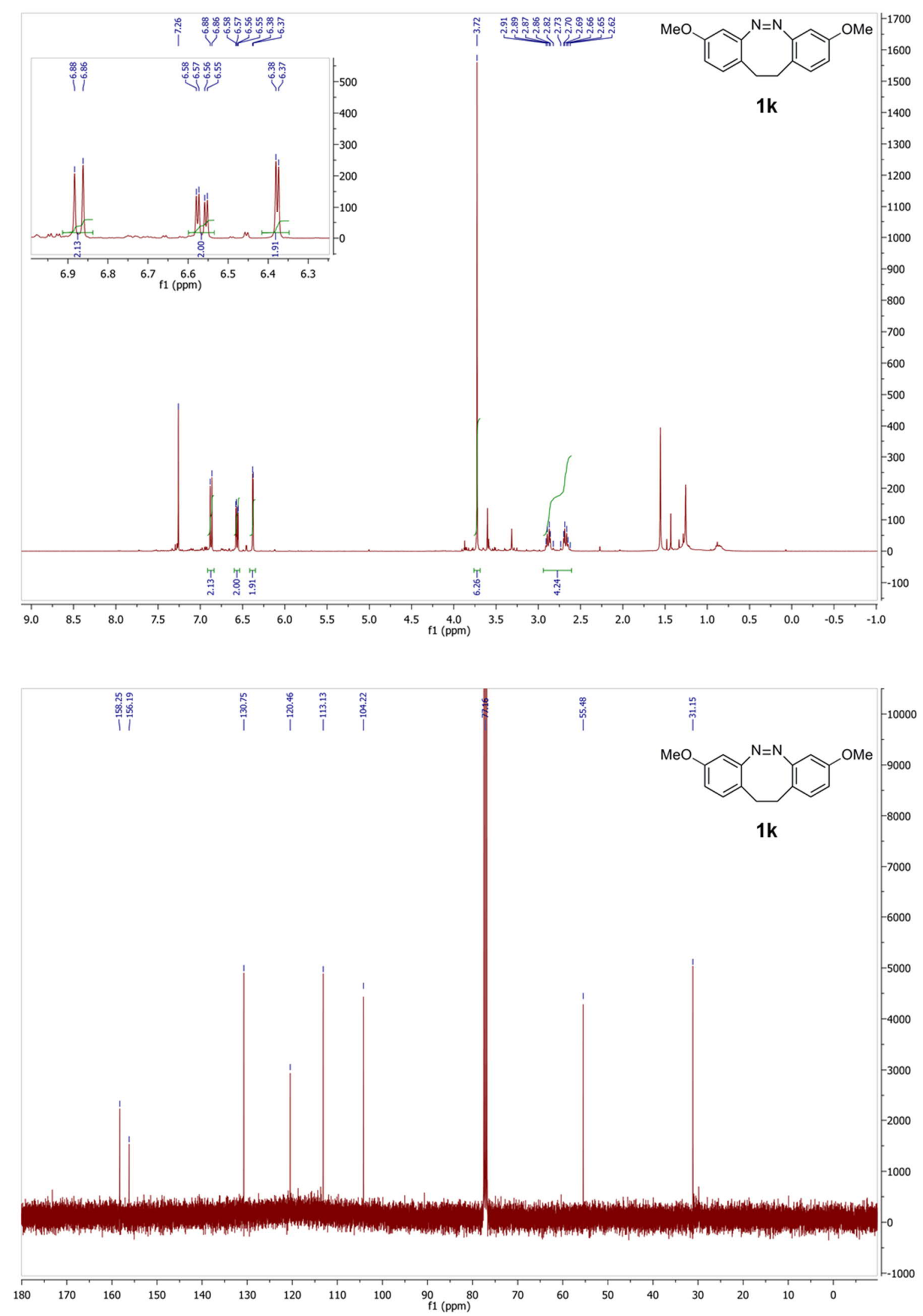

Figure S34. ${ }^{1} \mathrm{H}$ NMR (400 MHz) and ${ }^{13} \mathrm{C}$ NMR (101 MHz) spectra of 3,8-dimethoxy-11,12dihydrodibenzo[ $[c, g]$-1,2-diazocine $\mathbf{1 k}$ in $\mathrm{CDCl}_{3}$. 

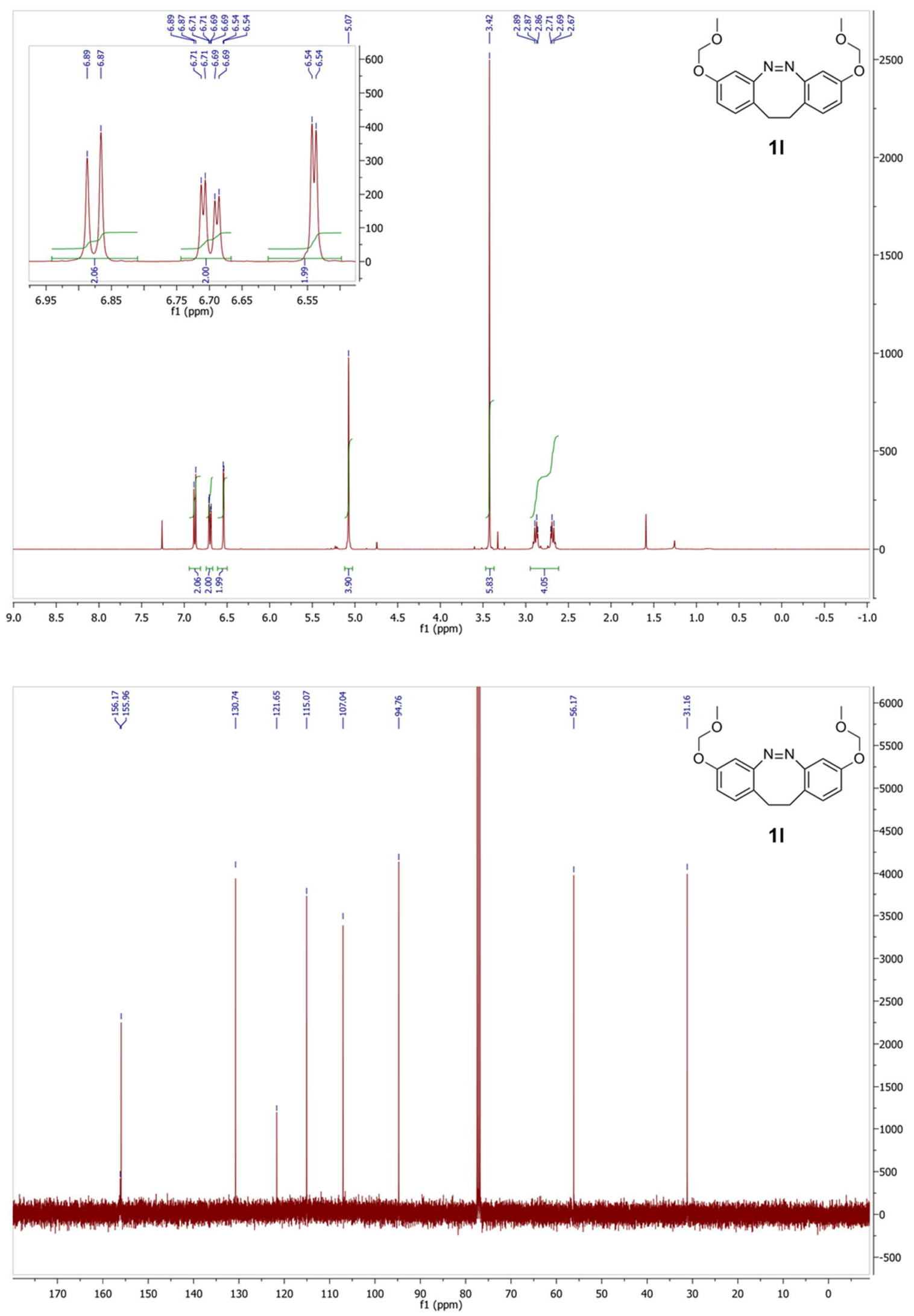

Figure S35. ${ }^{1} \mathrm{H} \quad \mathrm{NMR} \quad(400 \mathrm{MHz})$ and ${ }^{13} \mathrm{C} \quad \mathrm{NMR} \quad(101 \mathrm{MHz})$ spectra of 3,8bis(methoxymethoxy)-11,12-dihydrodibenzo[c,g]-1,2-diazocine 11 in $\mathrm{CDCl}_{3}$. 

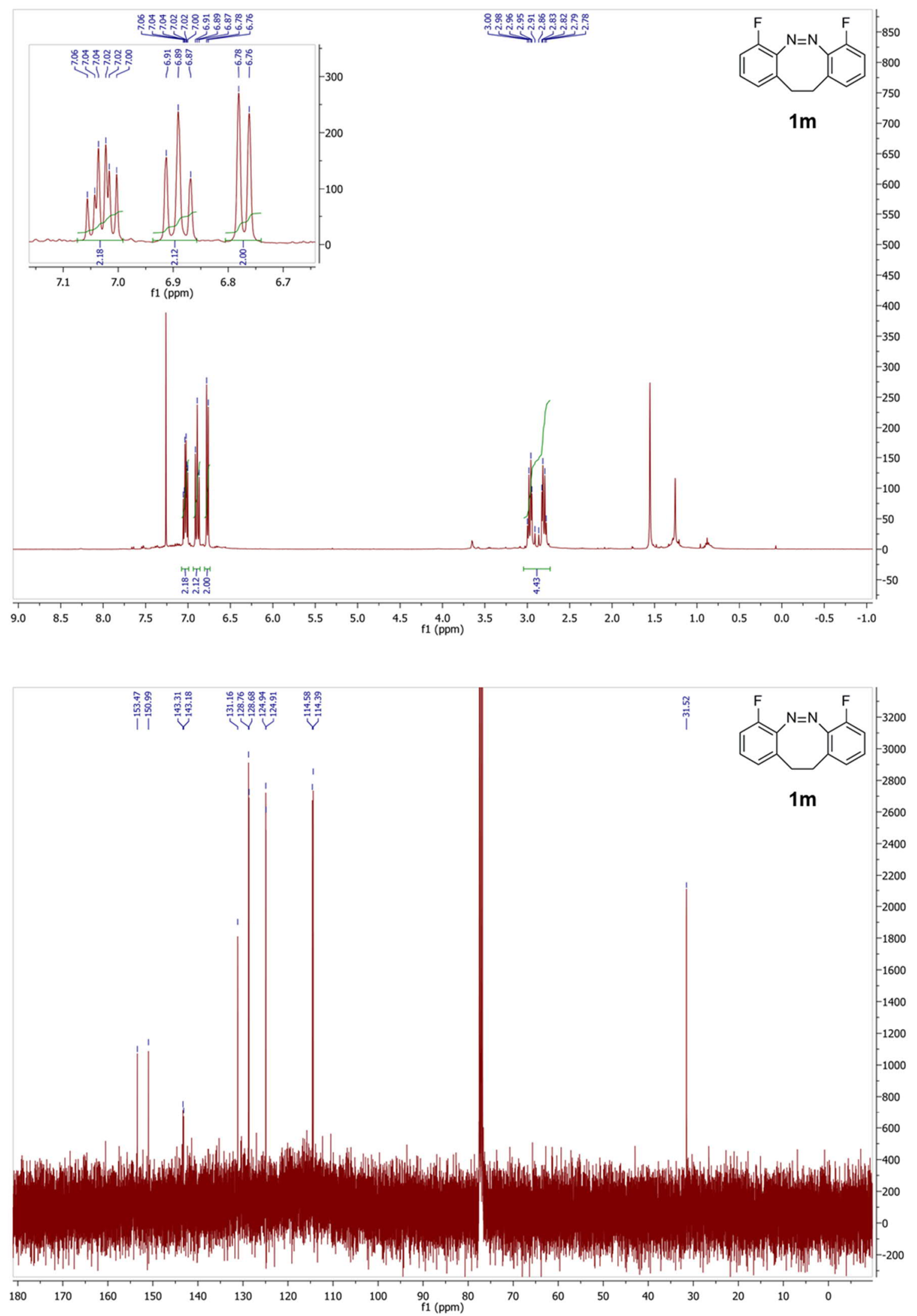

Figure S36. ${ }^{1} \mathrm{H}$ NMR (400 MHz) and ${ }^{13} \mathrm{C}$ NMR (101 MHz) spectra of 4,7-difluoro-11,12dihydrodibenzo[c,g]-1,2-diazocine $\mathbf{1 m}$ in $\mathrm{CDCl}_{3}$. 


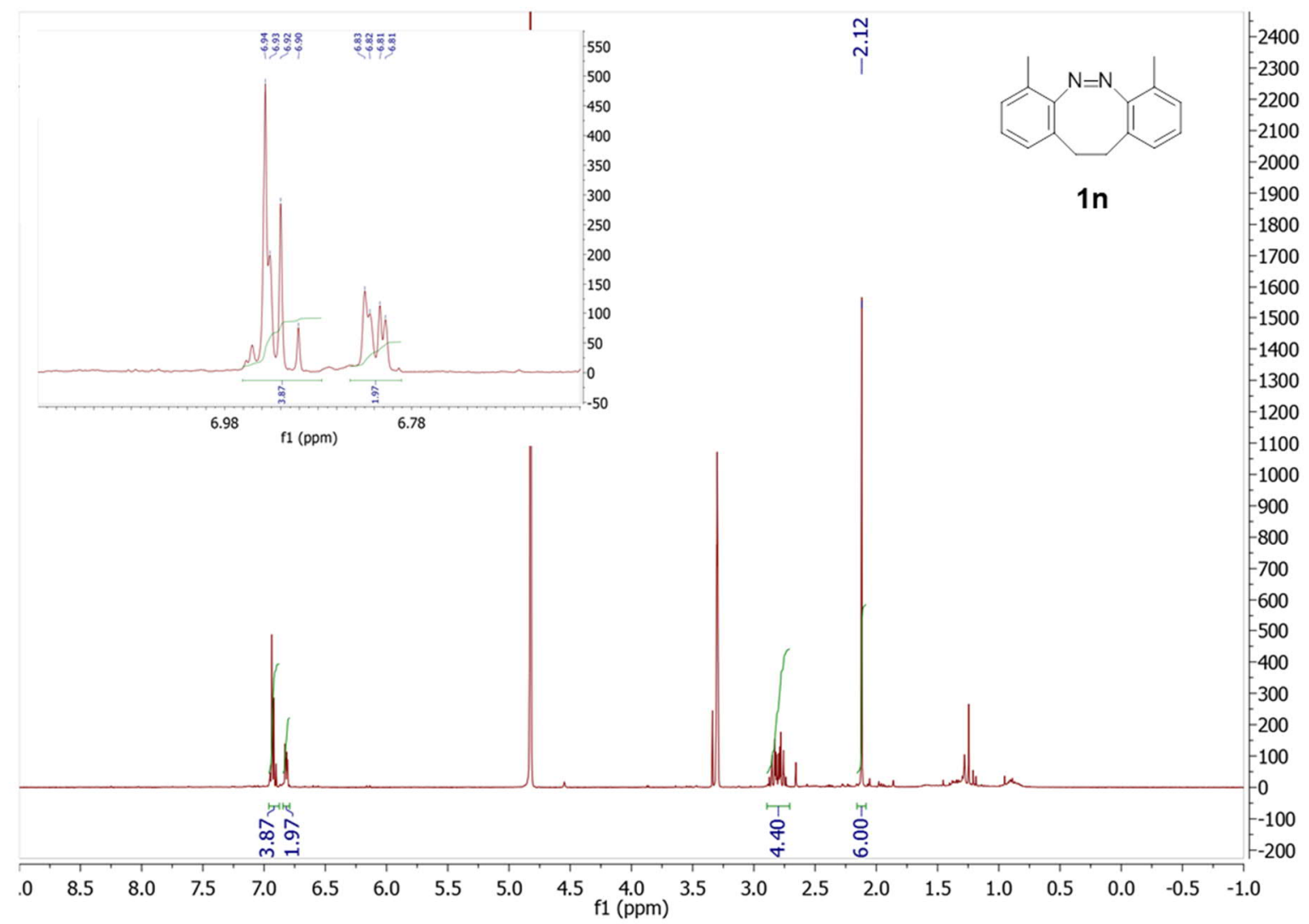

Figure S37. ${ }^{1} \mathrm{H}$ NMR (400 MHz) spectrum of 4,7-dimethyl-11,12-dihydrodibenzo[c,g]-1,2diazocine $1 \mathrm{n}$ in $\mathrm{CDCl}_{3}$. 

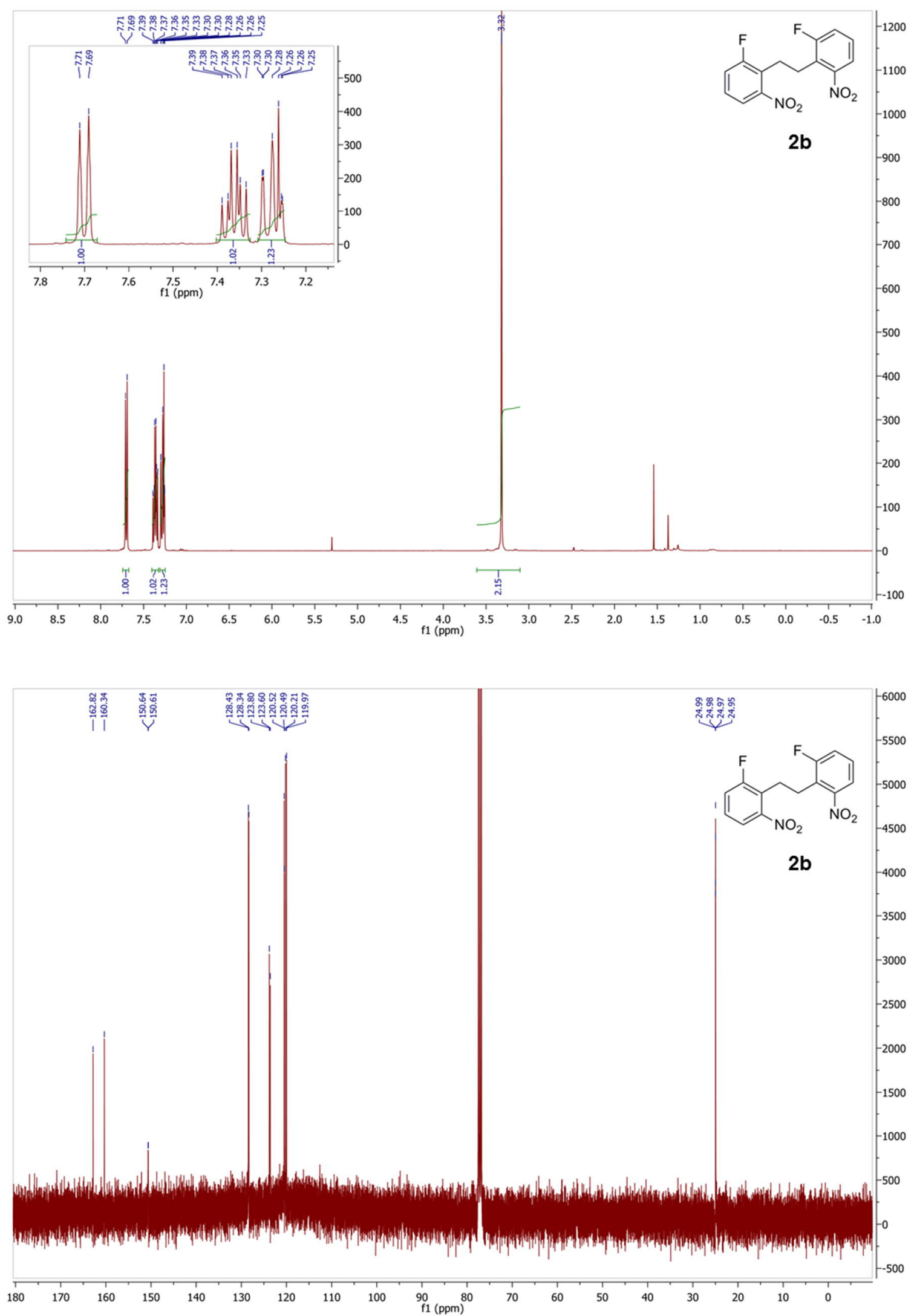

Figure S38. ${ }^{1} \mathrm{H}$ NMR (400 MHz) and ${ }^{13} \mathrm{C}$ NMR (101 MHz) spectra of 1,2-bis(2-fluoro-6nitrophenyl)ethane $\mathbf{2 b}$ in $\mathrm{CDCl}_{3}$. 

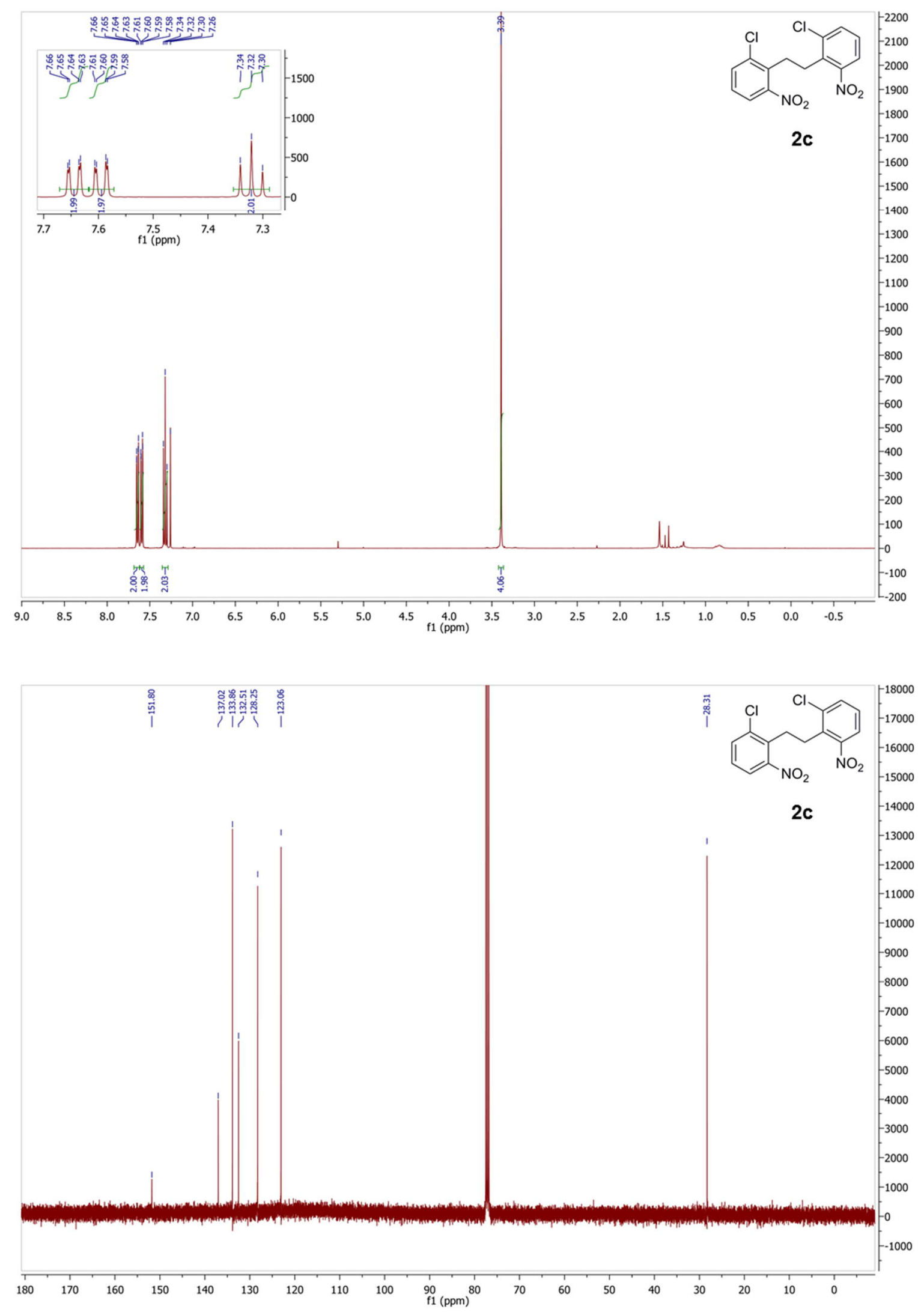

Figure S39. ${ }^{1} \mathrm{H}$ NMR (400 MHz) and ${ }^{13} \mathrm{C}$ NMR (101 MHz) spectra of 1,2-bis(2-chloro-6nitrophenyl)ethane $2 \mathrm{c}$ in $\mathrm{CDCl}_{3}$. 

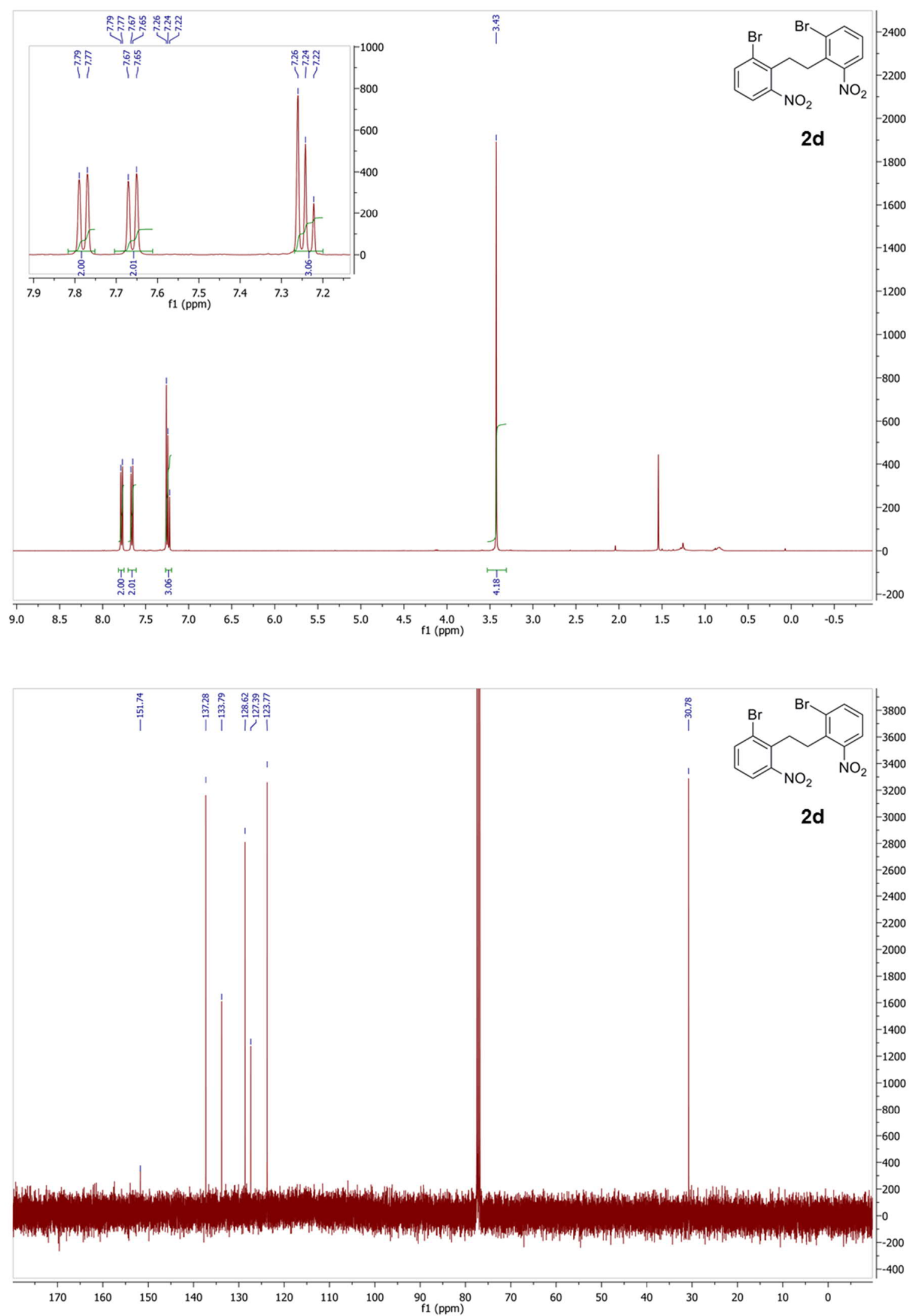

Figure S40. ${ }^{1} \mathrm{H}$ NMR (400 MHz) and ${ }^{13} \mathrm{C}$ NMR (101 MHz) spectra of 1,2-bis(2-bromo-6nitrophenyl)ethane $\mathbf{2 d}$ in $\mathrm{CDCl}_{3}$. 

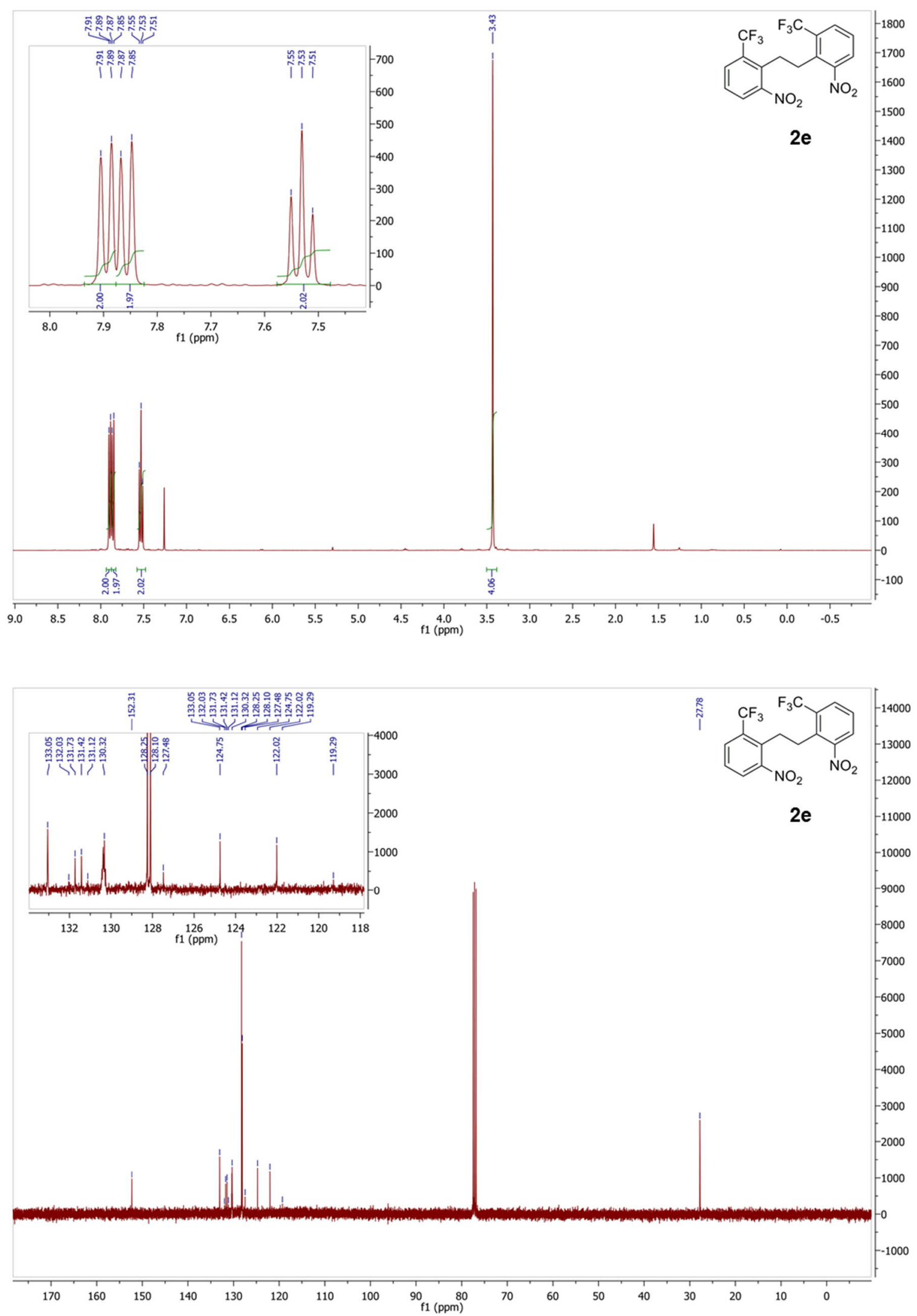

Figure S41. ${ }^{1} \mathrm{H}$ NMR (400 $\left.\mathrm{MHz}\right)$ and ${ }^{13} \mathrm{C}$ NMR (101 $\left.\mathrm{MHz}\right)$ spectra of 1,2-bis(6trifluoromethyl-2-nitrophenyl)ethane $\mathbf{2 e}$ in $\mathrm{CDCl}_{3}$. 

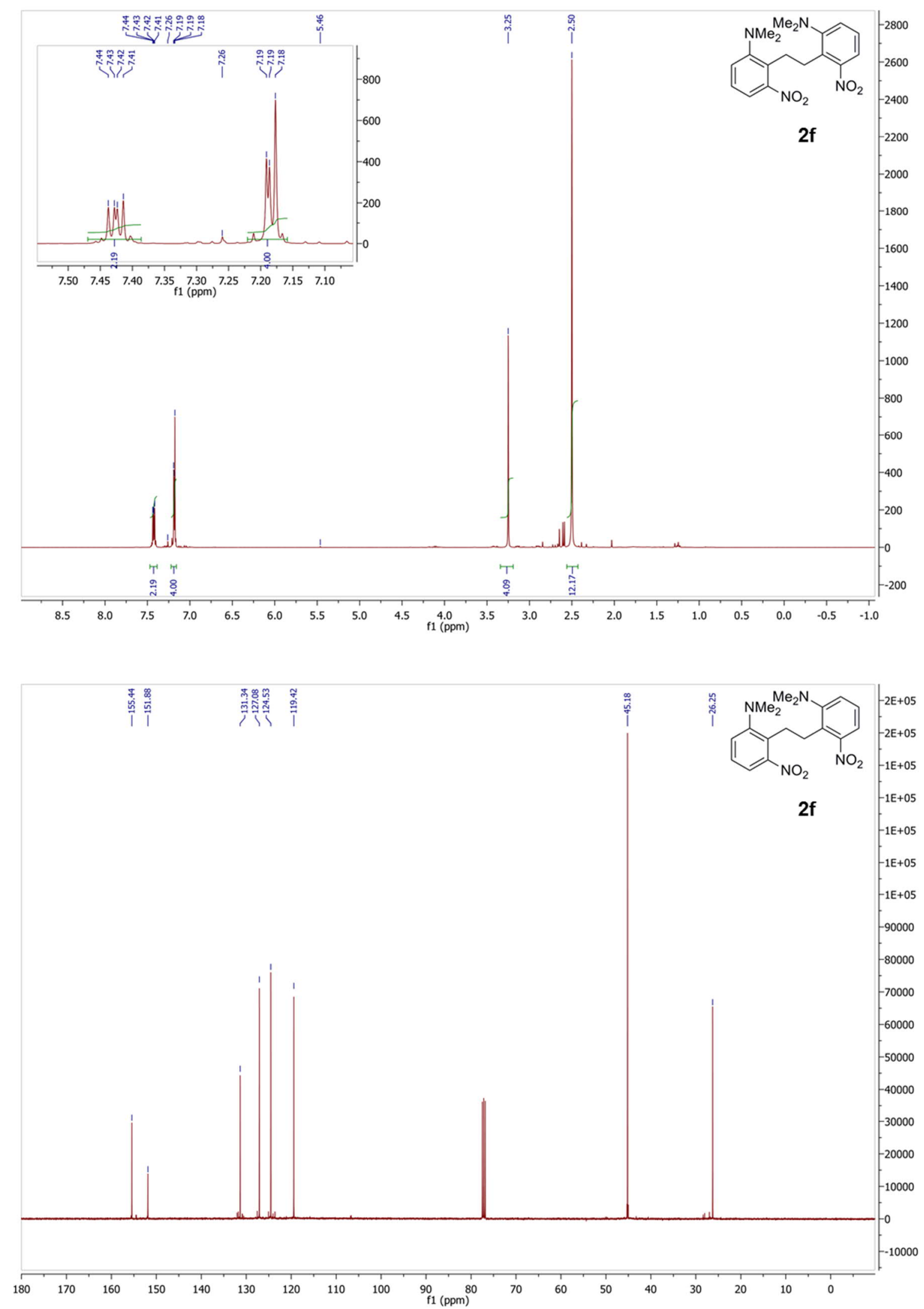

Figure S42. ${ }^{1} \mathrm{H}$ NMR (400 $\left.\mathrm{MHz}\right)$ and ${ }^{13} \mathrm{C}$ NMR (101 $\left.\mathrm{MHz}\right)$ spectra of 1,2-bis(2(dimethylamino)-6-nitrophenyl)ethane $\mathbf{2 f}$ in $\mathrm{CDCl}_{3}$. 

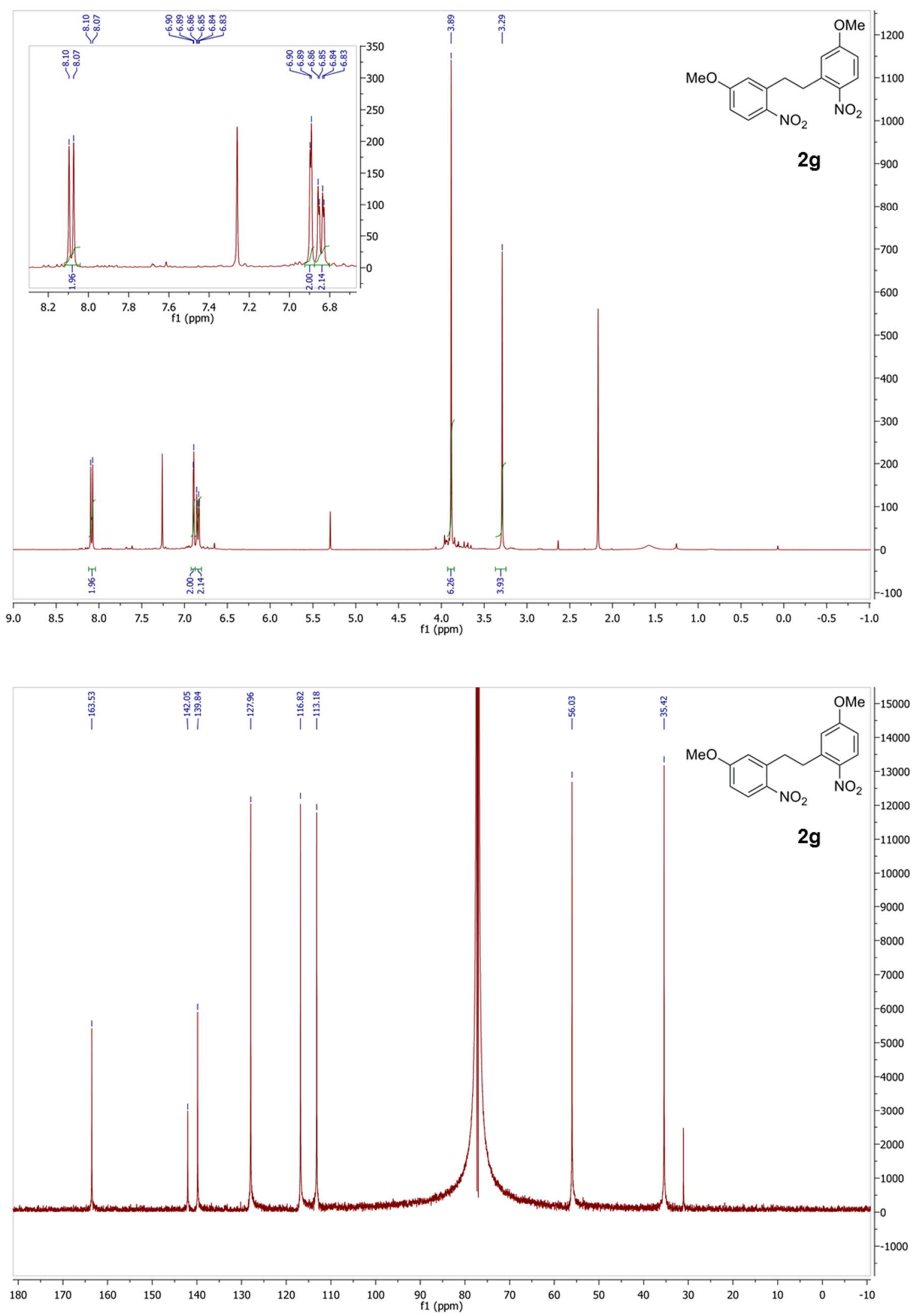

Figure S43. ${ }^{1} \mathrm{H}$ NMR (400 MHz) and ${ }^{13} \mathrm{C}$ NMR (126 MHz) spectra of 1,2-bis(5-methoxy-2nitrophenyl)ethane $\mathbf{2 g}$ in $\mathrm{CDCl}_{3}$. 

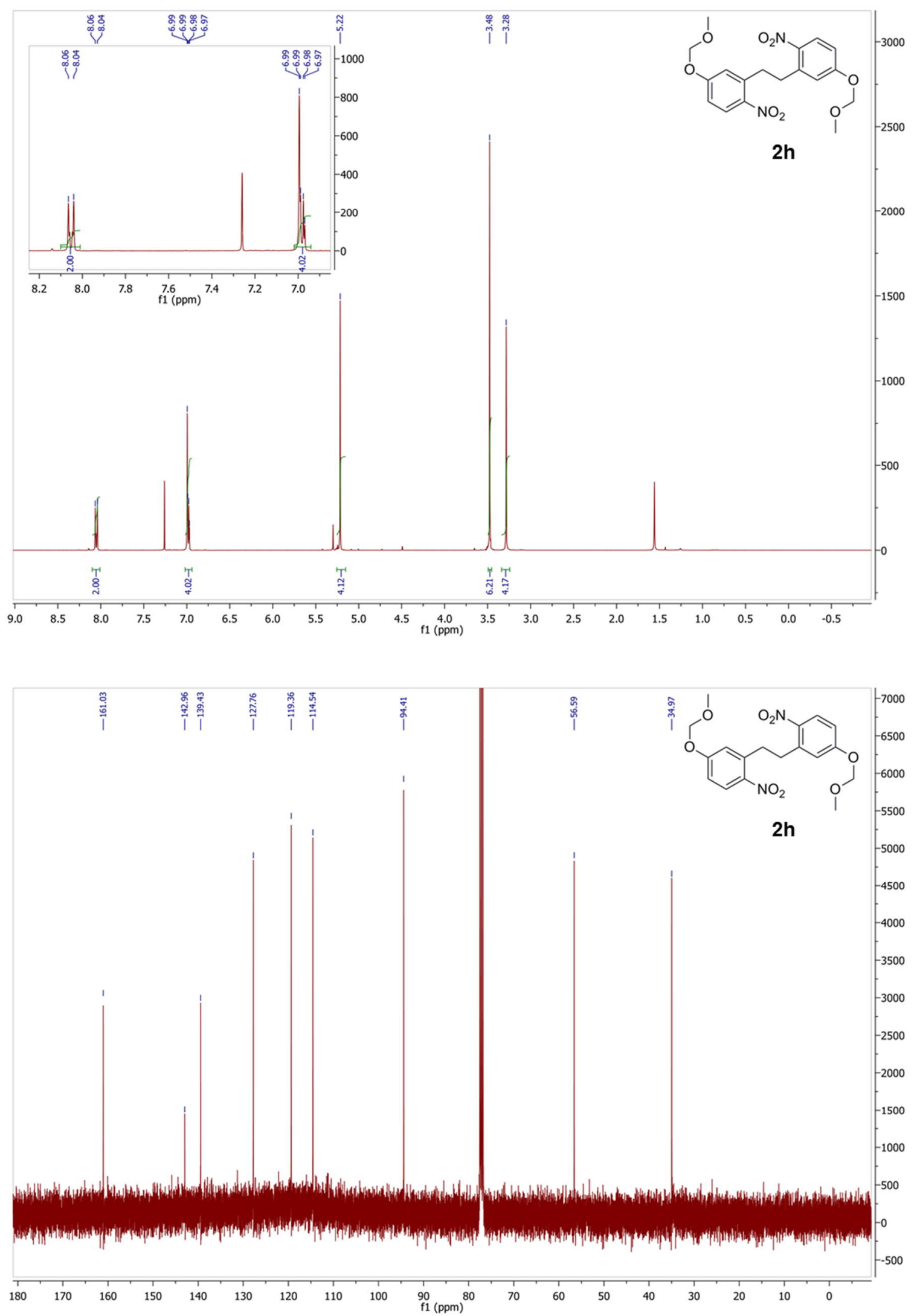

Figure S44. ${ }^{1} \mathrm{H}$ NMR (400 $\left.\mathrm{MHz}\right)$ and ${ }^{13} \mathrm{C}$ NMR (101 $\left.\mathrm{MHz}\right)$ spectra of 1,2-bis(5methoxymethoxy-2-nitrophenyl)ethane $\mathbf{2 h}$ in $\mathrm{CDCl}_{3}$. 

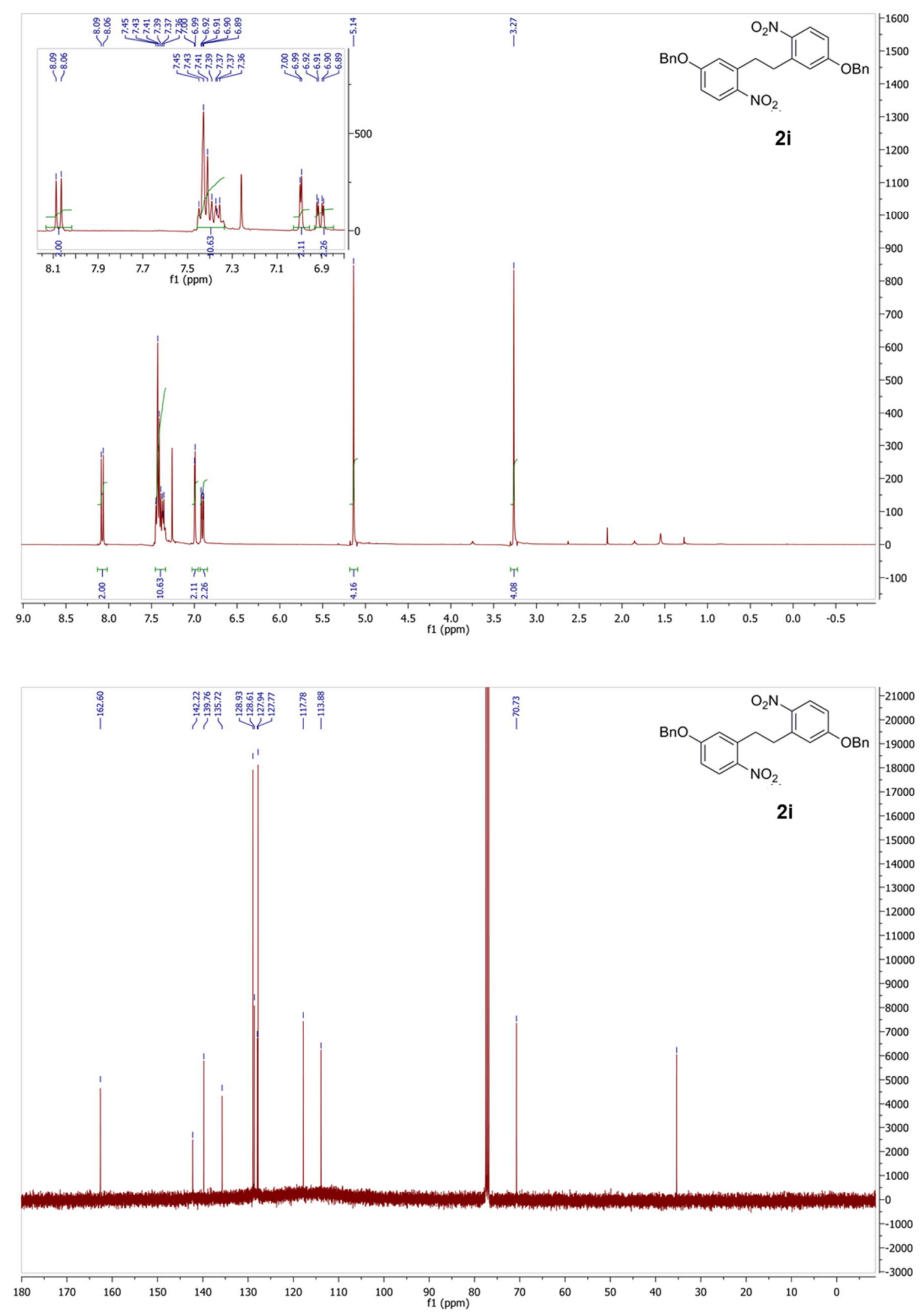

Figure S45. ${ }^{1} \mathrm{H}$ NMR (400 MHz) and ${ }^{13} \mathrm{C}$ NMR (101 MHz) spectra of 1,2-bis(5-benzyloxy-2nitrophenyl)ethane $2 \mathbf{i}$ in $\mathrm{CDCl}_{3}$. 

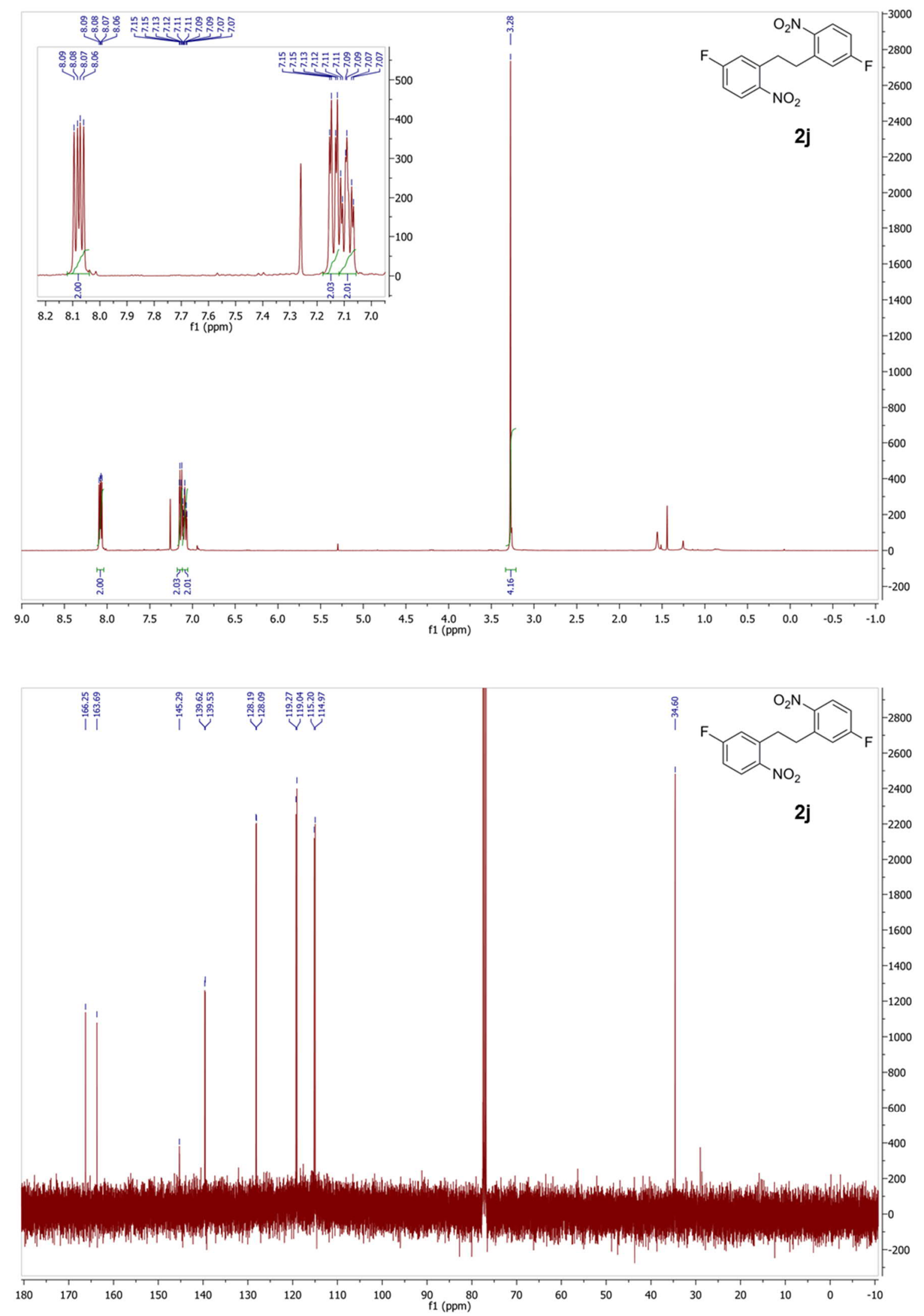

Figure S46. ${ }^{1} \mathrm{H}$ NMR (400 MHz) and ${ }^{13} \mathrm{C}$ NMR (101 MHz) spectra of 1,2-bis(3-fluoro-6nitrophenyl)ethane $\mathbf{2} \mathbf{j}$ in $\mathrm{CDCl}_{3}$. 

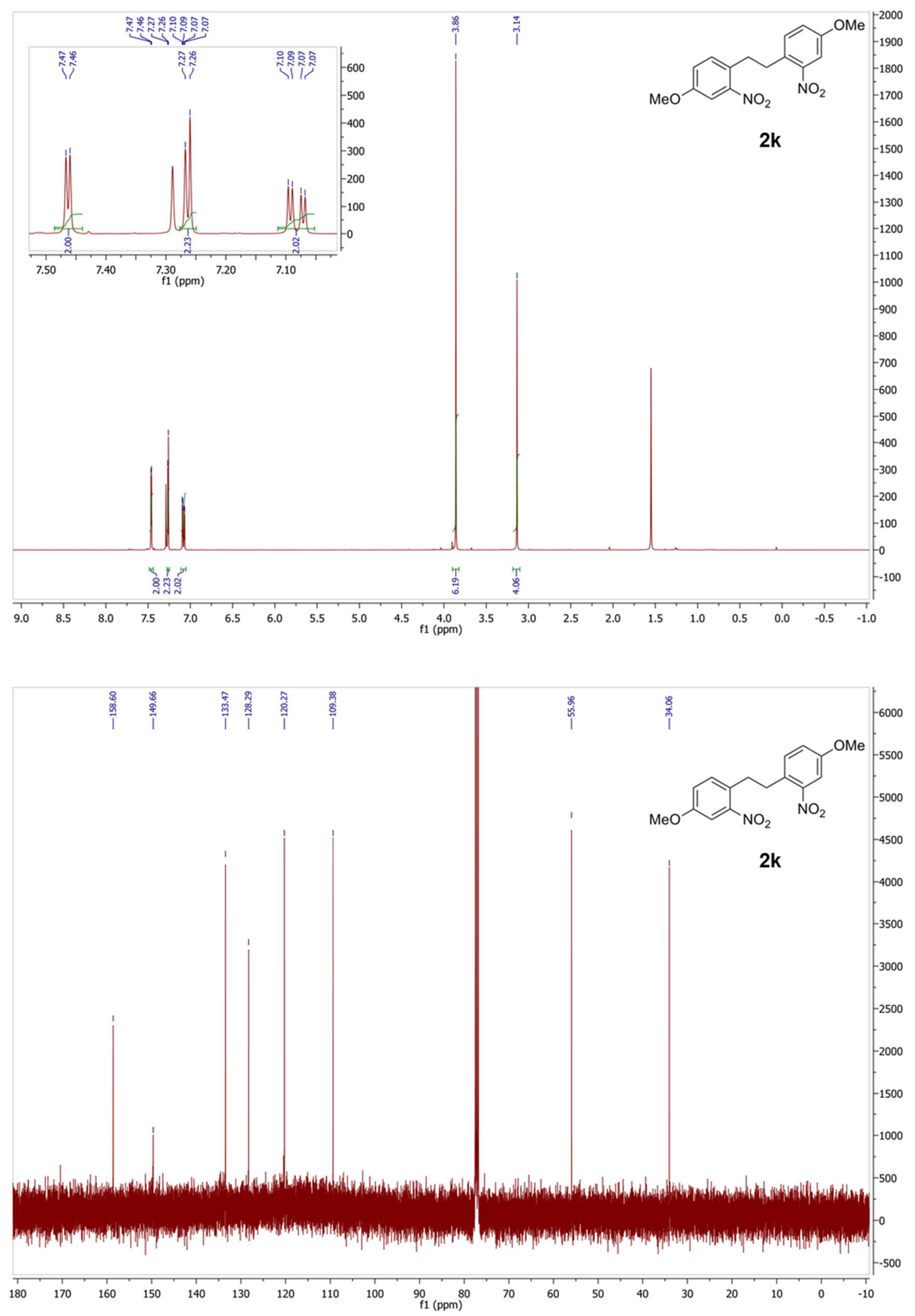

Figure S47. ${ }^{1} \mathrm{H}$ NMR (400 MHz) and ${ }^{13} \mathrm{C}$ NMR (101 MHz) spectra of 1,2-bis(4-methoxy-2nitrophenyl)ethane $\mathbf{2 k}$ in $\mathrm{CDCl}_{3}$. 

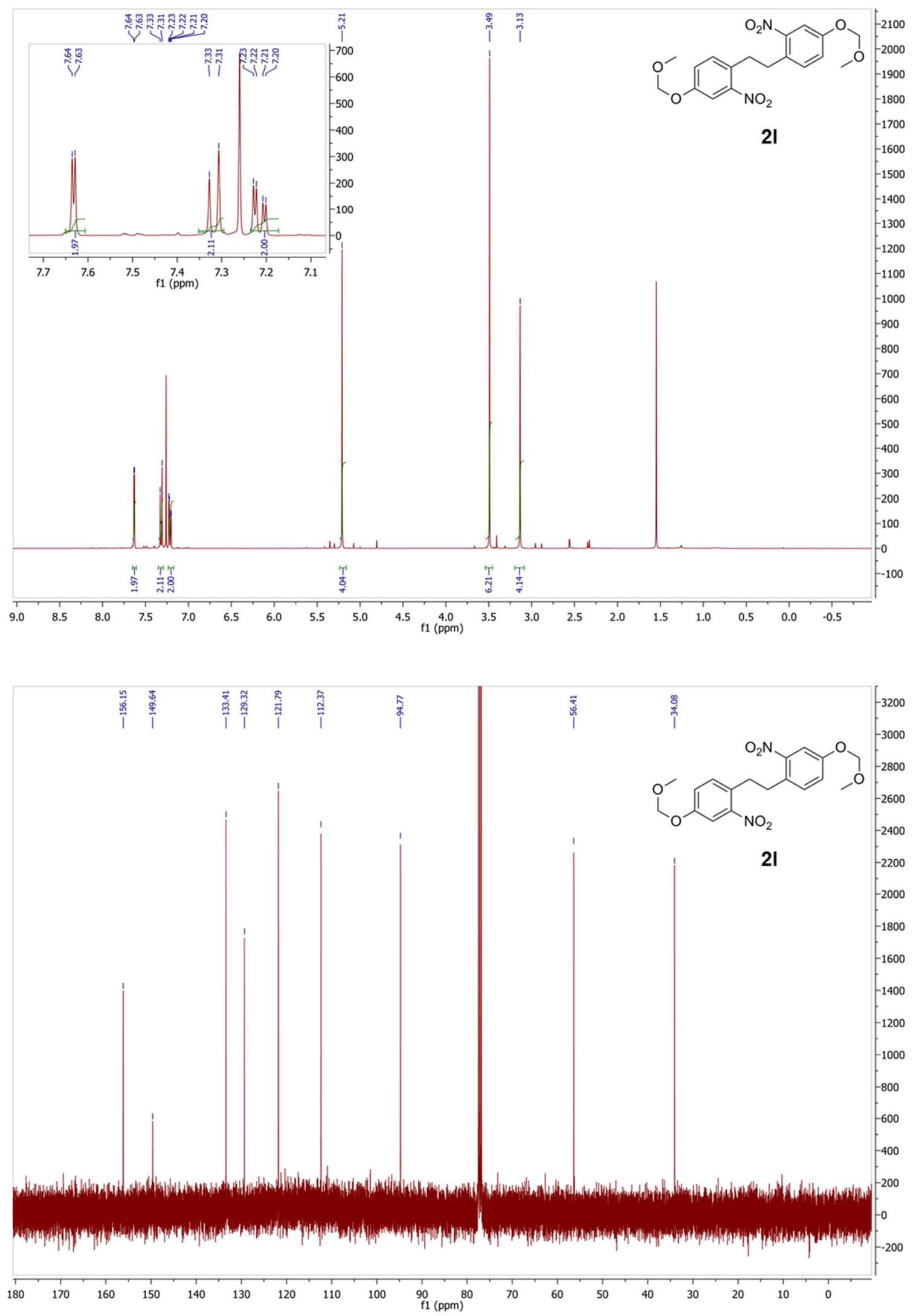

Figure S48. ${ }^{1} \mathrm{H}$ NMR (400 $\left.\mathrm{MHz}\right)$ and ${ }^{13} \mathrm{C}$ NMR (101 $\left.\mathrm{MHz}\right)$ spectra of 1,2-bis(4(methoxymethoxy)-2-nitrophenyl)ethane $2 \mathbf{l}$ in $\mathrm{CDCl}_{3}$. 

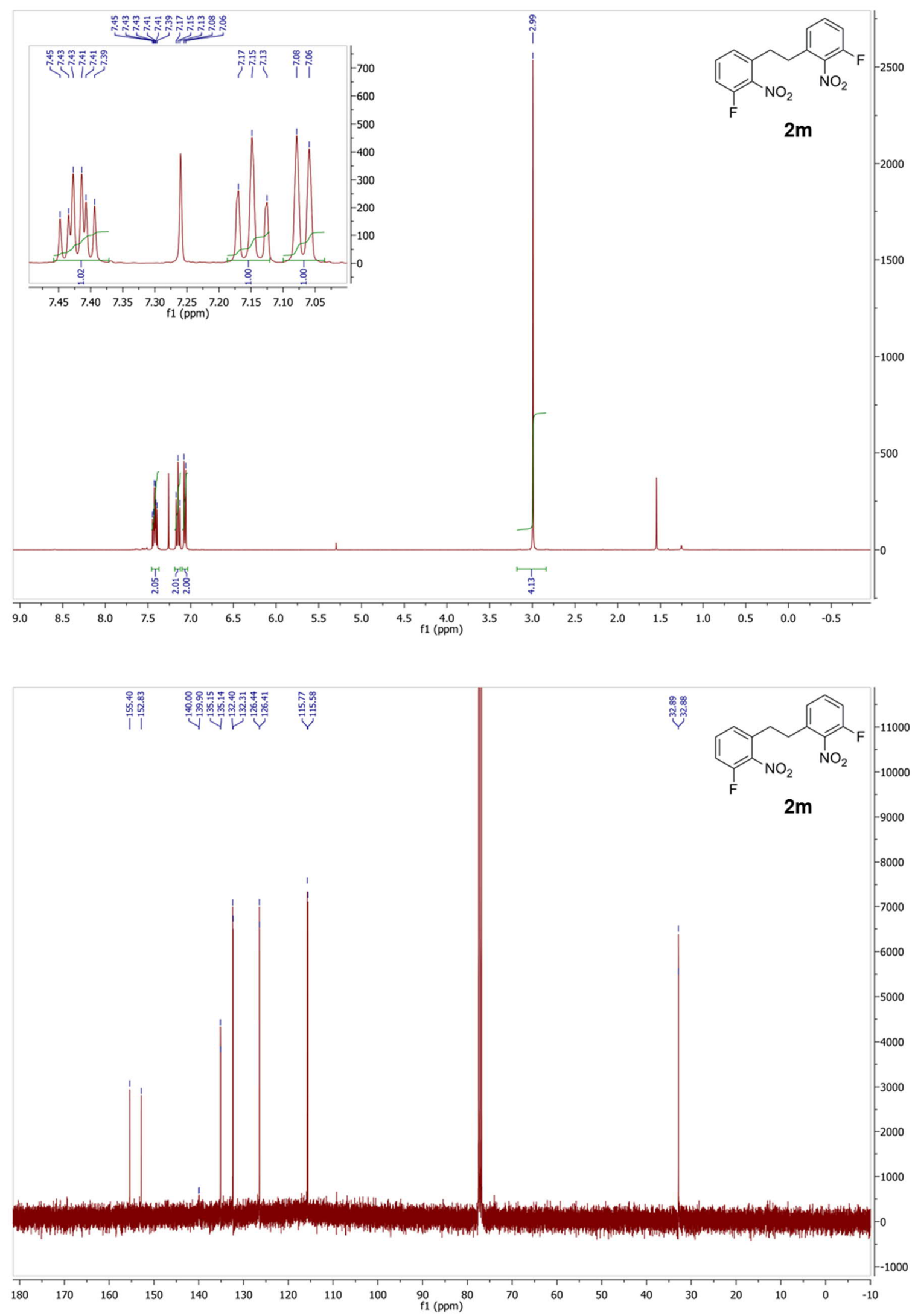

Figure S49. ${ }^{1} \mathrm{H}$ NMR $(400 \mathrm{MHz})$ and ${ }^{13} \mathrm{C}$ NMR (101 MHz) spectra of 1,2-bis(3-fluoro-2nitrophenyl)ethane $\mathbf{2 m}$ in $\mathrm{CDCl}_{3}$. 


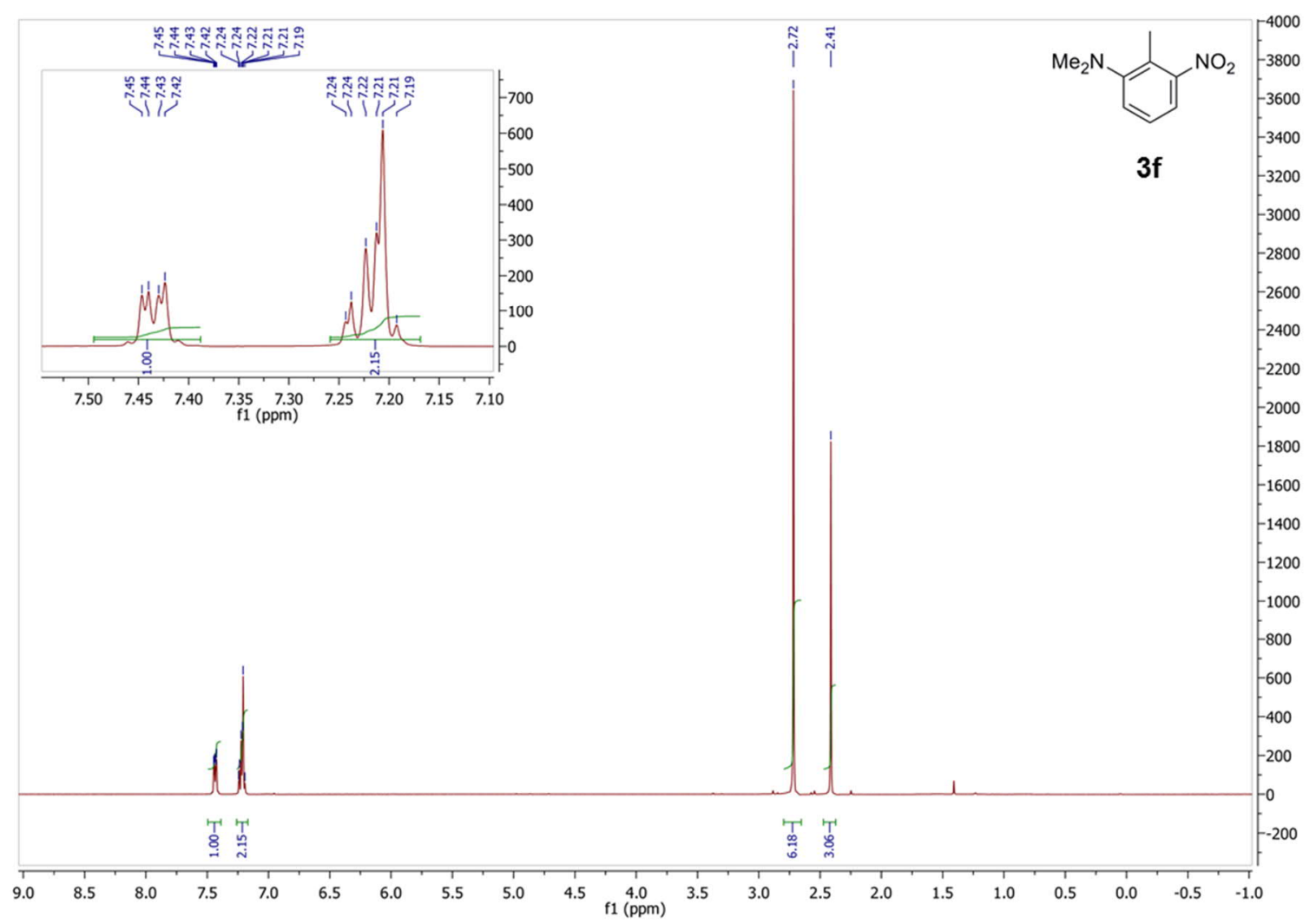

Figure S50. ${ }^{1} \mathrm{H}$ NMR (400 MHz) spectrum of 6-dimethylamino-2-nitrotoluene $3 \mathbf{f}$ in $\mathrm{CDCl}_{3}$. 

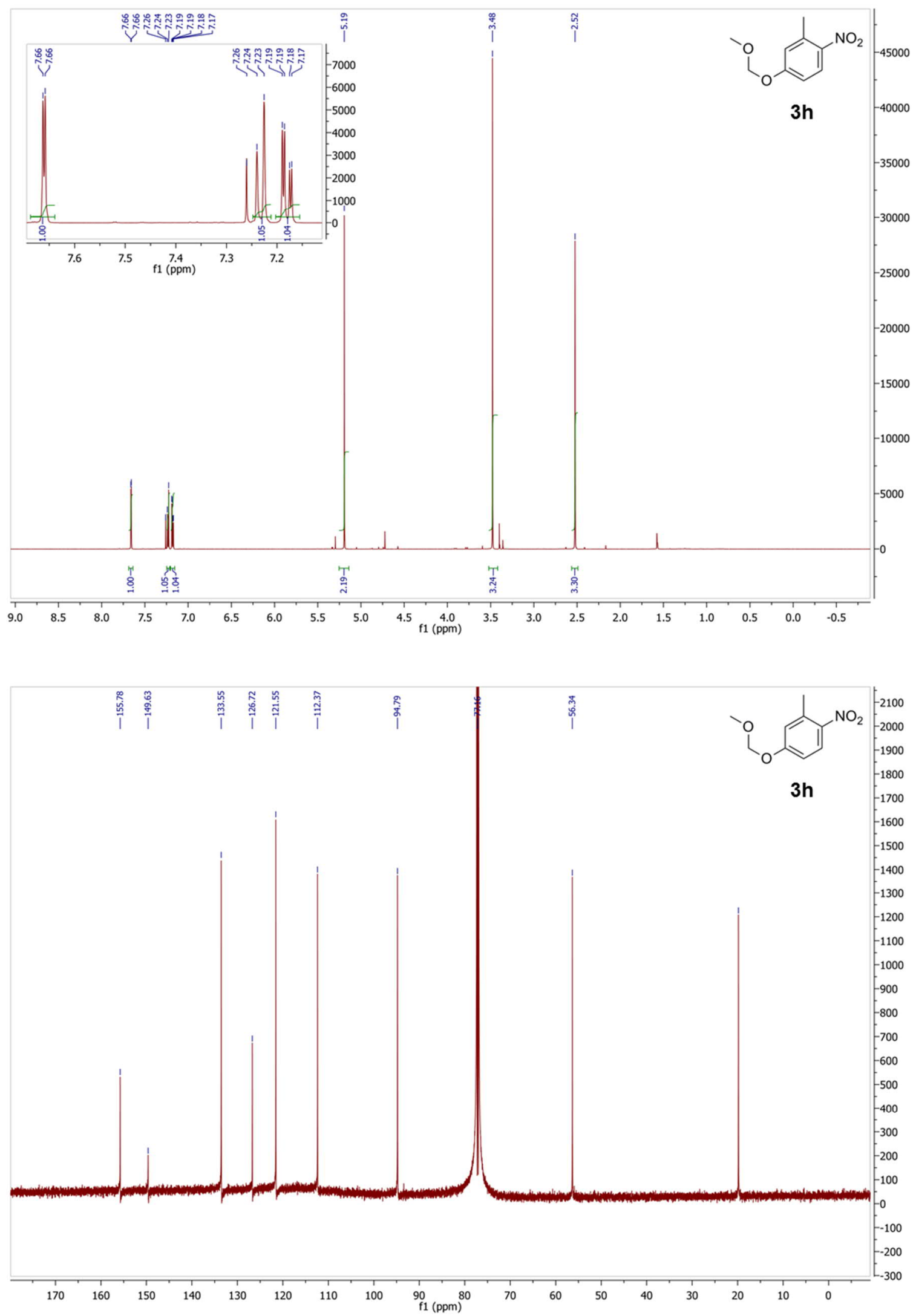

Figure S51. ${ }^{1} \mathrm{H}$ NMR (400 MHz) and ${ }^{13} \mathrm{C}$ NMR (101 MHz) spectra of 5-(methoxymethoxy)-2nitrotoluene $\mathbf{3 h}$ in $\mathrm{CDCl}_{3}$. 

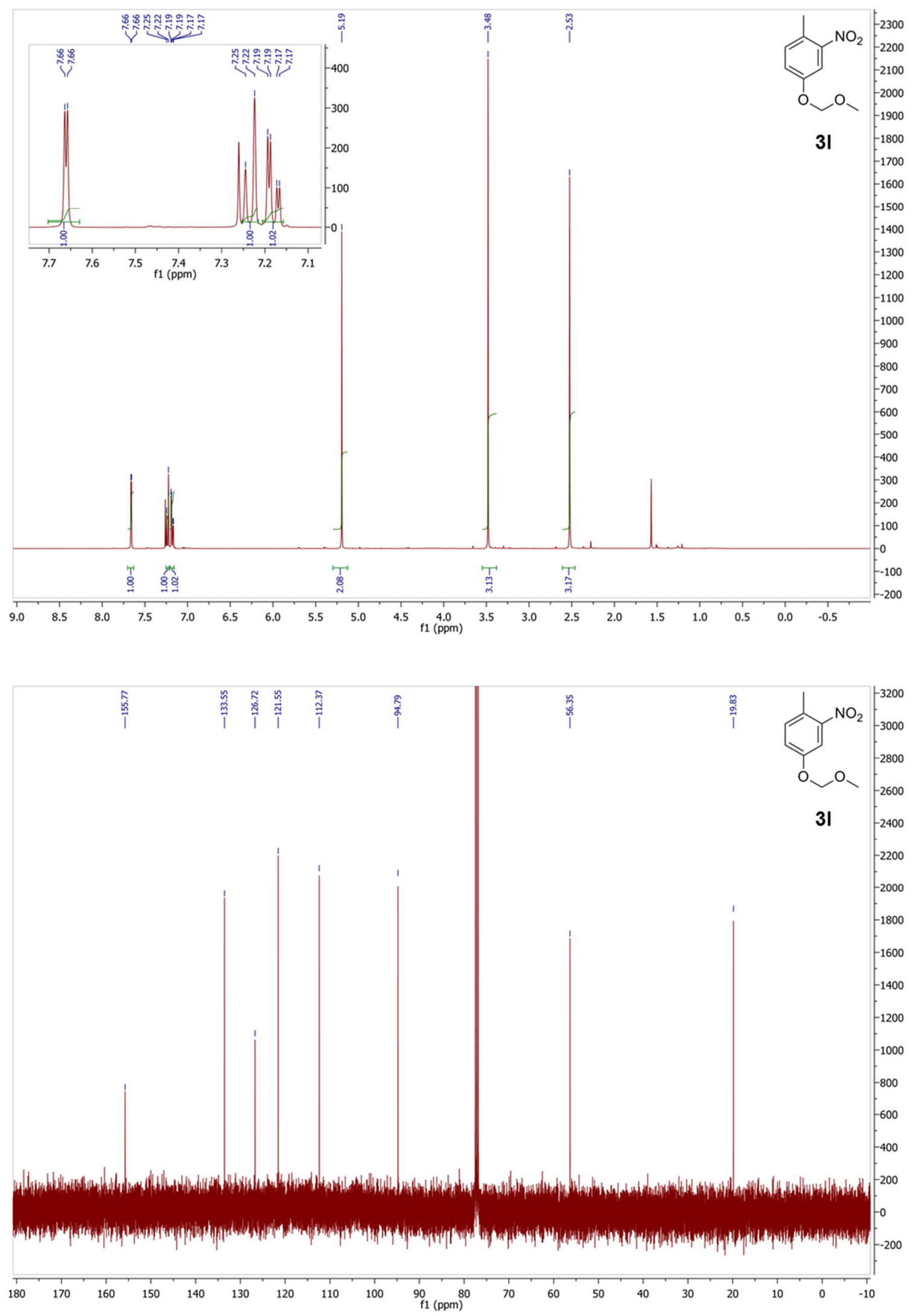

Figure S52. ${ }^{1} \mathrm{H}$ NMR (400 MHz) and ${ }^{13} \mathrm{C}$ NMR (101 MHz) spectra of 4-(methoxymethoxy)-2nitrotoluene $3 \mathbf{l}$ in $\mathrm{CDCl}_{3}$. 